\title{
The Japanese Clinical Practice Guideline for acute kidney injury 2016
}

\author{
Kent Doi ${ }^{1}$, Osamu Nishida ${ }^{2}$, Takashi Shigematsu ${ }^{3}$, Tomohito Sadahiro ${ }^{4}$, Noritomo Itami ${ }^{5}$, Kunitoshi Iseki ${ }^{6}$, \\ Yukio Yuzawa ${ }^{7}$, Hirokazu Okada ${ }^{8}$, Daisuke Koya ${ }^{9}$, Hideyasu Kiyomoto ${ }^{10}$, Yugo Shibagaki ${ }^{11}$, Kenichi Matsuda ${ }^{12}$, \\ Akihiko Kato ${ }^{13}$, Terumasa Hayashi ${ }^{14}$, Tomonari Ogawa ${ }^{15}$, Tatsuo Tsukamoto ${ }^{16}$, Eisei Noiri ${ }^{17}$, Shigeo Negi ${ }^{3}$, \\ Koichi Kamei $^{18}$, Hirotsugu Kitayama ${ }^{19}$, Naoki Kashihara ${ }^{20}$, Toshiki Moriyama ${ }^{21}$, Yoshio Terada ${ }^{22^{*}}$ and The Japanese \\ Clinical Practice Guideline for Acute Kidney Injury 2016 Committee
}

\begin{abstract}
Acute kidney injury (AKI) is a syndrome which has a broad range of etiologic factors depending on different clinical settings. Because AKI has significant impacts on prognosis in any clinical settings, early detection and intervention are necessary to improve the outcomes of AKI patients. This clinical guideline for AKI was developed by a multidisciplinary approach with nephrology, intensive care medicine, blood purification, and pediatrics. Of note, clinical practice for AKI management which was widely performed in Japan was also evaluated with comprehensive literature search.
\end{abstract}

Keywords: Acute kidney injury, Atrial natriuretic peptide, Biomarker, Blood purification, Long-term follow-up, Nafamostat mesilate

\section{CQ1: What is the concept of AKI, and what are the key elements of its clinical practice?}

Recommendation: AKI is a syndrome associated with a broad spectrum of diseases and a variety of underlying pathologies. Therefore, differentiation of the causes and elimination of the reversible factors are always required.

Strength of recommendation: Not graded

Quality of evidence: D

\section{Commentary}

In the past, the pathology associated with sudden renal impairment was characterized as an acute renal failure (ARF). However, in the 2000s, the joint efforts of specialists in

\footnotetext{
* Correspondence: terada@kochi-u.ac.jp

In 2016, the Japanese Society of Nephrology, the Japanese Society of Intensive Care Medicine, the Japanese Society of Dialysis Therapy, the Japanese Society of Blood Purification in Critical Care, and the Japanese Society of Pediatric Nephrology established the Committee of the Japanese Clinical Practice Guideline for Acute Kidney Injury 2016, which published in Jpn J Nephrol 2017, 59(4): 419-533. This is the English version of that report. The original work is at "https://cdn.jsn.or.jp/guideline/pdf/419-533.pdf."This article has been co-published in Clinical and Experimental Nephrology and Renal Replacement Therapy.

${ }^{22}$ Department of Endocrinology, Metabolism and Nephrology, Kochi Medical School, Kochi University, Kohasu, Oko-cho, Nankoku 783-8505, Japan Full list of author information is available at the end of the article
}

fields including nephrology, intensive care medicine, and cardiovascular medicine led to the introduction of a novel concept called acute kidney injury (AKI). Although both ARF and AKI designate clinical conditions that present with sudden renal impairment and renal tissue damage, their respective clinical backgrounds leading to onset are thought to differ. In cases of ARF, high invasiveness is assumed to lead to sudden renal impairment in patients with relatively few comorbidities. In addition, as ARF is thought to be essentially a reversible disease, there was little awareness of its poor outcomes; greater attention was paid to the differentiation of the causes and to the countermeasures against the complications associated with renal failure than to the need for early detection. However, as medical care progressed, patients such as high-risk elderly subjects who were not deemed to be candidates for invasive therapy came to be treated in intensive care units (ICUs). Eventually, there grew to be widespread awareness of the increase in cases of sudden kidney injury comorbid with sepsis and multiple organ failure and of the incredibly poor outcomes in these cases. This led to kidney injury as a subset of multiple organ failure to be reconsidered as AKI in intensive care medicine. Thus, AKI was proposed as a novel 
disease concept to emphasize early diagnosis and early intervention for the improvement of prognoses.

Meanwhile, the RIFLE [1], AKIN [2], and KDIGO [3] diagnostic criteria were introduced in an effort to establish unified international diagnostic criteria. The present guideline recommends the use of the KDIGO diagnostic criteria (see the "CQ2-1: Should the diagnosis of AKI be based on the KDIGO diagnostic criteria?" section). However, these criteria are based solely on the serum creatinine ( $\mathrm{sCr}$ ) and urine output; they do not take into account the cause or site of the kidney injury or the location and mode of onset of AKI. Thus, as AKI refers to a syndrome with a broad spectrum of diseases and a variety of pathophysiologies, it calls for constant differentiation of the causes and elimination of the reversible factors. The KDIGO Clinical Practice Guideline for AKI [3] also recommends searching for and assessing the cause of the syndrome whenever possible, particularly in regard to reversible causes (recommendations 2.1.3 and 2.3.1).

\section{CQ2-1: Should the diagnosis of AKI be based on the KDIGO diagnostic criteria?}

Recommendation: The KDIGO criteria are superior to the RIFLE criteria and to the AKIN criteria in predicting survival outcomes; therefore, we suggest using the KDIGO criteria to diagnose AKI. However, it is unknown which criteria should be used to predict the renal outcomes.

Strength of recommendation: 2

Quality of evidence: C

\section{Summary of evidence}

We identified 11 observational studies that compared the KDIGO with the AKIN and RIFLE criteria and that assessed death as an outcome. However, they did not assess the initiation of dialysis. In these 11 observational studies, the comparisons of the AKI diagnosis based on the KDIGO criteria versus those based on the RIFLE and AKIN criteria showed that the KDIGO criteria are more precise than, or as precise as, the RIFLE and AKIN criteria in reflecting the in-hospital mortality.

\section{Commentary}

In the past, acute renal failure (ARF) was diagnosed and classified according to several different criteria. In response to the growing call for unified international diagnostic criteria, the Acute Dialysis Quality Initiative (ADQI) published the RIFLE (Risk, Injury, Failure, Loss, End-stage kidney disease) criteria in 2004 (Table 1) [1, 4]. The RIFLE criteria distinguished three degrees of severity (risk, injury, and failure), with the latter defined as an increase in the serum creatinine $(\mathrm{sCr})$, a decline in the glomerular filtration rate (GFR), and a reduction in the urine output, and two types of clinical outcomes (loss and end-stage kidney disease). In 2004, the members of the International Society of Nephrology, the American Society of Nephrology, the National Kidney Foundation (in the USA), and the European Society of Intensive Care Medicine founded the Acute Kidney Injury Network (AKIN); as a replacement for the term ARF, the AKIN advocated the concept of acute kidney injury (AKI), which encompasses earlier stages of kidney injury. On the other hand, after the RIFLE criteria were published, a mere $0.3 \mathrm{mg} / \mathrm{dL}$ increase in $\mathrm{sCr}$ was reported to affect the survival prognosis and the clinical course of AKI $[5,6]$.

In 2007, the AKIN proposed the AKIN criteria, which were a revision of the RIFLE criteria (Table 2) [2]. The AKIN criteria included milder increases in $\mathrm{sCr}(0.3 \mathrm{mg} /$ $\mathrm{dL}$ ) and added the time course of the $\mathrm{sCr}$ increase (within $48 \mathrm{~h}$ ) to the diagnostic criteria. By contrast, a reduced GFR was removed from the RIFLE criteria. In addition, while both the AKIN criteria and the RIFLE criteria included the urine output, the AKIN criteria specified that when making a diagnosis based on the urine output alone, urinary tract obstructions and easily reversible causes of a reduced urine output were to be excluded and an adjustment was to be made for the body fluid volume. In addition, the RIFLE criteria's loss and end-stage kidney disease were judged to be the outcomes of AKI and were removed from the AKIN criteria's staging system. Furthermore, patients who had started renal replacement therapy (RRT) became classified as stage 3 regardless of their $\mathrm{sCr}$ and urine output prior to the RRT initiation.

Table 1 RIFLE criteria

\begin{tabular}{lll}
\hline & GFR criteria & Urine output criteria \\
\hline Risk & Increase in $\mathrm{SCr} \geq 1.5 \times$ baseline or decrease in $\mathrm{GFR} \geq 25 \%$ & $\cup \mathrm{UO}<0.5 \mathrm{~mL} / \mathrm{kg} / \mathrm{h} \times 6 \mathrm{~h}$ \\
Injury & Increase in $\mathrm{s} C r \geq 2.0 \times$ baseline or decrease in $\mathrm{GFR} \geq 50 \%$ & $\cup O<0.5 \mathrm{~mL} / \mathrm{kg} / \mathrm{h} \times 12 \mathrm{~h}$ \\
Failure & Increase in $\mathrm{s} C r \geq 3.0 \times$ baseline or an absolute $\mathrm{s} C r \geq 4.0 \mathrm{mg} / \mathrm{dL}$ & $\cup O<0.3 \mathrm{~mL} / \mathrm{kg} / \mathrm{h} \times 24 \mathrm{~h}$ or anuria $\times 12 \mathrm{~h}$ \\
& with an acute rise of at least $0.5 \mathrm{mg} / \mathrm{dL}$ or decrease in $\mathrm{GFR} \geq 75 \%$ & \\
Loss & Complete loss of kidney function $>4$ weeks & \\
ESKD & End-stage renal disease (dialysis dependent $>3$ months) & \\
\hline
\end{tabular}

GFR glomerular filtration rate, $s C r$ serum creatinine, ESKD end-stage kidney disease, $U O$ urine output 
Table 2 AKIN criteria

\begin{tabular}{|c|c|c|}
\hline \multirow[t]{4}{*}{ Definition } & \multicolumn{2}{|l|}{ 1. Increased in $\mathrm{SCr}$ of $\geq 0.3 \mathrm{mg} / \mathrm{dL}(48 \mathrm{~h})$} \\
\hline & \multicolumn{2}{|l|}{ 2. $\mathrm{s} C r$ changes $\geq 1.5 \times$ baseline ( $48 \mathrm{~h}$ ) } \\
\hline & \multicolumn{2}{|l|}{ 3. $\cup O<0.5 \mathrm{~mL} / \mathrm{kg} / \mathrm{h} \times 6 \mathrm{~h}$} \\
\hline & sCr criteria & UO criteria \\
\hline Stage 1 & Increased in $\mathrm{s} C r$ of $\geq 0.3 \mathrm{mg} / \mathrm{dL}$ or increase to $1.5-2.0 \times$ baseline & $\cup O<0.5 \mathrm{~mL} / \mathrm{kg} / \mathrm{h} \times 6 \mathrm{~h}$ \\
\hline Stage 2 & Increase in $\mathrm{s} C r$ to $2.0-3.0 \times$ baseline & $\mathrm{UO}<0.5 \mathrm{~mL} / \mathrm{kg} / \mathrm{h} \times 12 \mathrm{~h}$ \\
\hline Stage 3 & $\begin{array}{l}\text { Increase in } \mathrm{s} C r>3.0 \times \text { baseline or } \mathrm{s} C r \geq 4.0 \mathrm{mg} / \mathrm{dL} \text { with an } \\
\text { acute rise of at least } 0.5 \mathrm{mg} / \mathrm{dL} \text { or Initiation of RRT }\end{array}$ & $\mathrm{UO}<0.3 \mathrm{~mL} / \mathrm{kg} / \mathrm{h} \times 24 \mathrm{~h}$ or anuria $\times 12 \mathrm{~h}$ \\
\hline
\end{tabular}

$s C r$ serum creatinine, $U O$ urine output, $R R T$ renal replacement therapy

In 2012, the Kidney Disease: Improving Global Outcomes (KDIGO) group assembled all the available evidence into their own clinical practice guideline for AKI and proposed the KDIGO criteria, which integrate the RIFLE and AKIN criteria (Table 3) [3]. The KDIGO criteria diverge from the AKIN criteria in that the time course for a 1.5-fold increase in $\mathrm{sCr}$ from baseline was changed from within $48 \mathrm{~h}$ to within 7 days. Thus, as the KDIGO criteria encompass more gradual increases in $\mathrm{sCr}$, they have made the number of patients diagnosed with AKI likely to increase.

As described above, three sets of diagnostic criteria for AKI have been proposed: the RIFLE, AKIN, and KDIGO criteria. The utility of the KDIGO criteria, the most recent of the three sets, has been compared with that of the two older sets. In a prospective, multicenter observational study of 3107 intensive care unit (ICU) patients, Luo et al. reported the percentages of patients diagnosed with AKI according to the RIFLE, AKIN, and KDIGO criteria using both the $\mathrm{sCr}$ and urine output and compared their in-hospital mortality rates [7]. The percentages of patients diagnosed with AKI according to the RIFLE, AKIN, and KDIGO criteria were 46.9, 38.4, and $51.0 \%$, respectively; thus, the number of patients diagnosed with AKI was significantly higher when using the KDIGO criteria than when using either the AKIN or RIFLE criteria. The patients diagnosed with AKI based on the KDIGO criteria had poorer survival outcomes than those diagnosed using the AKIN criteria, although there was no significant difference in the survival outcomes of patients diagnosed using the RIFLE criteria. In a retrospective multicenter study of 1005 adult patients hospitalized for acute heart failure, Li et al. compared the percentages of patients diagnosed with AKI within 7 days of hospitalization using the KDIGO, AKIN, and RIFLE criteria, as well as the patients' in-hospital mortality rates [8]. Using only the $\mathrm{sCr}$ criterion, the percentages of patients diagnosed with AKI according to the KDIGO, AKIN, and RIFLE criteria were 38.9, 34.7, and 32.1\%, respectively. A total of 110 patients (10.9\%) were diagnosed with AKI with the KDIGO criteria but not with the RIFLE or AKIN criteria. A total of 18.4\% of the patients who died in the hospital were diagnosed with AKI according to the KDIGO criteria only; this group was at a high risk of in-hospital death. In a study of 1050 patients hospitalized for acute myocardial infarction, Rodrigues et al. compared the percentages of patients diagnosed with AKI according to the RIFLE and KDIGO criteria using the $\mathrm{sCr}$ criterion only, as well as their mortality rates [9]. A total of $14.8 \%$ of patients were diagnosed with AKI with the RIFLE criteria versus $36.6 \%$ with the KDIGO criteria. In comparison with patients without AKI, the 30-day and 1-year mortality hazard ratios for patients diagnosed with AKI according to the KDIGO criteria but not to the RIFLE criteria were 2.55 and 2.28 , respectively.

In other studies of the AKI diagnostic criteria in hospitalized patients [10, 11], ICU patients [12-14], acute decompensated heart failure [15], patients after cardiac surgery [16], and sepsis [17], the KDIGO criteria were reported to be equal or superior to the RIFLE and AKIN criteria in their ability to predict the survival outcomes. Based on the above, the KDIGO criteria are considered to be more useful in their survival outcome prediction ability than the RIFLE or AKIN criteria for the diagnosis of AKI.

Table 3 KDIGO criteria

\begin{tabular}{|c|c|c|}
\hline \multirow[t]{2}{*}{ Definition } & $\begin{array}{l}\text { 1. Increased in } \mathrm{SCr} \text { of } \geq 0.3 \mathrm{mg} / \mathrm{dL}(48 \mathrm{~h}) \\
\text { 2. } \mathrm{s} C r \text { changes } \geq 1.5 \times \text { baseline ( } 7 \text { days) } \\
\text { 3. } \mathrm{UO}<0.5 \mathrm{~mL} / \mathrm{kg} / \mathrm{h} \times 6 \mathrm{~h}\end{array}$ & \\
\hline & sCr criteria & UO criteria \\
\hline Stage 1 & Increased in $\mathrm{s} C r$ of $\geq 0.3 \mathrm{mg} / \mathrm{dL}$ or increase to $1.5-1.9 \times$ baseline & $\mathrm{UO}<0.5 \mathrm{~mL} / \mathrm{kg} / \mathrm{h} \times 6 \mathrm{~h}$ \\
\hline Stage 2 & Increase in sCr to $2.0-2.9 \times$ baseline & $\mathrm{UO}<0.5 \mathrm{~mL} / \mathrm{kg} / \mathrm{h} \times 12 \mathrm{~h}$ \\
\hline Stage 3 & Increase in $\mathrm{s} C r>3.0 \times$ baseline or $\mathrm{s} C r \geq 4.0 \mathrm{mg} / \mathrm{dL}$ or Initiation of RRT & $\mathrm{UO}<0.3 \mathrm{~mL} / \mathrm{kg} / \mathrm{h} \times 24 \mathrm{~h}$ or anuria $\times 12 \mathrm{~h}$ \\
\hline
\end{tabular}

$s C r$ serum creatinine, $U O$ urine output, $R R T$ renal replacement therapy 


\section{Literature review}

PubMed was searched for relevant studies published between January 1990 and July 2015, and papers related to the present $C Q$ were identified from the search results.

\section{CQ2-2: When diagnosing AKI, how should an unknown baseline renal function be estimated?}

Recommendation: Whenever possible, the baseline renal function should be determined using multiple methods, and the potential presence of chronic kidney disease (CKD) and other comorbidities should be assessed.

\section{Strength of recommendation: 2}

Quality of evidence: C

\section{Summary of evidence}

Several methods have been suggested to estimate the baseline renal function. However, compared to the use of the known baseline function, all of these methods have been reported to yield a certain rate of false positives or false negatives in their AKI diagnoses and mortality predictions.

\section{Commentary}

The diagnosis of acute kidney injury (AKI) requires the baseline renal function; however, in actual clinical practice, the patient's history of examination and his/her baseline renal function are often unknown. These cases require an estimation of the baseline renal function, and many methods have been proposed (Table 4).

To compare the estimated baseline renal function with the known baseline renal function, we identified seven observational studies that used the AKI diagnosis as an outcome [18-24] and two observational studies that used the all-cause mortality as an outcome [20, 22]. While two of the seven studies included all hospitalized patients $[19,22]$, two of them limited the subjects to intensive care unit (ICU) patients [23, 24], two used only patients undergoing cardiac surgery [20, 21], and one study only included patients with cirrhosis [18]. All these studies assumed the lower limit of the normal renal function to be an estimated glomerular filtration rate (eGFR) of $75 \mathrm{~mL} / \mathrm{min} / 1.73 \mathrm{~m}^{2}$ (as suggested by the KDIGO Clinical Practice Guideline [3]) and examined a way to back-calculate the serum creatinine $(\mathrm{sCr})$ based on the MDRD equation. In the six studies whose subjects included all hospitalized patients, the ICU patients only, and the cardiac surgery patients only, an assumed baseline renal function of eGFR $75 \mathrm{~mL} / \mathrm{min} / 1.73 \mathrm{~m}^{2}$ yielded false-positive AKI diagnoses. Four of these studies $[19,21,23,24]$ stated that false positives were especially frequent in patients with a known eGFR $<60 \mathrm{~mL} /$ $\min / 1.73 \mathrm{~m}^{2}$. On the contrary, in the study whose subjects included cirrhosis patients only, an assumed baseline renal function of eGFR $75 \mathrm{~mL} / \mathrm{min} / 1.73 \mathrm{~m}^{2}$ yielded false-negative AKI diagnoses. In a study that was not taken into account due to its unsuitable outcome, Zavada et al. indicated that the estimated $\mathrm{sCr}$ was higher than the known $\mathrm{sCr}$ in young people [25]. The two observational studies that used the all-cause mortality as an outcome reported that the mortality rates were reduced by $\mathrm{sCr}$ estimation methods that frequently yielded false-positive AKI diagnoses, while the mortality rates were increased by estimation methods that frequently yielded false-negative diagnoses.

In conclusion, there is currently no specific baseline $\mathrm{sCr}$ estimation method on par with a measured baseline $\mathrm{sCr}$. The easy method that involves the calculation of the $\mathrm{sCr}$ based on an eGFR of $75 \mathrm{~mL} / \mathrm{min} / 1.73 \mathrm{~m}^{2}$ is tolerable; however, this method often overestimates the $\mathrm{sCr}$ in young people and cirrhosis patients and underestimates it in chronic kidney disease (CKD) patients. Therefore, we suggest that whenever possible, the baseline renal function should be determined using multiple methods while also confirming whether the CKD and other comorbidities are present based on methods such as image searches to check for renal atrophy.

Table 4 Estimation of unknown baseline serum creatinine

\begin{tabular}{|c|c|c|}
\hline Estimating baseline $\mathrm{s} C \mathrm{r}$ method & Characteristics in the diagnosis of AKI & Reference \\
\hline $\begin{array}{l}\text { An estimated sCr determined by back-calculation using MDRD assuming a } \\
\text { GFR of } 75 \mathrm{~mL} / \mathrm{min} / 1.73 \mathrm{~m}^{2}\end{array}$ & Low specificity especially in CKD patients & [18-24] \\
\hline $\begin{array}{l}\text { An estimated } \mathrm{s} C \mathrm{r} \text { determined by back-calculation using MDRD assuming } \\
\text { a GFR of } 100 \mathrm{~mL} / \mathrm{min} / 1.73 \mathrm{~m}^{2}\end{array}$ & Very high sensitivity and very low specificity & [23] \\
\hline The first admission $\mathrm{sCr}$ & Low sensitivity & [22] \\
\hline A minimum inpatient sCr during the first 7 days & Low specificity & [22] \\
\hline A minimum $\mathrm{s} C r$ during the first 7 days in the ICU & $\begin{array}{l}\text { Low specificity although tendency to underestimate the AKI } \\
\text { stage }\end{array}$ & [23] \\
\hline $\begin{array}{l}\text { An estimated sCr using multiple imputation methods such as sex, race, } \\
\text { comorbidity (CKD, etc.), and a minimum inpatient } \mathrm{s} C r\end{array}$ & High specificity & [19] \\
\hline A minimum inpatient $\mathrm{s} C r$ & & [11] \\
\hline $\mathrm{sCr}=1.0 \mathrm{mg} / \mathrm{dL}$ (male) $/ 0.8 \mathrm{mg} / \mathrm{dL}$ (female) & & [25] \\
\hline
\end{tabular}




\section{Literature review}

PubMed was searched for relevant studies published up to July 2015, and papers related to the present CQ were identified from the search results.

\section{CQ2-3: Should the AKI staging with the urine output be included in addition to the serum creatinine for the predictions of the AKI outcomes?}

Recommendation: In the RIFLE, AKIN, and KDIGO criteria, the inclusion of the urine output along with the serum creatinine to determine the AKI stage yields more accurate reflections of the survival outcomes and the renal outcomes than the determination of the AKI stage based on the serum creatinine alone. Therefore, we suggest that the AKI staging should involve the urine output whenever possible.

Strength of recommendation: 2

Quality of evidence: B

\section{Summary of evidence}

We identified seven observational studies that used death as an outcome. In the studies of ICU patients, the inclusion of the urine output as a criterion significantly improved the survival outcome predictions. In one of these studies, the renal outcome prediction was also improved; however, a study of patients after cardiac surgery indicated a potential for overdiagnosis. Because no study of outpatients or general ward patients has been conducted, it is unclear whether the above results can be generalized.

\section{Commentary}

During the 10 years since the concept of AKI was introduced, three sets of diagnostic criteria/classifications have been proposed: the RIFLE, AKIN, and KDIGO. All these criteria sets enable the diagnosis and staging of AKI based on changes in the serum creatinine $(\mathrm{sCr})$ or the urine output [1-3]. In many previous clinical studies, AKI was diagnosed and staged according to the $\mathrm{sCr}$ alone, and a slight increase in the $\mathrm{sCr}$ was reported to affect the survival outcomes $[3,6]$. However, few clinical studies have used the urine output as a criterion for the diagnosis and staging of AKI. Therefore, we examined whether the urine output reflects the survival outcomes of AKI as accurately as the $\mathrm{sCr}$ and whether the inclusion of the urine output in the determination of the AKI stage reflects the survival outcomes more accurately than a determination based on the $\mathrm{sCr}$ alone.

To compare the $\mathrm{sCr}$ and the urine output, we adopted seven observational studies that used death as an outcome [26-32]. All these studies were conducted in intensive care units (ICUs); none of them involved outpatients or patients in general wards. Regarding the
AKI diagnostic criteria, three studies used the RIFLE criteria [26, 27, 30], two used the AKIN criteria [31, 32], and two used the KDIGO criteria [28, 29]. In six of these studies, the inclusion of the urine output with the $\mathrm{sCr}$ in the AKI diagnosis significantly improved the survival outcome predictions [27-32]; furthermore, in one of these six studies, the renal outcome prediction ability was also improved [27].

In an analysis of 155,624 patients hospitalized in ICUs on an emergency admission, Harris et al. reported that the urine output was a more powerful predictor of the survival outcomes than the $\mathrm{sCr}$ [26]. In a study of 32,045 adult ICU patients classified according to the KDIGO $\mathrm{sCr}$ and urine output criteria, Kellum et al. demonstrated that patients who fulfilled both the $\mathrm{sCr}$ and urine output criteria were at the highest risk of death and the initiation of permanent renal replacement therapy (RRT), while isolated oliguria was associated with a long-term risk of death even when the $\mathrm{sCr}$ criterion was not fulfilled [27]. Similarly, in an analysis of 390 septic shock patients, Leedahl et al. reported that persistent oliguria was a risk factor for death by day 28 [28]. In a study of 260 ICU patients, Wlodzimirow et al. compared the combined use of the RIFLE's $\mathrm{sCr}$ and urine output criteria (RIFLEsCr+urine output) with the use of the $\mathrm{sCr}$ criterion alone (RIFLEsCr); they reported that the RIFLEsCr was associated with a delayed AKI diagnosis and higher mortality [29]. Furthermore, Han et al. [30] and Macedo et al. [31] also reported that the addition of the urine output criterion enabled a more accurate AKI diagnosis than the use of the $\mathrm{sCr}$ criterion alone. $\mathrm{Al}$ though some studies have featured different urine output criterion values, overall, the assessment of the urine output has been shown to improve the accuracy of the AKI diagnosis. However, in a comparison of the $\mathrm{sCr}$ criterion alone with the urine output criterion alone for the diagnosis of AKI in patients after cardiac surgery, Lagny et al. indicated that the use of the urine output criterion alone could lead to overdiagnosis [32]. In a recent multicenter prospective study that assessed the association between the hourly urine output and mortality, Vaara et al. reported that patients who fulfilled both the $\mathrm{sCr}$ and urine output criteria had the highest rate of RRT initiation and the highest 90-day mortality, while isolated oliguria was associated with poor outcomes; these results affirm the importance of measuring the hourly urine output and the need to combine the urine output criterion with the $\mathrm{sCr}$ criterion [33]. Moreover, in using the urine output criterion in the diagnosis and staging of $\mathrm{AKI}$, there is a concern that the use of diuretics may change the urine output, causing underestimation of the AKI severity. However, in their analysis of the effects of diuretics on the AKI diagnosis, Han et al. reported that the inclusion of the urine output criterion alongside the 
$\mathrm{sCr}$ criterion played an additional role in the diagnosis and staging of AKI regardless of whether diuretics were used [30].

To summarize the above studies, the inclusion of the urine output along with the $\mathrm{sCr}$ to determine the AKI stage improves the sensitivity of the AKI diagnosis and yields more accurate reflections of the survival and renal outcomes than AKI staging based on the $\mathrm{sCr}$ alone. Therefore, we suggest that AKI staging should involve the urine output whenever possible.

\section{Literature review}

PubMed was searched for relevant studies published between January 1990 and August 2015, and papers related to the present CQ were identified from the search results.

\section{CQ3-1: What should be assessed as risk factors for AKI development in cardiac surgery?}

Recommendation: We suggest that factors such as age, preoperative renal dysfunction, and the duration of the cardiopulmonary bypass should be assessed as risk factors.

Strength of recommendation: 2

Quality of evidence: C

\section{Summary of evidence}

We identified seven papers that assessed the risk of development of AKI in cardiac surgery. All of them were observational studies. Certain observational studies have stated that transcatheter aortic valve replacement (TAVR) and transcatheter aortic valve implantation (TAVI), which have become more common with the recent aging of society, do not match the same risk of AKI observed in cardiac surgery.

\section{Commentary}

\section{Background}

Acute kidney injury (AKI) is a comorbidity that complicates the perioperative management of body fluid; the risk of AKI development is reported to be particularly high in cardiac surgery [34]. In $\mathrm{Hu}$ et al.'s meta-analysis of 91 studies of cardiac surgery, the incidence of postoperative AKI was $22.3 \%$, while $2.3 \%$ of the patients required renal replacement therapy (RRT). Furthermore, the in-hospital mortality of patients who developed AKI following cardiac surgery was $10.7 \%$, and the mortality in long-term observation (1-5 years) was $30.0 \%$ [35]. Therefore, assessment of the risk of AKI development is crucial for patients scheduled to undergo cardiac surgery. Nearly all the relevant studies have been observational studies, which make them insufficient to demonstrate strong evidence; nevertheless, they have identified several potential risk factors (Table 5).

\section{Aging}

The aging of patients who undergo cardiac surgery may make their perioperative management more difficult. In a prospective observational study, Ozkayanak et al. reported that the risk of development of AKI from cardiac surgery increased with age (odds ratio 1.022, 95\% confidence interval 1.005-1.039) [36]. Nearly identical results have been demonstrated in retrospective observational studies [37, 38]. Regarding coronary artery bypass grafting (CABG), a prospective observational study limited to Asian patients showed that AKI developed significantly more frequently in patients aged $\geq 70$ years (odds ratio 1.350, 95\% confidence interval 1.085-1.679) [39]. A retrospective observational study of patients who had undergone cardiac surgery with a cardiopulmonary bypass also reported age as a significant risk factor for AKI development [40]. The risk of AKI development should be considered while treating elderly patients undergoing cardiac surgery.

\section{Preoperative renal impairment}

Preoperative renal dysfunction is known as a risk factor for perioperative AKI development. Observational studies of cardiac surgery patients have also reported that pre-AKI renal dysfunction was a potential risk factor for AKI development. Huang et al. reported that CKD stage G3 (odds ratio 1.68, 95\% confidence interval 1.12-2.52) and CKD stage G4 (odds ratio 3.01, 95\% confidence interval 1.57-6.03) were risk factors for AKI development after cardiac surgery [41]. In a prospective observational study of CABG patients, Guenancia et al. reported that a higher preoperative estimated glomerular filtration rate (eGFR) was associated with a lower risk of AKI development (odds ratio $0.97,95 \%$ confidence interval 0.96-0.99) [42]. In another prospective observational study of CABG patients, $\mathrm{Ng}$ et al. reported that a higher preoperative serum creatinine $(\mathrm{sCr})$ value was associated with an increased risk of AKI development (odds ratio 1.003, 95\% confidence interval 1.001-1.006) [39].

\section{Duration of cardiopulmonary bypass}

A cardiopulmonary bypass $(\mathrm{CPB})$ creates an extracorporeal environment and a non-physiological state in which a constant blood flow is maintained with a pump, independently from the heartbeat. In typical CPBs, the blood is diluted by $20-50 \%$ to reduce the hemoglobin concentration. During CPBs, the renal blood flow is affected by various factors, including hypothermia, blood dilution, hemolysis, microthrombi, and vasoactive drugs; these factors constrict the renal artery and reduce the renal blood flow. In a meta-analysis of nine studies on the correlation between the duration of CPBs in cardiac surgery and the development of $\mathrm{AKI}$, the $\mathrm{CPB}$ duration was reported to be 
Table 5 Risk factors for AKI development in cardiac surgery

\begin{tabular}{lllllllll}
\hline Reference & Author, year & Aging & Obesity & Diabetes & Hypertension & $\begin{array}{l}\text { Preoperative } \\
\text { anemia }\end{array}$ & $\begin{array}{l}\text { Preoperative renal } \\
\text { impairment }\end{array}$ & $\begin{array}{l}\text { Cardiopulmonary } \\
\text { bypass duration }\end{array}$ \\
\hline$[37]$ & Kristovic et al. 2015 & $\circ$ & $\circ$ & $\Delta$ & $\times$ & - & - & - \\
{$[38]$} & Joung et al. 2014 & $\circ$ & $\times$ & $\times$ & $\Delta$ & $\Delta$ & $\Delta$ & 0 \\
{$[39]$} & Ng et al. 2014 & $\circ$ & $\circ$ & $\Delta$ & $\circ$ & $\circ$ & 0 & - \\
{$[36]$} & Ozkaynak et al. 2014 & $\circ$ & $\circ$ & $\times$ & $\times$ & - & - & - \\
{$[43]$} & Kumar et al. 2012 & - & - & - & - & - & - \\
{$[40]$} & Parolari et al. 2012 & $\circ$ & - & - & - & - & - \\
{$[41]$} & Huang et al. 2011 & $\circ$ & - & $\circ$ & - & - & 0 \\
\hline
\end{tabular}

$\circ$ risk, $\Delta$ risk without significance, $\times$ not risk, - not evaluated

significantly associated with the development of AKI [43]. Off-pump surgery, which has become more common recently, could make the surgery less invasive for elderly heart disease patients in Japan. In a meta-analysis of randomized controlled trials (RCTs) involving CABG patients, Seabra et al. reported that compared to on-pump CABG, off-pump CABG significantly inhibited the postoperative AKI onset; however, no significant association was observed with the need for dialysis [44]. In an RCT that observed the long-term renal outcomes, the incidence of AKI development within 30 days after surgery was significantly lower after off-pump CABG (17.5\% vs $20.8 \%$, 95\% confidence interval: 0.72-0.97); however, at 1 year, there was no difference in the percentages of patients with a reduced eGFR. Therefore, recent RCTs have failed to sufficiently prove the efficacy of off-pump CABG for renoprotection.

\section{Other risk factors}

In addition to the risk factors stated above, observational studies have also assessed obesity, diabetes, hypertension, and anemia as potential risk factors; however, due to contradictory results, no conclusions have been reached [38-42]. Recently, transcatheter aortic valve implantation (TAVI) and transcatheter aortic valve replacement (TAVR) have become more common, since they can be performed with minimal invasiveness in the elderly and high-risk patients. In a meta-analysis of 13 studies, Elhmidi et al. reported that preoperative renal impairment was a significant risk factor for post-TAVI AKI development [45].

\section{Literature review}

PubMed was searched for relevant studies published between March 2011 and December 2015, and papers related to the present CQ were identified from the search results. The literature published before March 2011 was referenced from the KDIGO Clinical Practice Guideline for AKI.

\section{CQ3-2: What should be assessed as risk factors for AKI development in non-cardiac surgery?}

Recommendation: In liver transplantation, we suggest that the preoperative model for end-stage liver disease (MELD) score, the intraoperative blood transfusion volume, the intraoperative hypotension, and the use of vasopressors should be assessed as risk factors for AKI development. The potential risk factors related to other non-cardiac surgeries are unknown.

Liver transplantation:

Strength of recommendation: 2

Quality of evidence: $\mathrm{C}$

Other surgeries:

Strength of recommendation: Not graded

Quality of evidence: D

\section{Summary of evidence}

Among ten observational studies on the development of AKI following liver transplantation, five studies demonstrated a significant association between the development of AKI and the intraoperative blood transfusion volume. Two studies excluded chronic kidney disease (CKD), while two others found CKD to be a significant risk factor for AKI development. Two studies demonstrated that the MELD score and intraoperative hypotension or the use of vasopressors were associated with the development of AKI. Only three studies about lung transplantation and AKI development were found, and these studies did not demonstrate a consistent trend.

\section{Commentary Background}

The development of AKI is significantly associated with increased mortality. This lends great clinical significance to the development of AKI following non-cardiac surgery as well as cardiac surgery. Therefore, it is crucial to determine the incidence rate of AKI, the risk factors for its development, and its association with prognoses. Despite the existence of several studies about the development of AKI after liver transplantation, nearly all have 
been observational studies. Furthermore, there have been few studies on the development of AKI after non-cardiac surgeries other than liver transplantation.

\section{Liver transplantation}

In liver disease, the development of AKI is generally a risk factor for the progression of hepatic dysfunction and increased mortality [46]. In liver transplantation, one of the most invasive liver surgery procedures, postoperative AKI is associated with mortality; therefore, it is crucial to assess the risk factors that predict its development. Many studies have reported the incidence of AKI after liver transplantation; however, it has ranged greatly, between 17 and 95\% [47]. Recent investigations have primarily used the AKIN classification system; in retrospective studies published between 2013 and 2015, the incidence of the post-liver transplantation development of AKI ranged from 10 to 30\% [48-53]. In 2014, Leithead et al. reported an investigation of the AKI onset among 1152 patients who had undergone liver transplantation [48]. The study defined AKI as the progression to KDIGO stage 2 or higher within 1 week after transplantation. Based on this definition, the incidence of AKI was 33.8\%; factors such as the preoperative MELD score, preoperative hyponatremia, a preoperative $\mathrm{BMI} \geq 30 \mathrm{~kg} / \mathrm{m}^{2}$, intraoperative red blood cell transfusion, and a long warm ischemic time were identified as risk factors for AKI development [54]. In transplantation, the length of time from the stopping of the organ blood flow to the resumption of the blood flow following transplantation is defined as the ischemic time; the exposition of the organ to an ischemic state, particularly at a normal temperature, increases the likelihood that cells will die. This time is called the warm ischemic time; the ideal time is $0 \mathrm{~min}$ for the heart and liver and $30 \mathrm{~min}$ for the kidneys and lungs. In order to achieve these ideal times, the organs must be cooled at an early stage to reduce the cellular metabolism. The fact that these unique liver transplantation parameters are associated with AKI is fascinating in terms of organ crosstalks. There have been ten observational studies on the development of AKI following liver transplantation [48-53, 55-58]. Five of them have reported an intraoperative red blood cell transfusion as an independent risk factor for AKI development, while two studies have reported the preoperative MELD score, intraoperative hypertension, and the use of vasopressors as independent risk factors. A retrospective cohort study in 2015 reported the same results in relation to liver resection [59]. In that study, 78 of the 642 patients who had undergone liver resection developed AKI (as defined according to the AKIN classification) within
72 h. Preoperative renal impairment, preoperative hypertension, and intraoperative red blood cell transfusion were identified as risk factors for AKI development. However, this study is the only one to have examined the development of AKI after liver resection to date.

\section{Lung surgery}

There have been three studies on AKI following lung surgery, and all of them have been retrospective cohort studies [60-62]. In one of them, George et al. assessed the need for postoperative RRT in a multicenter study of 12,108 patients and found an AKI incidence of 5.5\%; increasing age, the male gender, a black ethnicity, a decreased preoperative renal function, a high preoperative bilirubin level, a preoperative comorbid lung disease, bilateral lung surgery, the use of intraoperative or postoperative extracorporeal membrane oxygenation (ECMO), and the ischemic time were identified as risk factors for AKI development [61]. Xue et al. examined the development of AKI of AKIN stage 1 or higher within 1 week after lung transplantation in 88 patients and found an AKI incidence of $53.4 \%$. The proposed risk factors included aging, preoperative hypertension, an intraoperative low mean blood pressure, the intraoperative use of vasopressors, the intraoperative use of aprotinin, the use of intraoperative or postoperative ECMO, and a comorbid postoperative infection [60]. In an investigation of the onset of AKI classified by the RIFLE criteria as "Risk" or more severe within 1 week after lung cancer surgery, Licker et al. reported an AKI incidence of $6.8 \%$. A low preoperative forced expiratory volume in $1 \mathrm{~s}$ (FEV $1.0 \%$, a high ASA score, and the duration of the anesthesia were identified as risk factors. The ASA score refers to the physical status assessment score advocated by the American Society of Anesthesiologists [62].

\section{Bariatric surgery}

Bariatric surgery has recently become a popular surgical intervention for severe obesity, mainly in the West. As obesity itself triggers renal impairment, multiple studies have examined the development of AKI following bariatric surgery [63-65]. In a cohort of 590 patients, Morgan and Ho reported that AKI of AKIN stage 1 or higher had developed in 103 patients, which represented an incidence of $17.5 \%$; the male gender, preoperative hypertension, and a high preoperative APACHE II score were identified as risk factors [64]. In a report of the outcomes in 1227 patients who underwent bariatric surgery at the Mayo Clinic between 2004 and 2011, the incidence of AKI (defined as a serum creatinine $[\mathrm{sCr}]$ increase of $0.3 \mathrm{mg} / \mathrm{dL}$ within $72 \mathrm{~h}$ ) was $5.8 \%$; the preoperative BMI and diabetes were identified as risk factors for AKI development [65]. 


\section{Colorectal surgery}

Causey et al. examined the development of AKI following colorectal surgery in a cohort of 339 patients who underwent colorectal surgery between 2001 and 2009 [66]. The incidence of AKI (defined as a postoperative increase in $\mathrm{sCr}$ of $\geq 50 \%$ from baseline) was $11.8 \%$; intraoperative red blood cell transfusion was identified as a risk factor.

\section{Literature review}

PubMed was searched for relevant studies published between March 2011 and December 2015, and papers related to the present CQ were identified from the search results. The literature published before March 2011 was referenced from the KDIGO Clinical Practice Guideline for AKI.

\section{CQ3-3: What should be assessed as risk factors for AKI development in heart failure?}

Recommendation: Factors such as aging, renal impairment, and cardiac dysfunction should be assessed as risk factors.

Strength of recommendation: 2

Quality of evidence: C

\section{Summary of evidence}

Among the 11 identified observational studies that included the development of AKI as an outcome, 5 were multicenter studies involving more than 1000 subjects. In multivariate analyses, the following risk factors were found to be significantly associated with AKI: comorbid CKD (4 studies), aging (4 studies), diabetes (3 studies), and cardiac dysfunction (3 studies). Other factors found to be associated with AKI were the diuretic resistance, hypotension (defined as a systolic blood pressure $<90 \mathrm{mmHg}$ ), and elevated urinary neutrophil gelatinase-associated lipocalin (NGAL) (2 studies each).

\section{Commentary \\ Background}

In cardiovascular medicine, AKI has been recognized as worsening renal function (WRF) in heart failure patients. The interaction between heart failure and kidney failure has recently been defined as cardiorenal syndromes (CRS), which are classified into five types [67, 68]. Among them, AKI associated with acute heart failure is classified as CRS type 1. Furthermore, AKI caused by acute heart failure is considered to exacerbate the heart failure, causing a vicious cycle and a poor survival prognosis for patients with CRS type 1 [69]. Therefore, it is clinically crucial to identify the incidence and risk factors of CRS type 1 .

\section{Incidence of AKI in acute heart failure}

Studies have found an inconsistent incidence of AKI in acute heart failure due to differing definitions of AKI. A retrospective cohort study in 2010 by Amin et al. featured 2098 enrolled patients (the largest cohort to date). In this study, the incidence of AKI-defined as an increase in $\mathrm{sCr}$ of $\geq 0.3 \mathrm{mg} / \mathrm{dL}$ during hospitalization-was 18.7\% [70]. However, in a retrospective cohort study ( $n$ $=1010)$ in 2013 by Wang et al. that defined AKI as stage 1 or higher according to the AKIN classification, the incidence of AKI was $32.2 \%$ [71], while in a prospective cohort study $(n=1005)$ in 2012 by Zhou et al. that defined AKI according to the RIFLE classification, the AKI incidence was $44.3 \%$ [72]. Subsequent studies by Soyler et al. [73] that defined AKI as an increase in $\mathrm{sCr}$ of $\geq$ $0.3 \mathrm{mg} / \mathrm{dL}$ within $48-72 \mathrm{~h}$ after hospital admission, and by Tung et al. [74] that examined AKIN stage $\geq 1$ AKI patients hospitalized for ST-segment elevation myocardial infarction (STEMI), reported AKI incidences of 19.0 and $19.6 \%$, respectively. In a study that examined the differences in the incidence of AKI based on the different definitions of AKI, Li et al. reported that the incidences of AKI in a cohort of patients hospitalized for acute heart failure $(n=1498)$ according to the RIFLE, AKIN, and KDIGO criteria were $32.1,34.7$, and $38.9 \%$, respectively [8]. Based on the above studies, the incidence of AKI in heart failure is considered to range between 20 and $40 \%$.

\section{Risk factors for AKI development in acute heart failure}

Observational studies have identified a number of risk factors for AKI following heart failure. A recent prospective observational study demonstrated a significant association between AKI and elevated levels ( $\geq 12 \mathrm{ng} /$ $\mathrm{mL}$ ) of the tubular dysfunction marker NGAL [75]. In 11 existing observational studies, comorbid CKD, aging, comorbid diabetes, and cardiac dysfunction were identified as independent risk factors for AKI. Diuretic resistance, hypotension (defined as a systolic blood pressure $<90 \mathrm{mmHg}$ ), and an elevated urinary NGAL were also shown to be associated with AKI in two studies each (Table 6). The degree of CKD considered to present a risk of AKI development is an eGFR $<60 \mathrm{~mL} / \mathrm{min} /$ $1.73 \mathrm{~m}^{2}[70,72,74,76]$ or a $\mathrm{sCr}$ level $\geq 104 \mu \mathrm{mol} / \mathrm{L}$ $(1.17 \mathrm{mg} / \mathrm{dL})$ [71]. In terms of age, one study stated that the odds ratio for the development of AKI increased by 1.17 (95\% confidence interval 1.08-1.28) with every 10 years increase in age [70], while other studies have reported ages of $\geq 70$ years [71] and $\geq 80$ years [76] as risk factors. One study defined the degree of cardiac dysfunction considered a risk factor for AKI development as a left ventricular ejection fraction (LVEF) $<40 \%$ [70], while others set it at LVEF $<45 \%$ or a NYHA class IV $[71,72]$. The extent of diuretic resistance that is considered a risk factor for AKI development has been defined 


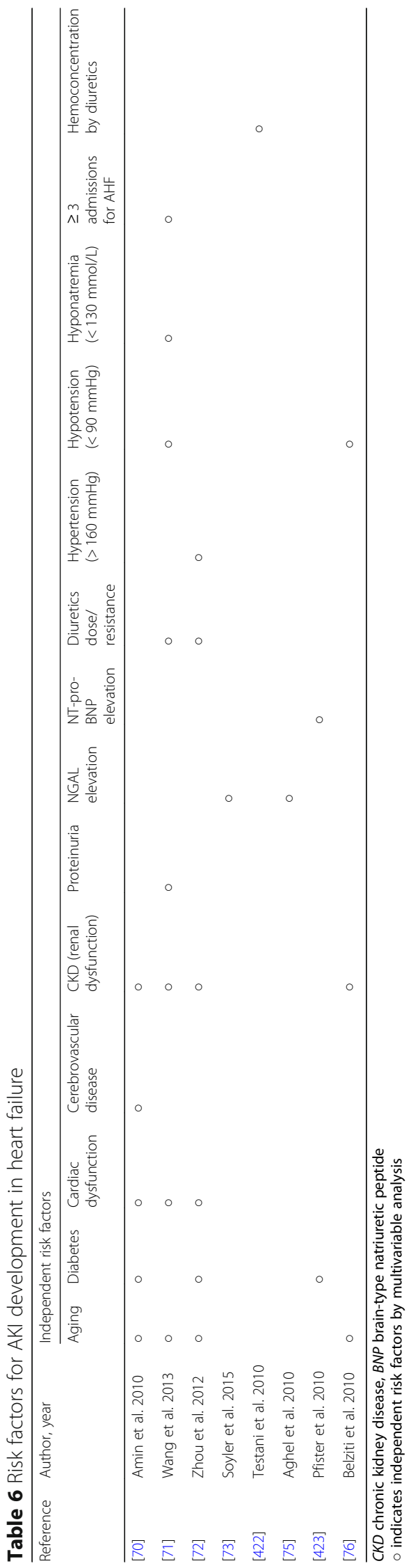


as persistent pulmonary congestion despite repeated doses of $80 \mathrm{mg}$ furosemide, the continuous administration of $240 \mathrm{mg}$ of furosemide per day, or the combination of furosemide with thiazide diuretics or an aldosterone antagonist [71, 72].

\section{Literature review}

PubMed was searched for relevant studies published between March 2011 and December 2015, and papers related to the present CQ were identified from the search results. The literature published before March 2011 was referenced from the KDIGO Clinical Practice Guideline for AKI.

\section{CQ3-4: What should be assessed as risk factors for AKI development in sepsis?}

Recommendation: Pre-existing renal dysfunction, aging, and the use of renin-angiotensin-aldosterone system inhibitors should be assessed as risk factors.

Strength of recommendation: 2

Quality of evidence: C

\section{Summary of evidence}

In six observational studies that examined the risk of AKI development in sepsis, pre-existing renal dysfunction, aging, and the use of renin-angiotensin-aldosterone system inhibitors were found to be associated with AKI development in sepsis.

\section{Commentary}

\section{Background}

Sepsis patients develop AKI frequently [34, 77]. As AKI is associated with a significantly increased mortality [78], it is crucial to assess the risk of its development in sepsis patients. In our search for observational studies aimed at identifying the risk factors for AKI development in sepsis, the numbers of relevant studies and of patients did not make for sufficiently strong evidence; however, several clinical background factors were identified as risk factors for AKI development (Table 7).

\section{Pre-existing renal dysfunction}

Pre-existing renal dysfunction is known as a risk factor for AKI development in a variety of pathologies, including sepsis. In an observational study of 992 sepsis patients by Suh et al., $57.7 \%$ of the patients developed AKI; one of the risk factors for AKI was renal dysfunction, which was defined as an eGFR $<60 \mathrm{~mL} / \mathrm{min} / 1.73 \mathrm{~m}^{2}$ (odds ratio 2.398, 95\% confidence interval [CI] 1.3014.420) [77]. In an observational study of 423 patients, Poukkanen et al. also identified pre-existing renal dysfunction as a strong risk factor for AKI in sepsis (odds ratio 7.24, 95\% CI 2.36-22.23) [79]. Moreover, Plataki et al. reported that the incidence of AKI was significantly low in individuals with a higher baseline eGFR [80]. In addition, despite the small number of patients and the lack of a significant difference, in an observational study, Medeiros et al. reported that pre-existing renal dysfunction tended to increase the risk of AKI development [81]. Therefore, whenever possible, patients must be examined for pre-existing renal dysfunction when sepsis develops. In addition, when treating septic patients with pre-existing renal dysfunction, it is necessary to monitor the renal function carefully.

\section{Aging}

Japanese society is aging rapidly. Aging is an underlying cause of age-related organ dysfunction, which creates various medical issues. Suh et al. reported that the risk of AKI development in sepsis increased with age (odds ratio 1.028, 95\% CI 1.016-1.041) [77]. Medeiros et al. reported a similar result in an observational study that found AKI to be significantly more frequent in septic patients aged over 65 (odds ratio 1.28, 95\% CI 1.12-1.89) [81]. In addition, although the risk was not assessed in a logistic regression analysis, another observational study reported that AKI patients were of a significantly higher age [82]. Therefore, the potential development of AKI must be considered while treating elderly patients with sepsis.

Table 7 Risk factors for AKI development in sepsis

\begin{tabular}{|c|c|c|c|c|c|c|c|c|}
\hline Reference & Author, year & $\begin{array}{l}\text { Pre-existing renal } \\
\text { dysfunction }\end{array}$ & Aging & RAAS inhibitors & Diabetes & $\begin{array}{l}\text { Intra-abdominal } \\
\text { infection }\end{array}$ & $\begin{array}{l}\text { Blood } \\
\text { product }\end{array}$ & Shock \\
\hline$[80]$ & Plataki et al. 2011 & $\circ$ & - & $\circ$ & $\times$ & $\circ$ & $\circ$ & - \\
\hline [77] & Suh et al. 2013 & $\circ$ & $\circ$ & $\circ$ & $\times$ & - & - & $\circ$ \\
\hline [79] & Poukkanen et al. 2013 & $\circ$ & - & - & - & - & - & - \\
\hline [81] & Medeiros et al. 2015 & $\Delta$ & $\circ$ & - & $\circ$ & - & - & - \\
\hline [83] & Chang et al. 2012 & - & - & - & $\circ$ & - & - & - \\
\hline [84] & Venot et al. 2015 & - & - & - & $x$ & - & - & - \\
\hline
\end{tabular}

RAAS renin-angiotensin-aldosterone system

$\circ$ risk, $\triangle$ risk without significance, $\times$ not risk, - not evaluated 


\section{Renin-angiotensin-aldosterone system inhibitors}

An increase in patients with hypertension has led to a corresponding increase in the number of patients using renin-angiotensin-aldosterone system inhibitors. As these drugs reduce the systemic blood pressure and dilate the efferent arterioles, they may enhance the reduction of the GFR during shock. Therefore, there is a concern that renin-angiotensin-aldosterone system inhibitors may exacerbate the risk of AKI development. In two observational studies that examined whether the use of renin-angiotensin-aldosterone system inhibitors was a risk factor for AKI development in sepsis, the risk was found to be approximately twice as high when using these drugs than when not using them [77, 80]. Therefore, when sepsis develops, the careful monitoring of potential AKI development is recommended in patients using renin-angiotensin-aldosterone system inhibitors. However, there have been no intervention trials to determine whether the withdrawal of these drugs during sepsis can prevent the development of AKI. This question needs to be examined in a RCT.

\section{Other risk factors}

In addition to the above risk factors, observational studies have also assessed obesity, comorbid diabetes, intra-abdominal bacterial infection, the use of blood products, and hypotension as potential risk factors for AKI development [77, 80, 81, 83]. However, no definitive conclusions have been reached. For instance, one study found that diabetes is not associated with the development of AKI in sepsis [84]. Further evidence needs to be collected in order to determine whether these factors increase the risk of AKI development in sepsis.

\section{Literature review}

PubMed was searched for relevant studies published between March 2011 and December 2015, and papers related to the present CQ were identified from the search results. The literature published before March 2011 was referenced from the KDIGO Clinical Practice Guideline for AKI.

\section{CQ4-1: Should hospital-acquired AKI and community-acquired AKI be differentiated?}

Recommendation: Hospital-acquired AKI has a worse survival prognosis than community-acquired AKI. In addition, the relationship between the severity and the mortality may differ between the two types of AKI. Therefore, we suggest that they should be differentiated from one another.

Strength of recommendation: Not graded

Quality of evidence: D

\section{Summary of evidence}

In a meta-analysis of eight observational studies, the mortality was significantly higher in hospital-acquired AKI than in community-acquired AKI (odds ratio 2.79, 95\% confidence interval 2.18-3.56). In studies that used the RIFLE or KDIGO criteria, community-acquired AKI featured a high rate of stage $3 \mathrm{AKI}$, while hospital-acquired AKI featured a high rate of stage $1 \mathrm{AKI}$.

\section{Commentary}

Acute kidney injury (AKI) is primarily treated with conservative therapies, such as the optimization of fluid volume or blood pressure and the avoidance of nephrotoxins; in addition, identification of the cause of the kidney injury is recommended. Therefore, it is crucial to recognize the risk factors for AKI and take steps to prevent it in order to improve its outcomes [3].

Acute kidney injury encompasses a broad spectrum of diseases and can occur in the hospital or in the community. However, although community-acquired AKI occurs frequently in low- and middle-income countries which account for roughly $85 \%$ of the world population [3], $80-90 \%$ of studies have examined hospital-acquired AKI in high-income countries [34]; few studies have compared hospital-acquired and community-acquired AKI. Hospital-acquired AKI is frequently caused by ischemia, nephrotoxins, and sepsis [85], while community-acquired AKI has been found to frequently derived from preventable causes such as dehydration, infection, and childbirth [86]. To determine the state of community-acquired AKI in low- and middle-income countries, the multinational 0by 25 initiative conducted the global snapshot study in 2015 [87].

In order to develop the present guideline, PubMed was used to identify papers that compared hospital-acquired and community-acquired AKI. Eight observational studies were identified [88-95]; among them, two defined AKI based on the RIFLE criteria [92, 93], two used the KDIGO criteria [94, 95], and the other four were published before the RIFLE and KDIGO criteria were proposed [88-91]. Four studies were conducted in high-income countries [89, 92-94], while the other four were conducted in low- and middle-income countries [88, 90, 91, 95]. In all of these studies, community-acquired AKI was associated with a lower mortality (Fig. 1) and shorter hospitalization duration. Moreover, the percentages of patients at each AKI stage (i.e., degree of severity) in the studies that used the AKIN criteria indicated that in all four studies, community-acquired AKI was more severe (i.e., with low percentages of stages 1 and 2 AKI and high percentages of stage 3 AKI), while hospital-acquired AKI showed higher percentages of mild cases (Fig. 2). 


\begin{tabular}{lrrrrrr}
\multicolumn{1}{c}{ 1st author, Year } & $\begin{array}{c}\text { Odds } \\
\text { ratio }\end{array}$ & $\begin{array}{c}\text { Lower } \\
\text { limit }\end{array}$ & $\begin{array}{c}\text { Upper } \\
\text { limit }\end{array}$ & Z-Value & p-Value \\
Loo CS et al, 1995 & 2525 & 1.223 & 5.213 & 2.503 & 0.012 \\
Obialo Cl, et al, 2000 & 2718 & 1.017 & 7.267 & 1.993 & 0.046 \\
Sesso R et al, 2004 & 2792 & 1.774 & 4.394 & 4.439 & 0.000 \\
Wang Y et al, 2007. & 5.631 & 3.050 & 10.396 & 5.525 & 0.000 \\
Schissler MMet al, 2013 & 4.327 & 2487 & 7.527 & 5.185 & 0.000 \\
Der Mesropian PJ et al, 2014 & 1.481 & 0.983 & 2232 & 1.878 & 0.060 \\
Wornacott A et al, 2014 & 3.056 & 2293 & 4.072 & 7.627 & 0.000 \\
XuXet al, 2015 & 2409 & 2072 & 2801 & 11.434 & 0.000 \\
$\quad$ Overall & 2788 & 2183 & 3.560 & 8.215 & 0.000 \\
$\quad$ & & & & &
\end{tabular}

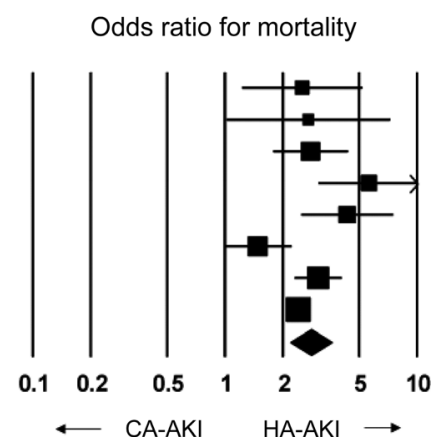

Fig. 1 In-hospital mortality in CA-AKI vs HA-AKI. CA-AKI: community-acquired acute kidney injury, HA-AKI: hospital-acquired acute kidney injury

Thus, the above-cited studies demonstrate that hospital-acquired AKI and community-acquired AKI have different clinical pictures, as shown in Table 8 . The relationship between the severity and mortality may differ between hospital-acquired AKI and community-acquired AKI; therefore, we suggest that they are discriminated from one another.

However, all the studies used were conducted outside Japan. A further investigation comparing hospital-acquired AKI and community-acquired AKI in Japan is necessary.

\section{Literature review}

PubMed was searched for relevant studies published up to December 2015, and papers that compared hospital-acquired and community-acquired AKI were identified from the search results.

\section{CQ4-2: Should septic AKI and non-septic AKI be discriminated from each other?}

Recommendation: Septic AKI may lead to a higher mortality than non-septic AKI; therefore, we suggest that they should be discriminated from each other.

Strength of recommendation: Not graded

\section{Quality of evidence: D}

\section{Summary of evidence}

In a meta-analysis based on nine observational studies, compared to non-septic AKI, septic AKI resulted in a higher in-hospital mortality (odds ratio 2.48 , 95\% confidence interval 1.76-3.49) and a higher ICU mortality (odds ratio 1.60, 95\% confidence interval 1.52-1.69). Although studies that assessed the in-hospital mortality featured publication bias, no such bias was observed related to ICU mortality.

\section{Commentary}

In a report of a large-scale prospective observational study conducted at 54 centers in 23 countries [34], the cause of acute kidney injury (AKI) in the intensive care unit (ICU) was a septic shock in $47.5 \%$ of cases and cardiogenic shock in $26.9 \%$ of cases. In a large-scale multinational multicenter prospective observational study published in 2015 [96], AKI occurred in 57.3\% of ICU patients; the cause of AKI was sepsis in $40.7 \%$ of patients and cardiogenic shock in $13.2 \%$ of patients. In Japanese epidemiology, the Diagnosis Procedure Combination (DPC) database has been used to examine AKI patients

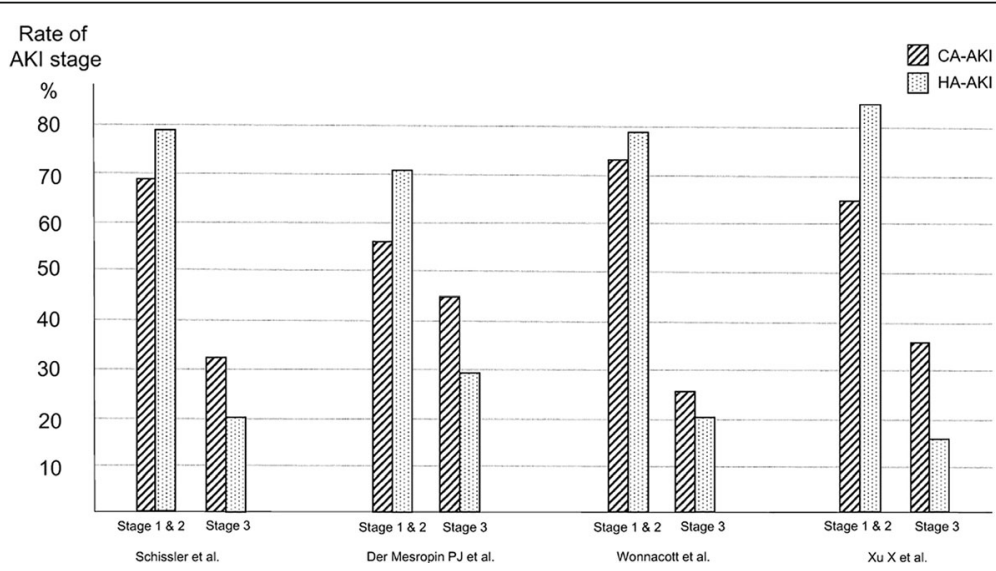

Fig. 2 Rate of AKI stage in CA-AKI vs HA-AKI. CA-AKI: community-acquired acute kidney injury, HA-AKI: hospital-acquired acute kidney injury 
Table 8 Differences between CA-AKI and HA-AKI

\begin{tabular}{lll}
\hline & Hospital-acquired AKI & Community-acquired AKI \\
\hline Mortality & High & Low \\
Severity & Stage 1 and 2> stage 3 & Stage 3 > stage 1 and 2 \\
\hline
\end{tabular}

who underwent continuous renal replacement therapy (CRRT) [97]. Among these patients, the most common causes of AKI were cardiovascular disease and other medical diseases, which accounted for approximately half of the patients, followed by sepsis and cardiovascular surgery; compared to all other causes, mortality was low only for cardiovascular surgery.

In developing the present guideline, PubMed was used to identify papers which compared septic and non-septic AKI. Nine observational studies were identified [78, 98105]; seven of these studies were prospective, while two were retrospective. One of these studies was a retrospective study by Bagshaw et al. which utilized the Australian and New Zealand Intensive Care Society (ANZICS) database [78]; the study featured 14,039 septic AKI patients and 29,356 non-septic AKI patients, a prominently large number of patients compared to other studies. Seven studies compared in-hospital mortality [78, 98-103], while five studies compared ICU mortality $[78,100,101,104,105]$.

As for AKI diagnostic criteria, six studies used the RIFLE criteria [78, 99-103]; the remaining three studies [98, 104, 105] were published before the RIFLE criteria were proposed. Percentages of patients by RIFLE criteria severity were listed in four studies $[78,99,100,102]$; Risk was the most common level of severity in one study [100], while Injury was the most common in two studies [78, 99], and Failure was the most common in one study [102]. Causes of sepsis were demonstrated in two studies [99, 101]; in these studies, sepsis was caused by intrathoracic infections (such as pneumonia) and intra-abdominal infections in approximately 30 and $25 \%$ of cases, respectively, thus accounting for more than half of all cases. The severities of patients' illnesses were assessed with the APACHE II, SAPS, and SAPS II severity scores in eight studies [78, 98-101, 103-105]; in all of these studies, septic AKI was more severe than non-septic AKI.

Figure 3 shows the results of a meta-analysis of seven studies which compared in-hospital mortality. In-hospital mortality and ICU mortality may both be higher for septic AKI than for non-septic AKI; therefore, we suggest that the two forms of AKI be discriminated from each other. Septic AKI should be handled in specific ways, such as the admission of patients to the ICU depending on severity, consideration of hemodynamic monitoring, and maintaining fluid volume and renal perfusion pressure.

\section{Literature searches}

Searches were conducted on PubMed for literature published up to November 2015. Papers which compared septic and non-septic AKI were identified from the search results.

\section{CQ4-3: Should renal AKI and pre-renal AKI be differentiated?}

Recommendation: The in-hospital mortality may be higher in renal AKI than in pre-renal AKI; therefore, we suggest that they should be differentiated from one another.

Strength of recommendation: Not graded

Quality of evidence: D

\section{Summary of evidence}

In a meta-analysis of ten observational studies, the in-hospital mortality from renal AKI was higher than that from pre-renal AKI (odds ratio 3.63, 95\% confidence interval 1.68-7.83). A significant publication bias was present.

\section{Commentary}

Acute kidney injury (AKI) is classified as either pre-renal, renal (intrinsic), or post-renal. Pre-renal AKI is considered as azotemia resulting from a decreased renal perfusion pressure; conceptually, it is a form of renal impairment with no renal tissue damage, in which the renal function can recover rapidly with early treatment. There are two conceivable approaches to the differentiation of pre-renal

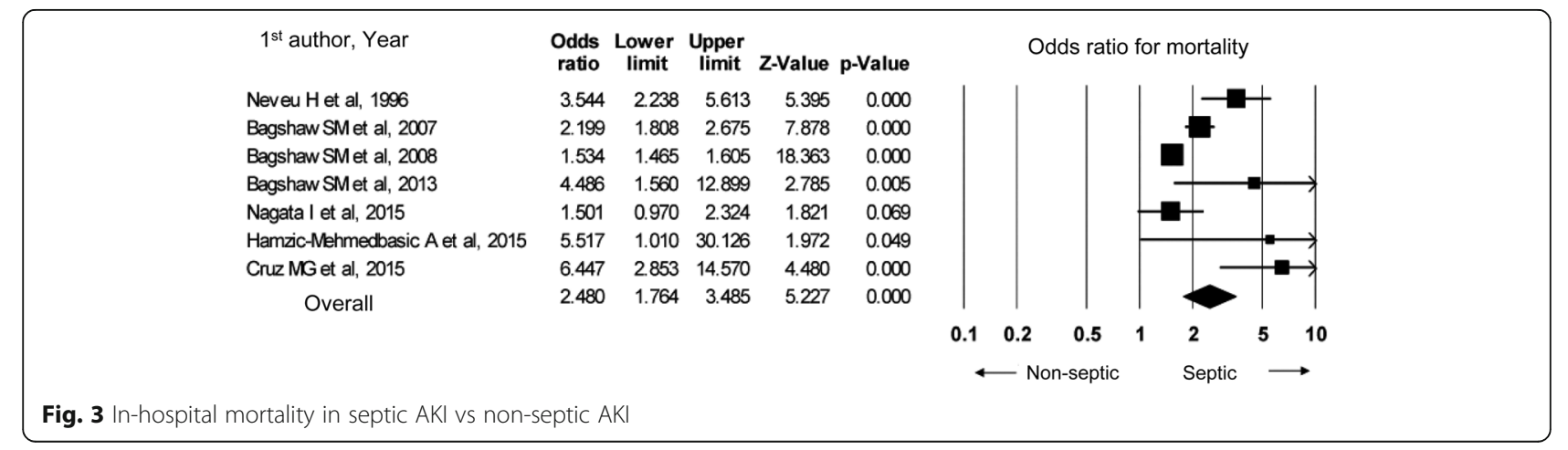


AKI from renal AKI. The first approach is to comprehensively assess whether the AKI is pre-renal or renal based on an assessment of the cause of the AKI, hemodynamics, and urinalyses with measuring factors such as the body weight change, vital signs, urine osmolality, fractional excretion of sodium (FENa), fractional excretion of urea nitrogen (FEUN), and urinary sediment. The second approach is to determine whether the renal function recovers immediately after fluid resuscitation. If the renal function recovers within 2-3 days after appropriate fluid resuscitation, the AKI is considered to be volume-responsive, which allows for clinical classification as pre-renal AKI. If the renal function does not recover despite fluid resuscitation, the AKI is considered to be volume-unresponsive, which corresponds to renal AKI. However, when a continued or prolonged reduced renal perfusion pressure results in renal parenchymal injury, or when the reduced renal perfusion pressure is accompanied by a low cardiac output, sepsis, or liver failure, the renal function does not necessarily recover with fluid resuscitation alone [106]. Therefore, even if the AKI is initially assessed as pre-renal, a second test should be performed within 3 days. However, even if the AKI is assessed as pre-renal, a mild elevation in the urinary biomarkers can sometimes be suggestive of a renal tissue injury [107]. As AKI is known to be involved in injuries to multiple organs, including the heart and lungs, even pre-renal AKI may affect the survival prognosis.

Many studies have reported that the in-hospital mortality is lower in volume-responsive AKI-in which the renal function recovers within 3 days of interventionthan in volume-unresponsive AKI. In a recent AKI cohort study of 283 patients in intensive care units (ICUs) at multiple hospitals, the in-hospital mortality rates for non-AKI, volume-responsive AKI, and renal AKI were 23.8, 29.6, and 38.9\%, respectively; thus, renal AKI showed the worst outcomes [108]. However, in a study that evaluated AKI based on its underlying causes at diagnosis, the in-hospital mortality was $27.3 \%$ in pre-renal AKI versus $19.3 \%$ in intrinsic AKI; although the difference was not significant, pre-renal AKI tended to have worse outcomes [109].

To develop the present guideline, PubMed was used to identify papers that compared renal and pre-renal AKI in order to assess the difference in the survival outcomes. Ten cohort studies were identified; among them, three differentiated between pre-renal and renal AKI based on the underlying causes of AKI and the urine findings at diagnosis [109-111], while seven differentiated between pre-renal and renal AKI based on the volume responsiveness [107, 108, 112-116]. In our meta-analysis, the in-hospital mortality was significantly higher in renal AKI than in pre-renal AKI (Fig. 4).
Based on the above, pre-renal AKI and renal (intrinsic) AKI have different survival outcomes; therefore, we suggest that they should be distinguished from one another.

\section{Literature review}

PubMed was searched for relevant studies published up to August 2015, and papers related to the present CQ were identified from the search results.

\section{CQ5-1: Should urinary biomarkers be used for the early diagnosis of AKI?}

Recommendation: Due to their potential utility in the early diagnosis of AKI, we suggest measuring the urinary NGAL and L-type fatty acid-binding protein (L-FABP). However, the utility of the urinary cystatin $\mathrm{C}$ is limited; therefore, we cannot make a recommendation about its use.

Urinary NGAL and urinary L-FABP:

Strength of recommendation: 2

Quality of evidence: B

Urinary cystatin $C$ :

Strength of recommendation: Not graded

Quality of evidence: C

\section{Summary of evidence}

Multiple systematic reviews/meta-analyses have found the urinary NGAL and L-FABP to serve as useful markers for the early diagnosis of AKI. However, future clinical trials that compare AKI interventions based on the conventional diagnostic method using the serum creatinine levels with those based on diagnoses made with urinary biomarkers are necessary to examine whether novel urinary biomarkers are truly useful for the diagnosis of AKI.

Only one systematic review/meta-analysis has assessed the utility of the urinary cystatin $\mathrm{C}$; therefore, firm conclusions as to its utility for the early diagnosis of AKI cannot be made.

\section{Commentary}

The pathological condition previously recognized as acute renal failure (ARF) is now broadly understood to pose a risk of death at an earlier or milder stage than failure. This has prompted a paradigm shift from ARF to acute kidney injury (AKI). However, with the present method of diagnosis, which is based on the identification of an increased level of serum creatinine $(\mathrm{sCr})$ and a reduced urine output, interventions are often mistimed; therefore, there is an urgent need for the clinical application of more sensitive biomarkers. The early diagnosis of AKI enables earlier consultation with a nephrologist, appropriate management of the renal hemodynamics, and the avoidance of exposure to nephrotoxins. Therefore, we examined whether urinary biomarkers should be 


\begin{tabular}{|c|c|c|c|c|c|}
\hline ar & $\begin{array}{l}\text { Odds } \\
\text { ratio }\end{array}$ & $\begin{array}{c}\text { Lower } \\
\text { limit }\end{array}$ & $\begin{array}{c}\text { Upper } \\
\text { limit }\end{array}$ & e & \\
\hline aufman $\mathrm{J}$ et al, 1 & 15.600 & 3.497 & 69.587 & 3.601 & \\
\hline Santacruz F et al, 1996 & 208 & .946 & 0.886 & 870 & \\
\hline ickolas TL et al, 2008 & 2.554 & 0.638 & 10.219 & 325 & \\
\hline Soto $\mathrm{Ke}$ & 4.017 & 1.419 & 1.372 & 619 & \\
\hline Singer $\mathrm{E}$ & 3.712 & 64 & 08 & 502 & \\
\hline oi Ke & 85 & 53 & & 251 & \\
\hline ark M & 34 & 54 & 1057.201 & 902 & \\
\hline Yang $\mathrm{F}$ et al, 2014 & 627 & 35 & 175 & 1.457 & \\
\hline Koyner JL & 944 & 60 & 3.881 & 064 & \\
\hline erinel $S$ et al, 2 & & & 21 & 573 & \\
\hline Overall & 3.627 & 1.679 & 7.834 & 3.279 & \\
\hline
\end{tabular}

Fig. 4 In-hospital mortality in renal AKI vs pre-renal AKI

used for the early diagnosis of AKI based on a relatively large number of studies on AKI in adult patients having received cardiovascular surgery and those in intensive care units (ICUs).

Neutrophil gelatinase-associated lipocalin (NGAL) is a low molecular weight protein (molecular weight, approx. $25,000)$ that belongs to the lipocalin protein family and is secreted by activated neutrophils. In addition to inducing kidney development and possessing renoprotective and antibacterial effects, NGAL is also expressed in the distal nephron in kidney injury. Multiple systematic reviews/meta-analyses have found the urinary NGAL to be useful for the early diagnosis of AKI [117-122]. Among the studies cited in these systematic reviews/meta-analyses, 16 (for a total of 2194 patients) were related to the present CQ (Table 9) [123-138]. The subjects consisted of patients who had undergone cardiovascular surgery (14 studies, 1531 patients in all) [123, 124, 126-130, 132-138] and ICU patients (two studies, 663 patients in all) $[125,131]$. The majority of these studies defined AKI according to the RIFLE or AKIN criteria (i.e., an increase in $\mathrm{SCr}$ ) or to criteria conforming to the RIFLE or AKIN ones. A total of 549 patients (25\%) were diagnosed with AKI. In an assessment of the early diagnostic capacity of the urinary NGAL over the 6-h period immediately after surgery or ICU admission, the area under the receiver operating characteristic curve (AUC) was 0.50-0.98 (0.77 with an unweighted mean). In $75 \%(12 / 16)$ of the studies, the AUC was $\geq 0.70$, thus showing moderate or better diagnostic accuracy; therefore, the urinary NGAL was found to be useful for the early diagnosis of AKI. However, the clinical studies related to the present CQ raised several issues about the clinical application of the urinary NGAL, including the following: some studies did not use officially approved measurement methods; multiple measurement methods were used, and they were not standardized; there was no set cutoff value; urinary tract infections and urologic diseases increase the urinary NGAL levels [139]; and there are very few relevant clinical studies of Japanese subjects.

The L-type fatty acid-binding protein (L-FABP) is a low molecular weight protein (molecular weight, approx. $14,000)$ localized in the cytoplasm of human renal proximal tubular cells. By binding to free fatty acids and transporting them to mitochondria and peroxisomes, the L-FABP promotes beta-oxidation, contributes to energy production, and helps to maintain homeostasis. When the proximal tubule is subjected to ischemia or oxidative stress, the expression of the L-FABP is enhanced and its urinary excretion increases. The urinary L-FABP has been demonstrated to be useful for the early diagnosis of AKI $[117,118,140]$ by multiple systematic reviews/ meta-analyses. Among the studies cited in these systematic reviews/meta-analyses, seven (for a total of 2416 patients) were related to the present CQ (Table 10) [136, 141-146]. The subjects consisted of patients who had undergone cardiovascular surgery (three studies, $271 \mathrm{pa}-$ tients in all) $[136,141,146]$ and ICU patients (four studies, 2145 patients in all) [142-145]. These studies generally defined AKI according to the RIFLE or AKIN criteria (i.e., an increase in $\mathrm{sCr}$ ). A total of 298 patients (12\%) were diagnosed with AKI. In an assessment of the early diagnostic capacity of the urinary L-FABP over the 12-h period immediately after surgery or ICU admission, the AUC was 0.70-0.95 (0.81 with an unweighted mean). In all seven studies, the AUC was $\geq 0.70$, thus showing moderate or better diagnostic accuracy; therefore, the urinary L-FABP was found to be useful for the early diagnosis of AKI. However, the timing of the urinary L-FABP measurement must be chosen carefully according to the different AKI etiologies. Measuring reagents for the L-FABP are available in Japan; these are standardized and covered by public health insurance.

Cystatin $\mathrm{C}$ is a low molecular weight protein (molecular weight, approx. 13,000) produced by nucleated cells all over the body that inhibits the cell injury caused by 


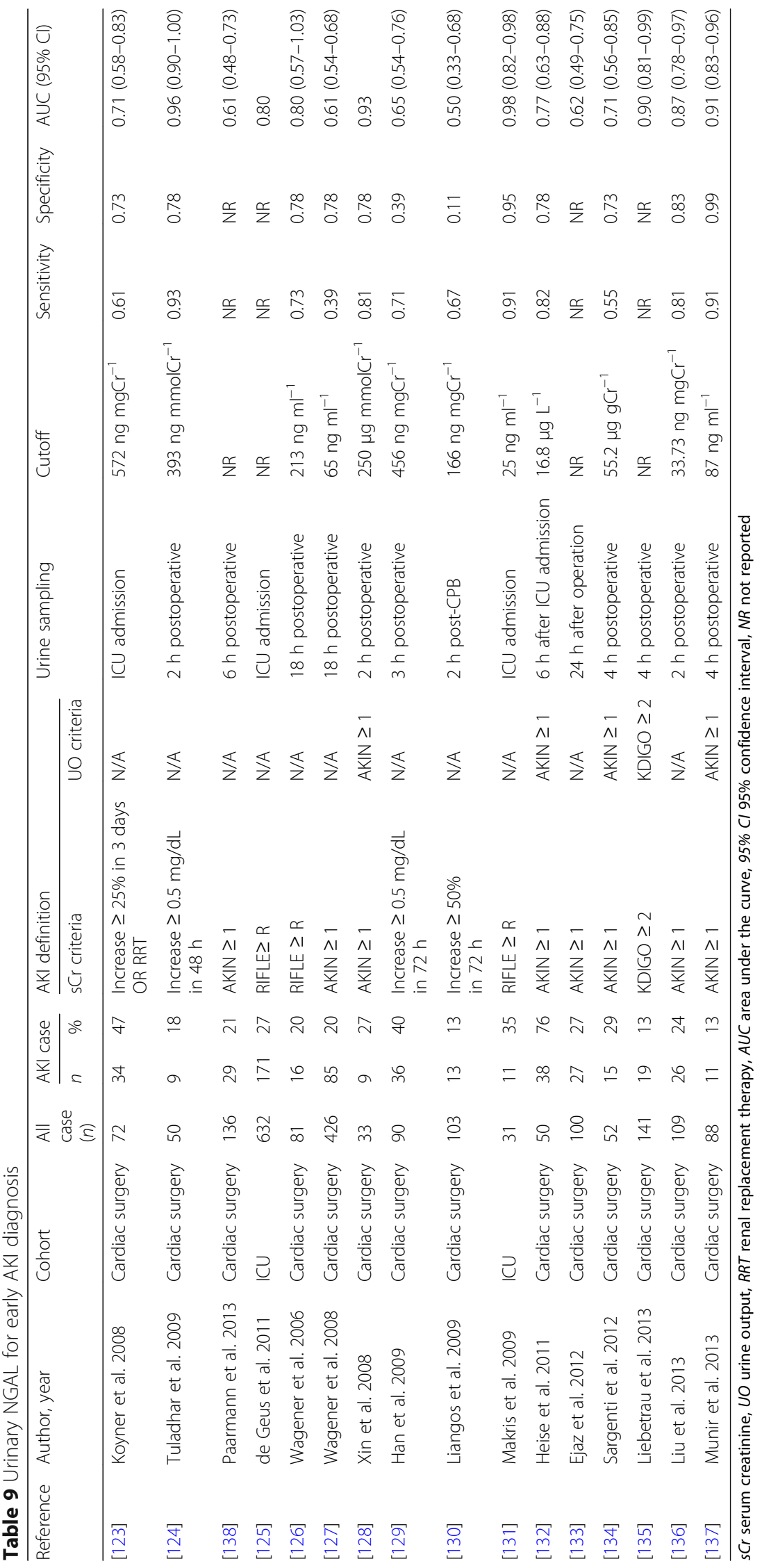




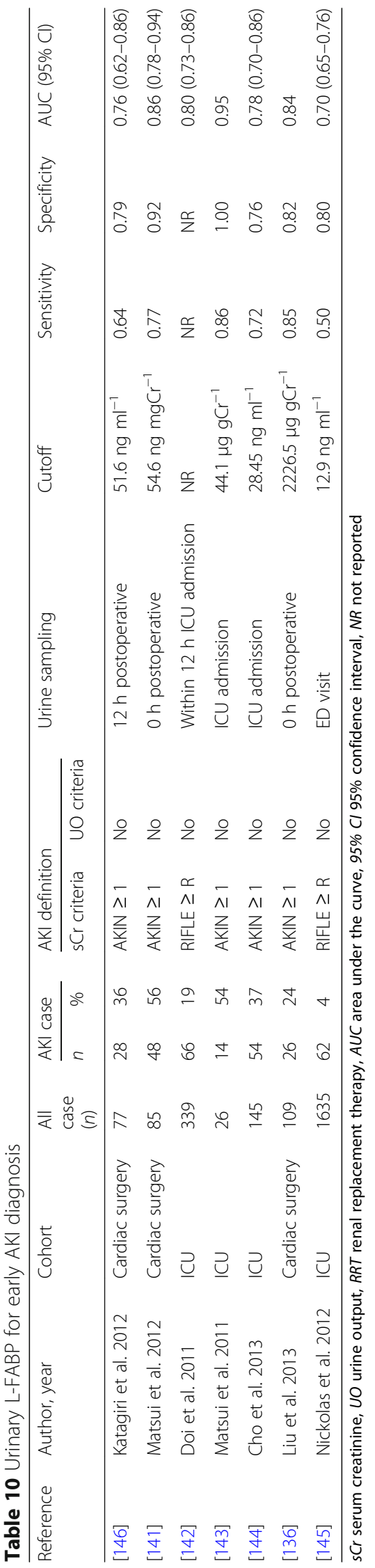


cysteine proteases. After being secreted outside the cells, cystatin C is filtered by the glomerulus; $99 \%$ of it is then absorbed by the proximal tubule and catabolized. Therefore, tubular injuries are affected by the reabsorption of cystatin $\mathrm{C}$; consequently, the cystatin $\mathrm{C}$ concentration in urine has been examined as a biomarker of AKI. There has been one systematic review/meta-analysis of the early diagnostic capacity of the urinary cystatin C [147]; the latter cited six studies [116, 123, 130, 132, 148, 149]. In a pool analysis of four studies that used urinary creatinine-adjusted data [116, 130, 148, 149], the sensitivity for the early diagnostic capacity of urinary cystatin $\mathrm{C}$ was 0.52 , the specificity was 0.70 , and the AUC was 0.64 (95\% confidence interval $0.62-0.66$ ); therefore, the diagnostic accuracy was low, indicating that the urinary cystatin $\mathrm{C}$ is of limited utility for the early diagnosis of AKI. The measurement of cystatin $C$ in urine samples is not covered by public health insurance in Japan. Although the urinary albumin has been reported to be a potential biomarker of early AKI [150], the number of relevant studies is limited; thus, the utility of the urinary albumin as a biomarker remains unknown.

As described above, the urinary NGAL and L-FABP have been identified as useful biomarkers for the early diagnosis of AKI. However, future clinical trials that compare AKI interventions based on the conventional diagnostic method using the serum creatinine levels with those based on diagnoses made with urinary biomarkers are necessary to examine whether novel urinary biomarkers are truly useful for the diagnosis of AKI.

\section{Literature review}

PubMed was searched for relevant studies published up to August 2015, and papers related to the present CQ were identified from the search results.

Search query: ((“"acute kidney injury"[MeSH Terms] OR "acute kidney injury" $[\mathrm{tw}] \mathrm{OR}$ "acute renal failure" $[\mathrm{tw}]$ ) AND ("biological markers"[MeSH Terms] OR "biological markers"[All Fields] OR "biomarker"[All Fields])) AND ("diagnosis"[Subheading] OR "diagnosis"[All Fields] OR "diagnosis"[MeSH Terms])) AND (Meta-Analysis[PT] OR systematic[SB]).

\section{CQ5-2: Should urinary biomarkers be used to predict the AKI severity and mortality?}

Recommendation: Although the urinary NGAL is of limited utility in predicting the AKI severity and mortality, we suggest measuring urinary NGAL. The utilities of the urinary L-FABP and cystatin $C$ in this regard are unclear.

Urinary NGAL:

Strength of recommendation: 2

Quality of evidence: C

Urinary L-FABP and urinary cystatin $C$ :

Strength of recommendation: Not graded

\section{Quality of evidence: D}

\section{Summary of evidence}

Multiple systematic reviews/meta-analyses about the use of the urinary NGAL to predict the AKI severity and survival outcomes have suggested that the urinary NGAL is potentially useful, albeit limitedly, in predicting the severity in relation to death and renal replacement therapy initiation. The number of studies on the urinary L-FABP and cystatin C is limited; therefore, their utilities in predicting the AKI severity in relation to death and renal replacement therapy initiation are unclear.

\section{Commentary}

Acute kidney injury (AKI) has been shown to be involved not only in short-term kidney injury but also in the subsequent renal outcomes and mortality. Therefore, the prediction of these outcomes is of clinical importance. In addition to the serum creatinine $(\mathrm{sCr})$, the cystatin $\mathrm{C}$, and the estimated glomerular filtration rate (eGFR), all of which reflect the renal function, studies have examined the urinary NGAL, NAG, L-FABP, and cystatin $C$ as biomarkers of kidney injury [117-119, 121, $147,151]$. The results of several systematic reviews and meta-analyses of these studies have been reported in recent years.

Regarding the utility of the urinary NGAL for the prediction of the renal outcomes and mortality, multiple systematic reviews/meta-analyses have indicated that the urinary NGAL could help to predict the AKI severity in relation to death and the initiation of renal replacement therapy (RRT). In a meta-analysis of nine studies (a total of 1948 patients), the odds ratios for RRT requirement and in-hospital mortality based on increased urinary and serum NGAL levels were 12.9 and 8.8, respectively [122]. Meanwhile, in an analysis of 13 studies (a total of 1079 patients) on the recovery of the renal function after kidney transplantation, the NGAL was shown to be a useful predictor of AKI, with an area under the curve (AUC) of receiver operating characteristic (ROC) of 0.87 [120]. However, these meta-analyses examined both urine and blood specimens; therefore, the results should be interpreted cautiously. Another cautionary point is that reports of NGAL measurements have used multiple measurement kits with different upper and lower limits.

In a meta-analysis of studies that used the urinary L-FABP to predict the need for RRT (three studies, 436 patients in all) and the in-hospital mortality (three studies, 561 patients in all), while no significant difference was observed in the need for RRT, the in-hospital mortality odds ratio was $13.7(p=0.008)$ [140]. Although measurement of the urinary L-FABP is covered by public health insurance in Japan, in principle, it can only be calculated once every 3 months. In addition, the urinary 
L-FABP has been reported to be elevated in patients with diabetes and chronic kidney diseases.

Regarding the examinations of the cystatin $\mathrm{C}$, a meta-analysis of seven studies (a total of 2941 patients) showed that high levels of cystatin $\mathrm{C}$ are a risk factor for death (odds ratio 2.3) [152]. However, both blood and urine samples were examined in this analysis. Although measurement of the serum cystatin $C$ is covered by public health insurance in Japan, that of the urinary cystatin $\mathrm{C}$ is not.

Other AKI markers that have been used in the past include the NAG, which increases by the release from the tubular epithelium brush border into the urine, and the $\beta 2$ microglobulin ( $\beta 2 M G)$ and $\alpha 1$ microglobulin ( $\alpha 1 M G)$, which will be increased by impaired tubular epithelial cell reabsorption. However, these markers are fraught with problems, as the samples are unstable (e.g., they are subject to changes in the urinary $\mathrm{pH}$ ) and they are easily affected by the serum concentration of $\beta 2 M G$ and a1MG. In addition, the levels of these markers can be increased by tubular disorders caused by proteinuria associated with glomerular injuries.

In measuring and assessing these urinary biomarkers, one should be cautious about the timing of the sample collection. In surgeries that use cardiopulmonary bypasses, the levels of the urinary NGAL and L-FABP increase 2-6 h after the surgery before declining gradually. Other biomarkers' levels also peak within a relatively short period and then decline. Therefore, if the timing of the AKI development is unknown, it is necessary to consider whether the testing was performed at the appropriate time for measurement.

\section{Literature review}

PubMed was searched for relevant studies published up to August 2015, and papers related to the present CQ were identified from the search results.

Search query: (((“acute kidney injury”[MeSH Terms] OR "acute kidney injury" [tw] OR "acute renal failure" [tw]) AND ("biological markers"[MeSH Terms] OR "biological markers"[All Fields] OR "biomarker"[All Fields])) AND ("diagnosis"[Subheading] OR "diagnosis"[All Fields] OR "diagnosis"[MeSH Terms])) AND (Meta-Analysis[PT] OR systematic[SB]).

\section{CQ5-3: Should urinary biomarkers be used to differentiate pre-renal AKI from renal AKI?}

Recommendation: Although the urinary NGAL is of limited utility for the differentiation of pre-renal AKI from renal AKI, we suggest measuring urinary NGAL. The utilities of the urinary NAG, L-FABP, and cystatin C in this regard are unknown.

Urinary NGAL:

Strength of recommendation: 2
Quality of evidence: $\mathrm{C}$

Urinary NAG, L-FABP, and cystatin C:

Strength of recommendation: Not graded

Quality of evidence: D

\section{Summary of evidence}

Observational studies have reported that the urinary NGAL is mildly elevated in pre-renal AKI and highly elevated in renal AKI; therefore, the urinary NGAL can be useful in the differentiation of pre-renal from renal AKI. However, the measurement points and cutoff values have not yet been determined. Therefore, we recommend incorporating other laboratory findings and physical findings to differentiate pre-renal from renal AKI. The utility of other urinary biomarkers in this regard is unknown.

\section{Commentary}

The conventional indicators for the differentiation of pre-renal acute kidney injury (AKI) and renal AKI include the urine osmolality, the fractional excretion of sodium (FENa), the fractional excretion of urea nitrogen (FEUN), and the urine sediment findings; however, none of these tests can be considered sufficiently sensitive or specific. There have been no systematic reviews or meta-analyses of studies on the use of urinary biomarkers for the differentiation of pre-renal from renal AKI; only a small number of observational studies are available. In multiple studies in which patients diagnosed with AKI were divided into patients with pre-renal or renal AKI, the degree of elevation of the urinary NGAL was found to be potentially useful for the differentiation of these two types of AKI.

In Nickolas et al.'s examination of the urinary NGAL in 635 patients hospitalized after emergency room visits, the mean urinary NGAL level was significantly higher in renal AKI patients $(n=30 ; 416 \pm 387 \mu \mathrm{g} / \mathrm{gCr})$ than in pre-renal AKI patients $(n=88 ; 30.1 \pm 92.0 \mu \mathrm{g} / \mathrm{gCr})$ [112]. In a report of 145 hospitalized patients by Singer et al., the median urinary NGAL level was significantly higher in renal AKI patients $(n=75 ; 255.6 \mu \mathrm{g} / \mathrm{L}[98.5-872.9 \mu \mathrm{g} /$ L]) than in pre-renal AKI patients $(n=32 ; 31.3 \mu \mathrm{g} / \mathrm{L}$ [15.9-75.5 $\mu \mathrm{g} / \mathrm{L}]$ ) [115]. Moreover, a urinary NGAL cutoff level of $104 \mu \mathrm{g} / \mathrm{L}$ yielded a high sensitivity (0.75), high specificity (0.88), and high positive likelihood ratio (5.97) for the diagnosis of renal AKI. In a report by Seibert et al., the urinary NGAL levels within 3 days of hospital admission were significantly higher in renal AKI patients $(458.1 \pm 695.3 \mathrm{ng} / \mathrm{mL})$ than in pre-renal AKI patients $(64.8 \pm 62.1 \mathrm{ng} / \mathrm{mL})$. In addition, a urinary NGAL cutoff level of $52 \mathrm{ng} / \mathrm{mL}$ yielded high sensitivity (0.75), high specificity (0.72), and a high area under the receiver operating characteristic curve (AUC 0.89) for the diagnosis of renal AKI [153]. 
Although one study found that the urinary NGAL levels of pre-renal AKI patients were not elevated [154], other studies have reported that the urinary biomarker levels were mildly but significantly higher in pre-renal AKI patients than in patients without AKI. In a study by Doi et al. in which 129 out of 337 patients who were admitted to the intensive care unit (ICU) were diagnosed with AKI and in which transient AKI (pre-renal AKI) was defined as the recovery of the serum creatinine $(\mathrm{sCr})$ to within $0.3 \mathrm{mg} / \mathrm{dL}$ above baseline within $48 \mathrm{~h}, 51$ patients were diagnosed with transient AKI [107]. Upon ICU admission, transient AKI patients' levels of urinary NGAL, urinary L-FABP, NAG, and urinary albumin were mildly but significantly higher than those of non-AKI patients. Nejat et al. compared the urinary biomarkers upon ICU admission of 285 non-AKI patients, 61 pre-renal AKI patients (with pre-renal AKI defined as a $\mathrm{FENa}<1.0 \%$ and recovery of the $\mathrm{sCr}$ levels within $48 \mathrm{~h}$ ), and 114 renal AKI patients [155]. The median urinary NGAL levels of non-AKI patients and of pre-renal AKI patients were $7.7 \mu \mathrm{g} / \mathrm{mmolCr}(3.3-35 \mu \mathrm{g} / \mathrm{mmolCr})$ and $14 \mu \mathrm{g} / \mathrm{mmolCr}(6.5-56 \mu \mathrm{g} / \mathrm{mmolCr})$, respectively; thus, pre-renal AKI patients showed a tendency to have a mildly higher urinary NGAL level $(p=0.052)$. In addition, the median urinary NGAL level of renal AKI patients was $44 \mu \mathrm{g} / \mathrm{mmolCr}(16-345 \mu \mathrm{g} / \mathrm{mmolCr})$, which was significantly higher than that of pre-renal AKI patients. The median urinary cystatin $C$ levels of non-AKI patients and pre-renal AKI patients were $0.026 \mathrm{mg} /$ mmolCr $(0.010-0.12 \mathrm{mg} / \mathrm{mmolCr})$ and $0.054 \mathrm{mg} /$ mmolCr $(0.017-0.53 \mathrm{mg} / \mathrm{mmolCr})$, respectively; thus, the urinary cystatin $\mathrm{C}$ was significantly higher in pre-renal AKI patients than in non-AKI patients. The median urinary cystatin $\mathrm{C}$ level of renal AKI patients was $0.21 \mathrm{mg} / \mathrm{mmolCr}(0.05-1.9 \mathrm{mg} / \mathrm{mmolCr})$, which was significantly higher than that of pre-renal AKI patients.

As described above, the urinary NGAL is mildly elevated in pre-renal AKI patients and highly elevated in renal AKI patients, which suggests that the urinary NGAL is potentially useful for the differentiation of pre-renal from renal AKI. However, the measurement points, cutoff values, and the need for urine creatinine correction have not yet been determined; these issues must be considered in the future. Therefore, pre-renal and renal AKI cannot be differentiated based on the urinary NGAL alone; hence, we recommend a comprehensive assessment that also incorporates other laboratory and physical findings.

\section{Literature review}

PubMed was searched for relevant studies published up to November 2015, and papers related to the present CQ were abstracted from the search results.
Search query: "acute kidney injury"[MeSH Terms] OR acute kidney failure[tw] OR acute renal failure[tw] OR acute kidney injury[tw] OR acute kidney injuries[tw] OR acute kidney injury[tw] OR acute kidney injury[tw] OR acute renal injuries[tw] OR acute renal injury[tw] OR acute kidney insufficiencies[tw] OR acute kidney insufficiency[tw] OR acute renal insufficiencies[tw] OR acute renal insufficiency[tw] OR acute tubular necrosis[tw] OR ARI[tw] OR AKI[tw] OR ARF[tw] OR AKF[tw] OR ATN[tw] AND NGAL[tw] OR neutrophil gelatinase-associated lipocalin[tw] OR L-FABP[tw] OR liver-type fatty acid-binding protein[tw] OR NAG[tw] OR N-acetyl$\beta$-D-glucosaminidase[tw]) AND ("pre-renal" $[\mathrm{tw}] \mathrm{OR}$ "pre-renal azotaemia" [tw] OR prerenal[tw]) NOT (child[tw] OR children[tw] OR infant $[\mathrm{tw}]$ OR pediatrics[tw]).

\section{CQ6-1: Is low-dose atrial natriuretic peptide recommended for prevention or treatment of AKI?}

Recommendation: Although low-dose atrial natriuretic peptide has been suggested to be useful for prevention of AKI, relevant reports remain insufficient. Evidence of low-dose atrial natriuretic peptide for the treatment of AKI is limited.

Strength of recommendation: Not graded

Quality of evidence: D

\section{Summary of evidence}

Since atrial natriuretic peptide (ANP) preparation "carperitide" is covered by health insurance for the treatment of congestive heart failure in Japan, we only investigated randomized controlled trials (RCTs) of ANP in which carperitide was used. A 2009 Cochrane review [156] suggested that low-dose ANP may reduce the frequency of renal replacement therapy (RRT) in the setting of AKI prevention. However, the 2012 KDIGO Clinical Practice Guideline for AKI [3] and a 2013 Cochrane review [157] carefully assessed individual pieces of evidence and lead to a revised conclusion that there is insufficient evidence to declare that low-dose ANP is effective for the treatment or prevention of AKI. Regarding 2009 and 2011 reports on cardiovascular surgery which focused on AKI prevention [158, 159], doubts were raised concerning issues such as randomization and blinding methods. In a 2011 paper, administration of low-dose ANP resulted in a significantly reduced rate of RRT after 1 year [159]. Since 2011, there have been two new RCTs related to AKI prevention; however, due to few numbers of patients, we judged these RCTs lack sufficient statistical power. Currently, there is no strong evidence indicating that low-dose ANP is ineffective for the prevention or treatment of AKI, but rather the evidence that does indicate its effectiveness is of insufficient quality. 


\section{Commentary}

Atriuretic peptide (ANP) is a circulating hormone that was discovered in Japan. Along with brain natriuretic peptide (BNP) and C-type natriuretic peptide (CNP), they make up the natriuretic peptide family [160-162]. In healthy conditions, ANP is produced from the atria; however, in heart failure, the production and secretion of ANP from both the atria and ventricles are enhanced [162, 163]. ANP possesses multiple independent modes of actions, including vasodilation, inhibition of sodium reabsorption, inhibition of water reabsorption, elevation of glomerular filtration rate via afferent arteriole dilation and efferent arteriole constriction, reduction of renin activity, angiotensin II concentration, aldosterone concentration in the blood, and sympathetic nerve inhibition [164]. Combined together, continuous infusion of ANP or BNP on laboratory animals and humans exerts a powerful natriuretic effect [165]. Therefore, in the prevention or treatment of AKI, ANP is expected to elicit renoprotective effect through diuresis and increase of glomerular filtration rate. Many clinical studies have been carried out in this context. However, administration of high-dose ANP reduces systemic blood pressure, thereby potentially canceling out the abovementioned renoprotective effect. Therefore, it is crucial to identify the optimal dose of ANP for the achievement of renoprotective effect. Based on the 2012 KDIGO Clinical Practice Guideline for AKI, the present guideline defined low-dose ANP as $\leq 50 \mathrm{ng} / \mathrm{kg} / \mathrm{min}$ and high-dose ANP as $\geq 100 \mathrm{ng} / \mathrm{kg} / \mathrm{min}$.

As to the assessment of the therapeutic effect of ANP after the development of AKI, there are two large-scale randomized controlled trials (RCTs) in which more than 200 participants were assigned into two arms. In both RCTs, high-dose ANP (200 ng/ $/ \mathrm{kg} / \mathrm{min}, 24 \mathrm{~h})$ failed to reduce the incidence of RRT [166, 167]. In a later small-scale RCT using low-dose ANP (50 ng/kg/min, mean $127 \mathrm{~h}$ ), the ANP group exhibited a significant reduction in the frequency of RRT as compared with the placebo group [168]. There have been no subsequent RCTs investigating the therapeutic effects of low-dose ANP for AKI. Therefore, the present guideline could not offer a definitive recommendation.

On the other hand, concerning the prevention of AKI by ANP, 13 RCTs were found (excluding AKI from contrast-induced nephropathy); all of these were Japanese clinical trials which used low-dose ANP. In most of them, the serum creatinine $(\mathrm{sCr})$ values became significantly lower in the ANP group than in the control group. However, based on a strict application of the AKI diagnostic criteria from the 2012 KDIGO Clinical Practice Guideline for AKI [3], we found no studies in which the ANP group demonstrated a significant reduction in the incidence of AKI.
In a 2009 Cochrane review by Nigwekar et al. [156], low-dose ANP was reported to potentially reduce the need for RRT during prevention of AKI in major surgery, particularly cardiovascular surgery. However, the administration of high-dose ANP for AKI treatment was shown to increase the frequency of adverse events such as hypotension and arrhythmia. The 2012 KDIGO Clinical Practice Guideline for AKI and a 2013 Cochrane review by Zacharias et al. [157] judged that the numbers of patients, the details of the randomization and blinding, and the rigor of the endpoint definitions were insufficient in previous studies. Consequently, the effectiveness of low-dose ANP for the prevention of AKI was deemed inconclusive.

Although RCTs conducted in Japan have suggested that ANP is useful for the prevention of AKI, the quality of the research methods used was debatable. Therefore, we conclude that evidence for the effectiveness of low-dose ANP both for the prevention and treatment of AKI is insufficient, making a definitive recommendation impossible. The ANP preparations used in RCTs in Japan and in the West are carperitide (product name: Hamp ${ }^{\circ}$ ) and anaritide, respectively. Although carperitide has been available in Japan for the treatment of congestive heart failure since 1995, its use for the prevention or treatment of AKI is not covered by health insurance. Urodilatin (product name: Ularitide ${ }^{\circ}$ ) is an ANP-related hormone with four amino acid residues added to the $\mathrm{N}$-terminus of ANP; it is produced in the distal nephron [169]. Clinical trials for ularitide have been conducted outside of Japan [170], and we did not mention it in the present guideline.

\section{Literature review}

Searches were conducted for relevant studies published between January 2008 and August 2015. The literature published prior to 2008 was referenced from a 2009 Cochrane review by Nigwekar et al. [156]. All RCTs related to contrast-induced nephropathy were excluded.

\section{CQ6-2: Are loop diuretics recommended for the prevention and treatment of AKI?}

Recommendation: We do not recommend loop diuretics for the prevention of AKI. We also suggest that loop diuretics should not be administered for the treatment of AKI, except to correct fluid overload.

\section{Prevention:}

Strength of recommendation: 1

Quality of evidence: B

Treatment:

Strength of recommendation: 2

Quality of evidence: C 


\section{Summary of evidence}

Previous guidelines and systematic reviews do not recommend the use of loop diuretics for the prevention or treatment of AKI. There have been no new RCTs to contradict the results of previous clinical trials on loop diuretics for AKI.

\section{Commentary}

Loop diuretics inhibit the sodium reabsorption and exert a diuretic effect by inhibiting the $\mathrm{Na}-\mathrm{K}-2 \mathrm{Cl}$ cotransporter in the thick ascending limb of the loop of Henle. Due to their theoretical effectiveness against acute kidney injury (AKI), clinical trials involving loop diuretics have long been performed. For example, by ensuring a diuretic effect, loop diuretics can prevent the tubular obstruction induced by cell shedding; in addition, they increase the medullary oxygenation and the renal medullary blood flow.

Three randomized controlled trials (RCTs) have compared the use of loop diuretics to that of a placebo or to standard therapy for the prevention of AKI [171-173]. In a meta-analysis by Ho and Power, loop diuretics failed to yield a significant improvement in the in-hospital mortality or in the percentage of patients who required renal replacement therapy (RRT) [174]. Moreover, although different RCTs have defined AKI differently, none has yet demonstrated a statistically significant reduction in the incidence of AKI in a loop diuretics group. In fact, in an RCT by Lassnigg et al., the loop diuretics group showed an increased incidence of renal dysfunction (14.6 vs $0 \%, p<0.01)$ [172]. Based on the above, the present guideline does not recommend the use of loop diuretics for AKI prevention.

Seven RCTs have also compared the use of loop diuretics to that of a placebo or to standard therapy for the treatment of existing AKI [175-181]. In the abovementioned meta-analysis, the loop diuretics group did not demonstrate a significant improvement in the in-hospital mortality or the percentage of patients who required RRT [174]. Although different RCTs have used different definitions of recovery from renal dysfunction, no RCT to date has demonstrated a significant increase in the percentage of patients who recovered from renal dysfunction in the loop diuretics group. Among the abovementioned seven RCTs, two limited their subjects to AKI patients who underwent RRT; in both of these, the loop diuretics group did not demonstrate a significant reduction in the duration of the RRT or in the early recovery from renal dysfunction [180, 181]. In addition, one meta-analysis showed that high-dose furosemide, which is often used to treat AKI, significantly increased symptoms such as tinnitus and temporary deafness (as compared with their incidence in control groups) [182]. Based on the above, the present guideline does not recommend the use of loop diuretics for AKI treatment.

On the other hand, in AKI with a reduced urine output, loop diuretics may help to correct the fluid overload and to improve any electrolyte imbalance (such as hyperkalemia). However, there are currently no RCTs in which loop diuretics were administered specifically to treat AKI with these sorts of clinical manifestations-hence the above suggestion. The guidelines published by the KDIGO and NICE (National Institute for Health and Clinical Excellence) do not exclude the use of loop diuretics for the correction of fluid overload.

As for diuretics other than loop diuretics, RCTs have also examined mannitol for the prevention of AKI. In a meta-analysis by Yang et al., mannitol did not demonstrate evident effectiveness for the prevention of AKI [183]. In subsequent RCTs, the mannitol groups also failed to demonstrate significant improvement in their RRT initiation rates or in-hospital mortality $[184,185]$.

\section{Literature review}

PubMed was searched for relevant studies published between January 2012 and April 2015, and papers related to the present CQ were identified from the search results. The literature published before January 2012 was referenced from the KDIGO Clinical Practice Guideline for AKI.

\section{CQ6-3: Is low-dose dopamine recommended to prevent and treat AKI?}

Recommendation: We recommend not using low-dose dopamine to prevent or treat AKI.

Strength of recommendation: 1

Quality of evidence: A

\section{Summary of evidence}

The KDIGO Clinical Practice Guideline for AKI suggests not to use low-dose dopamine to prevent or treat AKI. Since the publication of the KDIGO guideline, the efficacy of low-dose dopamine for the prevention of AKI has been examined in five RCTs. None of them have found low-dose dopamine to be effective.

\section{Commentary}

Dopamine has been widely used to treat severely ill patients especially before 2000 . The administration of dopamine, particularly low-dose $(1-3 \mu \mathrm{g} / \mathrm{kg} / \mathrm{min})$, to healthy individuals is considered to bring increases in renal vasodilation, in natriuresis, and in the glomerular filtration rate (GFR); therefore, low-dose dopamine had been anticipated to have a renoprotective effect. However, many of the clinical studies on dopamine have been found to be of poor quality due to a variety of issues, including small numbers 
of patients, unsuitable randomization, insufficient statistical powers, and unsuitable outcomes related to the clinical utility. Furthermore, due to the negative results in several randomized controlled trials (RCTs) that applied appropriate statistical powers and sample sizes, the use of dopamine is less commonly recommended today [186]. In addition, the renal vasodilation effect observed in healthy individuals has been found not to occur in acute kidney injury (AKI) patients [187].

However, there is only limited evidence of harm caused by the use of low-dose dopamine to prevent or treat AKI. Although a 2005 meta-analysis by Friedrich et al. [188] did not find low-dose dopamine to significantly increase the incidence of adverse effects, there are many literatures related to the adverse effects of dopamine. The potential adverse effects of dopamine include tachycardia, myocardial ischemia, a reduced intestinal blood flow, hypopituitarism, and the inhibition of the $\mathrm{T}$ cell function.

Friedrich et al. conducted a meta-analysis of studies in which low-dose dopamine was used to treat or prevent AKI [188]. Their analysis of 61 randomized and semi-randomized trials determined that low-dose dopamine did not prolong survival, reduce the rate of dialysis initiation, or improve the renal function; in addition, the urine output was found to be only improved on the day the dopamine treatment was initiated. Based on the absence of positive studies about the use of dopamine to prevent and treat AKI, and in consideration of the information about the previously described adverse effects of dopamine, the 2012 KDIGO Clinical Practice Guideline for AKI recommends that low-dose dopamine should not be used to prevent or treat AKI (1A).

For the present $C Q$, we searched the literature to retrieve new evidence that has emerged since the publication of the KDIGO guideline. The literature review and assessment of the abstracts revealed five trials that potentially contained new evidence not included in the existing meta-analyses [189-193]. The subjects were heart failure patients in three trials, laparoscopic surgery patients in one trial, and severe obstructive jaundice patients in one trial. In the three trials involving heart failure patients, dopamine failed to improve the outcomes. The trials involving laparoscopic surgery patients and severe obstructive jaundice patients did not examine any clinically useful outcomes.

Based on the above-described KDIGO guideline recommendation and on the fact that no subsequent trials have demonstrated low-dose dopamine to be effective in the prevention or treatment of AKI, we offer the same quality of evidence and strength of recommendation as the KDIGO guideline.

\section{Literature review}

PubMed was searched for relevant studies published between December 24, 2009, and December 22, 2014, using "dopamine", "AKI", and "RCT" as search terms.

Search query: (("dopamine"[MeSH Terms] OR "dopamine"[All Fields]) AND (("kidney"[MeSH Terms] OR "kidney"[All Fields]) OR renal[All Fields]) AND low[All Fields]) AND (Randomized Controlled Trial[ptyp] AND “2009/12/24”[PDAT]:“2014/12/22”[PDAT] AND "humans"[MeSH Terms]).

\section{CQ6-4: What nutritional support is recommended for AKI treatment?}

Recommendation: We suggest that the administration of calorie and protein as nutritional support for AKI treatment be tailored to the severity and the underlying disease. For severe AKI, we recommend enteral nutrition whenever possible. Unless there is an advanced electrolyte imbalance, strict protein restriction is not necessary.

Strength of recommendation: 2

Quality of evidence: D

\section{Summary of evidence}

Since the publication of the KDIGO guideline, there have been no RCTs regarding nutritional support with subjects limited to AKI patients. The KDIGO guideline recommends a calorie intake of $20-30 \mathrm{kcal} / \mathrm{kg} /$ day for AKI patients of any stage. The desired amounts of protein administration are $0.8-1.0 \mathrm{~g} / \mathrm{kg} /$ day in hypermetabolic AKI patients who do not require dialysis and $1.7 \mathrm{~g} /$ $\mathrm{kg} /$ day in hypermetabolic patients undergoing CRRT; when possible, nutrition through the enteral route is preferred.

\section{Commentary}

Acute kidney injury (AKI) is the form of organ failure to which severely ill patients are most susceptible. Thus, the metabolism is greatly affected by the primary disease, the malnutrition severity, the presence of comorbid organ failure, and the performance of renal replacement therapy (RRT). Therefore, the target levels of the calorie intake and the necessary protein should ideally be tailored to the individual pathology; however, the specific efficacy of nutritional support for AKI has not been demonstrated [194, 195].

Enteral nutrition is considered to be more effective than intravenous nutrition for intestinal mucosa maintenance, bacterial translocation, and the prevention of organ dysfunction. In the meta-analyses of studies involving critically ill patients (including AKI patients), the initiation of enteral nutrition within $24 \mathrm{~h}$ after intensive care unit (ICU) admission was shown to significantly reduce the mortality and the incidence of infectious complications and to shorten the hospital stay lengths; 
however, negative results have also been reported [196-199]. In order to provide sufficient calorie, amino acids, and protein, a combination of intravenous and enteral nutrition is sometimes considered. A group of patients who only received vitamins and trace elements through enteral nutrition for the first 7 days following ICU admission and started intravenous nutrition on day 8 (late-initiation group) demonstrated a significant increase in early discharge (alive) from the ICU/hospital, as well as reductions in the incidence of infections, the number of patients on mechanical ventilation for $>$ 2 days, the duration of RRT, and the health care costs [200].

The target calorie provision levels can be determined with a simple body mass conversion equation $(25 \mathrm{kcal} /$ $\mathrm{kg} /$ day), a calorie consumption prediction equation (Harris-Benedict equation), or the measurement of the energy consumption with an indirect calorimeter. In the first 7 days of sepsis treatment of patients who are not yet critically ill and are not malnourished, energy replenishment by enteral nutrition is recommended; however, supplemental intravenous nutrition to reach the target energy level is not recommended, as it can affect the prognosis adversely [201]. The preferable method to reach the target energy level is to start with a small amount of energy and to increase it gradually based on factors such as the presence of aspiration and/or regurgitation of gastric contents and diarrhea. In obese patients, it must be noted that the use of the actual body weight in the prediction formula will cause an overestimation of the target energy provision level. Based on the reports that intensive insulin therapy is not useful for mortality reduction, the initiation of insulin control at a blood glucose level $\geq 180 \mathrm{mg} / \mathrm{dL}$ and the setting of a target blood glucose level of $144-180 \mathrm{mg} / \mathrm{dL}$ can be considered valid in severe AKI $[3,202,203]$. At the same time, AKI is reported to occur frequently in acute myocardial infarction patients with a blood glucose level $\geq$ $200 \mathrm{mg} / \mathrm{dL}$ at hospital admission [204, 205].

Protein restriction for the prevention or delay of RRT initiation is not recommended; however, it can be considered when an advanced electrolyte imbalance is present. In non-hypermetabolic AKI patients who do not require RRT, a protein provision of $0.8-1.0 \mathrm{~g} / \mathrm{kg} /$ day is recommended. A protein provision of $<1 \mathrm{~g} / \mathrm{kg} /$ day can induce negative nitrogen balance in patients undergoing RRT, due to the factors such as the loss of approximately $10-15 \mathrm{~g} /$ day of amino acids, especially in continuous renal replacement therapy (CRRT). Therefore, in hypermetabolic patients undergoing CRRT, the KDIGO guideline recommends the administration of $1.7 \mathrm{~g} / \mathrm{kg} /$ day of protein to account for the amount of protein loss, while a protein intake of $2.5 \mathrm{~g} / \mathrm{kg} /$ day is reportedly needed to achieve a positive nitrogen balance
[206, 207]. However, the excess provision of amino acids is indicated to potentially cause azotemia and prolonged RRT [208]. During CRRT, commercially available dialysates and replacement fluids in Japan can cause hypokalemia and hypophosphatemia, and the latter is reported to delay weaning from mechanical ventilation; therefore, appropriate supplementation of potassium and/or phosphate through intravenous or enteral nutrition can be beneficial [194, 209-211]. Outside of Japan, a CRRT dialysate containing $4.0 \mathrm{mEq} / \mathrm{L}$ potassium and $3.7 \mathrm{mg} / \mathrm{dL}$ phosphorus has been developed [212]. However, a switch from CRRT to intermittent renal replacement therapy (IRRT) can easily cause an electrolyte imbalance, thereby calling for a reexamination of the content of the intravenous nutrition or enteral nutrition, including the total fluid volume; in particular, the risk of hyperkalemia must be kept in mind.

There is no clear evidence to recommend nutritional support for mild AKI without fluid overload, dehydration, or an electrolyte imbalance. The International $\mathrm{Nu}$ trition Survey conducted a recent international cross-sectional study on nutritional intervention, in which nine Japanese facilities participated. In this study, the calorie sufficiency rate, protein sufficiency rate, and nutrition provision rate, and in nearly all other parameters in Japanese ICU patients were found to be below the global mean; in addition, the initiation of enteral nutrition was demonstrated to be late. Further research is needed.

\section{Literature review}

PubMed was searched for relevant studies published between January 2012 and April 2016, and papers related to the present CQ were abstracted from the search results. The literature published before January 2012 was referenced from the KDIGO Clinical Practice Guideline for Acute Kidney Injury.

\section{CQ7-1: Should blood purification for AKI be initiated early?}

Recommendation: There is little evidence to support the idea that the early initiation of blood purification for AKI improves the outcomes. The timing of the initiation should be decided in broad consideration of the clinical symptoms and disease conditions.

Strength of recommendation: Not graded

Quality of evidence: C

\section{Summary of evidence}

Out of nine relevant RCTs, three were performed at a single center (two involving patients having undergone cardiac surgery, one involving ICU patients); in all three RCTs, the early initiation of blood purification was associated with a reduced mortality. However, in a 
Table 11 Indications for emergent renal replacement therapy

\author{
Fluid overload resistant to diuretics \\ Hyperkalemia or rapid elevation of serum potassium \\ Uremic symptoms (pericarditis, consciousness disturbance with \\ unknown etiology) \\ Severe metabolic acidosis
}

meta-analysis that included multicenter RCTs, the efficacy of the early initiation of blood purification was not supported.

\section{Commentary}

There has been a consensus that emergent blood purification should be initiated for serious, life-threatening disease states. The KDIGO Clinical Practice Guideline for Acute Kidney Injury (AKI) also states that renal replacement therapy (RRT) should be initiated immediately in the event of potentially fatal changes in the body fluid, electrolyte, and acid-base balance (not graded). The indications for emergent RRT in clinical settings are listed in Table 11.

In observational studies from the 1960s that examined the timing of blood purification initiation, hemodialysis was demonstrated to improve the survival rates not when initiated after AKI had progressed to the point at which symptoms of uremia were present, but when initiated before that point. Since 2000, multiple observational studies have examined the initiation of blood purification when the blood urea nitrogen (BUN) is at a level even lower than that set in the aforementioned observational study. Bagshaw et al. conducted a meta-analysis of 15 clinical studies (including two randomized controlled trials [RCTs], 4 prospective observational studies, and 9 retrospective observational studies) [213]. Although the early initiation of blood purification was found to be associated with favorable outcomes, the timing of the initiation across the different studies examined was significantly heterogeneous; therefore, the early initiation of blood purification could not be definitively recommended.

The effect of the early initiation of blood purification on death has been examined in nine RCTs to date-including some that were not covered in Bagshaw et al.'s meta-analysis. Bouman et al. randomly assigned intensive care unit (ICU) patients presenting with oliguria to early high-volume, early low-volume, and late low-volume hemofiltration, with the survival at day 28 and the recovery of the renal function as primary endpoints; however, the three groups did not demonstrate any differences in their survival at day 28 or their recovery of the renal function [214]. An Indian RCT involving patients who developed AKI in the hospital divided the subjects into two groups: a group in which dialysis was initiated early, when the BUN was $\geq 70 \mathrm{mg} / \mathrm{dL}$ or the serum creatinine $(\mathrm{sCr})$ was $\geq 7 \mathrm{mg} / \mathrm{dL}(n=102)$, and a control group in which dialysis was initiated upon fluid overload, hyperkalemia, or any other indication for emergent dialysis (ultimately, BUN $100.9 \pm 32.6 \mathrm{mg} / \mathrm{dL}$, $\mathrm{sCr} 10.41 \pm 3.3 \mathrm{mg} / \mathrm{dL} ; n=106)$. The comparisons of these two groups revealed no significant differences in the mortality or the recovery of the renal function [215]. In a Canadian open-label pilot trial [216] reported in 2015, patients with volume replete AKI were randomly assigned to an early treatment group $(n=48)$ or a standard treatment group $(n=52)$; the mortality and the recovery of the renal function did not show any significant differences between the two groups. In two single-center RCTs involving post-cardiac surgery patients $[217,218]$, the early initiation of blood purification was associated with a reduced mortality. In a multicenter RCT (the HEROICS study) [219], patients experiencing shock requiring catecholamine support following cardiac surgery were randomly assigned to one of the two groups: an early hemofiltration group $(80 \mathrm{~mL} / \mathrm{kg} / \mathrm{h}$ for $48 \mathrm{~h}$ ) or a standard therapy group that included continuous hemodiafiltration (CHDF) if necessary $(n=112$ for both groups). The two groups did not demonstrate a significant difference in their mortality or recovery of the renal function.

Two additional RCTs involving AKI patients in the ICU were reported in May 2016. In a French multicenter RCT (the AKIKI trial) [220], critically ill patients with severe AKI (stage 3) who required mechanical ventilation or catecholamine infusion were randomly assigned to one of the two groups: an early initiation group $(n=311)$, in which RRT was initiated immediately after randomization, or a delayed initiation group $(n=308)$, in which RRT was not initiated until a criterion such as hyperkalemia, metabolic acidosis, pulmonary edema, a high BUN level (>112 mg/ $\mathrm{dL})$, or oliguria $(>72 \mathrm{~h}$ ) was met. The investigation of the utility of early RRT did not reveal a significant difference in the 60-day mortality of the two groups. In a German single-center RCT (the ELAIN trial) [221], 231 critically ill patients with stage 2 AKI and a plasma NGAL $>150 \mathrm{ng} / \mathrm{mL}$ were randomly assigned to an early group (initiation of RRT immediately after randomization) or a delayed group (initiation of RRT upon progression of AKI to stage 3 or the presence of any absolute indications); the assessment of the 90-day mortality found the latter to be significantly reduced in the early group. However, in the AKIKI trial, the RRT initiation in the early group was late, upon validation of stage $3 \mathrm{AKI}$; this timing corresponded to the delayed group in the ELAIN trial. Moreover, in the ELAIN trial, RRT was initiated as continuous renal replacement therapy (CRRT) and was performed for at least 1 week in all patients; however, in the AKIKI trial, CRRT was performed as the sole method of RRT in only $30 \%$ of patients. Although both of these RCTs examined the early 
initiation of RRT, it must be noted that they differed in their timing of initiation and their treatment modalities.

Among the nine RCTs reported to date, those that recorded the 28-day or 30-day mortality were subjected to a meta-analysis; this meta-analysis did not support the efficacy of early initiation (Fig. 5). Moreover, a meta-analysis that included the AKIKI [220] and ELAIN [221] trials was published [222]. In this analysis of six RCTs, including the AKIKI and ELAIN trials as well as the three previously mentioned RCTs involving non-post-cardiac surgery patients [214-216], the early initiation of blood purification did not show evident efficacy in terms of either mortality (relative risk 0.93 , 95\% confidence interval $0.68-1.26$ ) or recovery of the renal function (relative risk 0.88 , 95\% confidence interval 0.48-1.62).

As of August 2016, the multicenter STARRT RCT [223] is ongoing. In France, a multicenter RCT assessing the utility of early initiation of RRT for septic AKI (corresponding to KDIGO stage 3 ) is ongoing (the IDEAL-ICU study [224]). Both of these RCTs are larger in scale than the previous investigations; therefore, the results obtained may lead to new directions about early initiation.

\section{Literature review}

PubMed was searched for relevant studies published up to August 2015, and papers related to the present CQ were identified from the search results. Important manuscripts published after the search period ([220, 222, 221]) were also incorporated into our recommendation.

\section{CQ7-2: What indicators should be used for discontinuation of the blood purification for AKI?}

Recommendation: Improvements in the clinical data and the urine output can be used to determine the timing of the blood purification discontinuation.

Strength of recommendation: Not graded

Quality of evidence: C

\section{Summary of evidence}

There have been no RCTs relevant to the discontinuation of blood purification. In three observational studies, the urine output and SOFA score were reported to be predictors of the possibility to wean patients from blood purification.

\section{Commentary}

The blood purification for acute kidney injury (AKI) is stopped when the renal function sufficiently recovers. However, very few studies have examined the criteria for discontinuation of the blood purification for AKI. Our literature search found three observational studies that identified predictors of the possibility to wean patients from blood purification to date. Wu et al. retrospectively examined AKI patients $(n=304)$ who required renal replacement therapy (RRT) in the intensive care unit (ICU) after surgery [225]. Of the 94 patients in whom RRT was discontinued, 30 patients needed to resume it within 30 days; the duration of the RRT (odds ratio [OR] $1.06,95 \%$ confidence interval [CI] 1.02-1.10), the SOFA score at cessation (OR 1.44, 95\% CI 1.13-1.83), oliguria (<100 mL over $8 \mathrm{~h}$ ) (OR 4.17, 95\% CI 1.07-16.13), and an age over 65 years (OR 6.35, 95\% CI 1.61-24.99) were identified as predictors of discontinuation failure. Kawarazaki et al. retrospectively examined AKI patients $(n=343)$ in Japanese ICUs who required continuous renal replacement therapy (CRRT) [226]. The comparison of an early recovery group (those who could discontinue CRRT within $48 \mathrm{~h}$ of initiation; $n=52$ ) and a control group that excepted patients who died early $(n=239)$ revealed that the urine output upon CRRT initiation $(\mathrm{mL} / \mathrm{h})(\mathrm{OR} 1.02,95 \%$ CI 1.01-1.03), the SOFA score upon CRRT initiation (OR 0.87, 95\% CI 0.780.96), and the time from ICU admission to CRRT initiation (in days) (OR 0.65, 95\% CI 0.43-0.87) were significantly associated with early weaning from CRRT. It is of note that the urine output and SOFA score data were collected upon CRRT initiation, and some patients who ceased the CRRT within $48 \mathrm{~h}$ may not have required blood purification; therefore, these results should be interpreted with caution.

The urine output is perhaps the most clinically emphasized predictor; one useful reference is a sub-analysis of the BEST study $(n=1006)$, in which Uchino et al. examined AKI patients in ICUs in 23 countries [227]. Those who did not require RRT for at least 7 days after the initial discontinuation were defined as the success

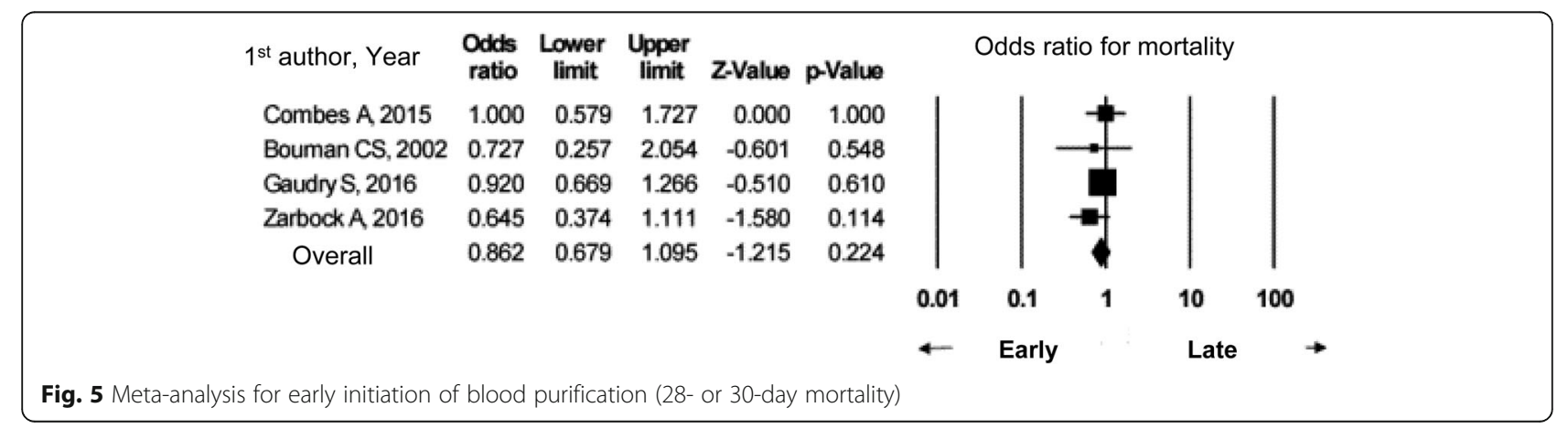


group ( $n=313)$, while those who had to resume RRT within 7 days after the initial discontinuation were defined as the repeat-RRT group $(n=216)$. Comparisons of the two groups revealed that the urine output was the most useful predictor of RRT weaning; the cutoff values for the use and the non-use of diuretics were $2330 \mathrm{~mL} /$ day (approx. $100 \mathrm{~mL} / \mathrm{h}$ ) and $436 \mathrm{~mL} /$ day (approx. $20 \mathrm{~mL} / \mathrm{h}$ ), respectively.

In the previously cited sub-analysis of the BEST study, the serum creatinine $(\mathrm{sCr})$ was also reported to be a significant predictor of weaning (OR 0.996, 95\% CI 0.9940.998). Creatinine is produced from creatine in the muscle tissue and is released into the blood. Both blood purification and the kidneys of the patients remove creatinine. The balance of creatinine between muscle production and elimination by blood purification and kidneys defines the $\mathrm{sCr}$. Therefore, if the $\mathrm{sCr}$ level remains constant for at least 2-3 days, the production and elimination can be considered equal. The phenomenon by which the amount of $\mathrm{sCr}$ remains constant for several days before suddenly decreasing sharply without changing blood purification doses-called "spontaneous fall"-indicates that the renal function has recovered. In the VA/NIH ATN study [228] that examined the association between the dialysis doses and the AKI outcomes, the recovery of the renal function was defined as a urine output $>30 \mathrm{~mL} / \mathrm{h}$ in $6 \mathrm{~h}$ of collection or a spontaneous fall in the $\mathrm{sCr}$. The following protocol was adopted: if the creatinine clearance in the 6-h urine collection was $>20 \mathrm{~mL} / \mathrm{min}$, the CRRT was discontinued; if the creatinine clearance was $<12 \mathrm{~mL} / \mathrm{min}$, the CRRT was continued; if the creatinine clearance was between 12 and $20 \mathrm{~mL} / \mathrm{min}$, the decision to continue or discontinue the CRRT was left to the clinician.

However, in AKI and advanced chronic kidney disease (CKD), creatinine not only undergoes glomerular filtration but is also excreted into the urine due to re-secretion from the renal tubule; consequently, the creatinine clearance is greater than the actual glomerular filtration rate (GFR). In addition, if the $\mathrm{sCr}$ continues to decrease in 6-h urine collection, the $\mathrm{sCr}$ value selected for use in the GFR calculation may result in an overestimation or underestimation of the GFR. At the AKI recovery stage, in which the renal function fluctuates dynamically, the $\mathrm{sCr}$ and creatinine clearance are markedly unreliable; however, due to the absence of other appropriate endpoints, the $\mathrm{sCr}$ may be an acceptable basis upon which to determine whether to discontinue the blood purification, with the above-described background considered.

\section{Literature review}

PubMed was searched for relevant studies published up to August 2015, and papers related to the present CQ were identified from the search results.

\section{CQ7-3: How should the blood purification dose be determined for AKI?}

Recommendation: There is no evidence allowing for the recommendation of an optimal blood purification dose. The dose must be determined individually by considering disease conditions.

Strength of recommendation: 2

Quality of evidence: B

\section{Summary of evidence}

Increasing the dose of blood purification for AKI to higher than the level recommended as the international standard $(20-25 \mathrm{~mL} / \mathrm{kg} / \mathrm{h})$ has not been reported to improve the AKI outcomes. No RCTs have compared the blood purification dose covered by health insurance in Japan $(10-15 \mathrm{~mL} / \mathrm{kg} / \mathrm{h})$ to that recommended internationally; only two observational studies have evaluated this issue, and neither observed a significant difference in mortality. Thus, there is no definitive evidence to support the need to change the dose used in Japan to that recommended as the international standard.

\section{Commentary}

The appropriate dose of renal replacement therapy (RRT) for acute kidney injury (AKI) has been investigated so far. Several studies have reported that increased doses do not lead to improved outcomes [214, 228-230]; in addition, there is insufficient evidence to determine an optimal dose.

The dose of continuous renal replacement therapy (CRRT) for AKI was firstly examined by a 2000 study by Ronco et al. [231]. A total of 425 AKI patients who required continuous hemofiltration (CHF) were randomly assigned a filtration flow rate (QF) of 20,35 , or $45 \mathrm{~mL} / \mathrm{kg} /$ $\mathrm{h}$. The comparisons of the three groups revealed respective survival rates of 41,57 , and $58 \%$; the $20 \mathrm{~mL} / \mathrm{kg} / \mathrm{h}$ group demonstrated a significantly lower survival rate than the other two groups, while there was no significant difference between the $35 \mathrm{~mL} / \mathrm{kg} / \mathrm{h}$ group and the $45 \mathrm{~mL} / \mathrm{kg} / \mathrm{h}$ group. Since then, two multicenter, large-scale randomized controlled trials (RCTs) have been reported (in 2008 and 2009, respectively) [228, 230]; unlike in the trial reported by Ronco et al., these two RCTs found that increased doses of RRT for AKI did not improve the outcomes. In the ATN study [228], 1124 AKI patients who required RRT were randomly assigned to a standard therapy group or an intensive therapy group, and the two groups' mortality and recovery of the renal function were compared. In the standard therapy group, hemodynamically stable patients underwent hemodialysis (HD) three times a week, while hemodynamically unstable patients either underwent continuous hemodiafiltration (CHDF) at a rate of $25 \mathrm{~mL} / \mathrm{kg} / \mathrm{h}$ or received sustained low-efficiency dialysis (SLED) three times a week. In the intensive therapy group, 
hemodynamically stable patients underwent HD six times a week, while hemodynamically unstable patients either underwent CHDF at a rate of $35 \mathrm{~mL} / \mathrm{kg} /$ $h$ or received SLED six times a week. The comparisons revealed no significant differences in the mortality or recovery of the renal function of the two groups. In the RENAL study [230], 1508 AKI patients were randomly assigned to an intensive therapy group (35 $\mathrm{mL} / \mathrm{kg} / \mathrm{h} \mathrm{CHDF}$ ) or a standard therapy group (25 mL/kg/h CHDF), and the two groups' mortality and recovery of the renal function were compared. Likewise, the two groups in this study did not demonstrate any significant differences in their mortality or recovery of the renal function. Based on the results of two multicenter, large-scale RCTs, the recent KDIGO Clinical Practice Guideline for AKI [3] recommends a CRRT dose of $20-25 \mathrm{~mL} / \mathrm{kg} / \mathrm{h}$. However, both the ATN and RENAL studies examined AKI collectively with a wide variety of causes, including ischemia, nephrotoxic substances, and sepsis; to date, there have been very few studies of the optimal doses in function of the underlying disease. Regarding septic AKI, four RCTs [232-235] have compared the CRRT outcomes from doses of $35-45 \mathrm{~mL} / \mathrm{kg} / \mathrm{h}$ and larger doses $(65-100 \mathrm{~mL} / \mathrm{kg} / \mathrm{h})$; in these RCTs, increased doses were not found to improve the outcomes. Based on the above, there is currently no definitive evidence allowing for the recommendation of optimal doses according to the underlying disease. However, in the event of acute hyperkalemia-such as in tumor lysis syndrome-the blood purification doses must be temporarily increased or otherwise tailored to the individual pathologies.

The common dose of CRRT for AKI in Japan (10$15 \mathrm{~mL} / \mathrm{kg} / \mathrm{h}$ ) is generally smaller than the recommended dose outside of Japan $(20-25 \mathrm{~mL} / \mathrm{kg} / \mathrm{h})$. This seems to be because Japanese health insurance only covers a dialysis dose of approximately $15 \mathrm{~L} /$ day. No RCTs have compared the standard Japanese dialysis dose of $10-15 \mathrm{~mL} / \mathrm{kg} / \mathrm{h}$ with the recommended international dose of $20-25 \mathrm{~mL} / \mathrm{kg} / \mathrm{h}$; however, two retrospective observational studies [236, 237] have concluded that the standard Japanese dose did not lead to worse outcomes. As mentioned above, although Ronco et al. reported an optimal blood purification dose of $35 \mathrm{~mL} / \mathrm{kg} / \mathrm{h}$, this was controverted by two subsequent large-scale RCTs. However, there is currently insufficient evidence to determine that 20$25 \mathrm{~mL} / \mathrm{kg} / \mathrm{h}$ is an optimal dose, and the standard Japanese dose must be examined as well. Moreover, while it is unknown whether the reduction of doses to below the standard Japanese dose of $10-15 \mathrm{~mL} / \mathrm{kg} /$ $\mathrm{h}$ would worsen the outcomes, we would like to address that there is no evidence to recommend the reduction of dialysis doses.
The appropriate doses of HD for AKI have been examined in three RCTs [214, 228, 238]. In those, the different groups demonstrated no significant differences in their mortality or recovery of the renal function. For intermittent renal replacement therapy (IRRT) or extended RRT, the KDIGO Clinical Practice Guideline for AKI recommends a weekly standardized dialysis dose $(\mathrm{Kt} / \mathrm{V})$ of 3.9. However, there is insufficient evidence to establish this as the optimal hemofiltration dose for AKI. An RCT that compared HD and predilution online HF rather than HD doses was reported in 2012 [239]. The mean volume of infusate in predilution online HF was $81 \mathrm{~L}$; the HF and HD groups did not demonstrate significant differences in their mortality or recovery of the renal function. In a prospective study by Schiffl et al. that compared a daily HD group with an alternate-day HD group [238], the daily HD group demonstrated a significantly lower mortality and a significantly earlier recovery of the renal function. However, several issues have been raised with this study: the HD doses were extremely low, and the randomization was inadequate. The Hannover Dialysis Outcomes Study randomly assigned 156 AKI patients to a standard dialysis group and an intensified dialysis group and compared the mortality and the recovery of the renal function in the two groups [240]. The standard dialysis was dosed to maintain a blood urea nitrogen (BUN) level of $120-150 \mathrm{mg} / \mathrm{dL}$, while the intensified dialysis was dosed to achieve a target BUN level of $<90 \mathrm{mg} / \mathrm{dL}$. However, the two groups did not demonstrate significant differences in their mortality or recovery of the renal function.

\section{Literature review}

PubMed was searched for relevant studies published up to July 2015, and papers related to the present CQ were identified from the search results.

\section{CQ7-4: Should blood purification for AKI be performed continuously or intermittently?}

Recommendation: In hemodynamically stable patients, blood purification may be performed either continuously or intermittently. In hemodynamically unstable patients, continuous blood purification is preferable.

Hemodynamically stable patients:

Strength of recommendation: 2

Quality of evidence: B

Hemodynamically unstable patients:

Strength of recommendation: Not graded

Quality of evidence: C

\section{Summary of evidence}

Several RCTs and meta-analyses have compared CRRT with IRRT, but none have demonstrated differences in mortality. It must be noted that some of these RCTs 
excluded hemodynamically unstable patients and that none limited their subjects to hemodynamically unstable patients. In meta-analyses of sustained low-efficiency dialysis (SLED), which combines the advantages of both CRRT and IRRT, comparisons with CRRT revealed no significant difference in the mortality.

\section{Commentary}

The optimal modality of blood purification for acute kidney injury (AKI) has been investigated. The primary continuous and intermittent modalities used in Japan are continuous hemodiafiltration (CHDF) and hemodialysis (HD), respectively [241]. The choice of therapy is generally based on a consideration of various factors, including the patient's hemodynamics and anticoagulation, the facility's equipment, and the staff's experience and manpower. The advantages and disadvantages of these modalities are summarized in Table 12. The greatest advantage of continuous renal replacement therapy (CRRT), which is mostly conducted with CHDF in Japan, is that its effects on the hemodynamics are minimized as it removes the body fluids and solutes gradually. CRRT is also associated with a reduced risk of cerebral edema [242]. However, continuous blood purification not only restrains the patient over a long period of time but also places a great burden on the medical care staff. In addition, the continuous administration of anticoagulants increases the risk of hemorrhage. Intermittent renal replacement therapy (IRRT), which is mostly conducted with HD in Japan, removes the body fluids and solutes quickly, thereby easily affecting the hemodynamics and increasing the risk of cerebral edema. However, in addition to being completed faster, IRRT places a lesser burden on the staff and poses a lower risk of hemorrhage than CRRT. As CRRT and IRRT possess evidently different characteristics, direct comparisons of their utility have been found to be worthless [243].

For the present $\mathrm{CQ}$, we abstracted a total of 15 randomized controlled trials (RCTs) [244-258] and 8 meta-analyses [259-266] that compared the utility of the two modalities of renal replacement therapy (RRT) for

Table 12 Comparison between IRRT and CRRT

\begin{tabular}{lll}
\hline & Advantage & Disadvantage \\
\hline IRRT & $\begin{array}{l}\text { Rapid correction of fluid } \\
\text { and electrolyte } \\
\text { Patient's mobility }\end{array}$ & Hemodynamic intolerance \\
& $\begin{array}{l}\text { Anticoagulation/bleeding } \\
\text { disorders }\end{array}$ & \\
& Low cost & \\
CRRT & Hemodynamic tolerance & $\begin{array}{l}\text { Requirement of continuous } \\
\text { anticoagulation } \\
\end{array}$ \\
& No osmotic cellular shift & High cost \\
& & Workload for medical staff \\
\hline
\end{tabular}

AKI. A primary meta-analysis was reported as part of a Cochrane joint project in 2008 [265]. An analysis of 15 RCTs involving a total of 1550 AKI patients who required RRT revealed no significant differences between CRRT and IRRT in the in-hospital mortality, the ICU mortality, or the discontinuation of RRT in surviving patients. Several other meta-analyses have demonstrated similar results [260-263]. However, in a meta-analysis of 13 studies (including three RCTs) by Kellum et al., while CRRT and IRRT yielded no significant difference in mortality, adjustments for the severity of the illness and the study quality revealed that the risk of death was significantly reduced in CRRT (relative risk [RR] 0.72, 95\% confidence interval [CI] 0.60-0.87) [259]. In Schneider et al.'s meta-analysis of 23 studies (including 7 RCTs) related to the rates of dialysis dependence, although the risk of dialysis dependence was significantly higher in patients undergoing IRRT (RR 1.73, 95\% CI 1.35-2.20), there was no significant difference when the analysis was limited to RCTs [264]. The KDIGO guideline and the Surviving Sepsis Campaign Guideline (SSCG) 2012 [267] considered the same matter as the present $\mathrm{CQ}$ and gave similar recommendations based on the results described above.

It must be noted that two RCTs $[245,254]$ excluded hemodynamically unstable patients. Theoretically and empirically, CRRT has been considered useful, and has therefore been used, for the treatment of hemodynamically unstable patients. No RCT has ever compared CRRT and IRRT in hemodynamically unstable patients. Based on the above, our expert opinion is that CRRT is preferable for hemodynamically unstable patients. However, there is no standard opinion on the degree of instability at which CRRT should be chosen. In addition, many hemodynamically unstable patients are of course critically ill and may have comorbid coagulation disorders, such as disseminated intravascular coagulation (DIC). HD may be more useful in patients with an advanced bleeding tendency, as it can be performed with only a short course of anticoagulant administration. In addition, the selection of a modality must take into account the equipment at the facility, the staff's experience and resources, and a variety of other factors unrelated to the patient. The modality should be decided by a physician with sufficient knowledge and experience of blood purification (i.e., an intensive care specialist, nephrologist, or dialysis physician) according to the patient's disease condition.

Sustained low-efficiency dialysis (SLED), or extended daily dialysis (EDD), uses the lower blood flow and the lower dialysate flow and is performed more frequently than standard HD; it incorporates the advantages of both CRRT and IRRT and is now performed widely. Although no RCTs have compared SLED and intermittent hemodialysis (IHD), the effects of SLED on the 
hemodynamics are reported to be the same as those of CRRT [268]. Recently, Zhang et al. conducted a meta-analysis of 17 studies (including 7 RCTs) that compared EDD and CRRT for AKI [266]. In the RCTs, no significant difference was observed in the mortality of the different groups; however, in the ten observational studies, the risk of death was lower with EDD (RR 0.86, 95\% CI 0.74-1.00). No significant difference in the recovery of the renal function was evidenced by the RCTs or the observational studies. By establishing appropriate implementation conditions, not only CRRT but also EDD may be performed in hemodynamically unstable patients.

\section{Literature review}

PubMed was searched for relevant studies published up to July 2015, and papers related to the present CQ were identified from the search results.

\section{CQ7-5: Should nafamostat mesilate be used as an anticoagulant in blood purification for AKI?}

Recommendation: Nafamostat mesilate may be considered for patients with a high risk of bleeding. For patients with active bleeding, blood purification without the use of anticoagulants may also be considered.

Strength of recommendation: Not graded

Quality of evidence: C

\section{Summary of evidence}

There have only been two RCTs involving the use of nafamostat mesilate as an anticoagulant during blood purification for AKI (nafamostat mesilate vs no anticoagulant). No significant difference was observed in the survival outcomes. Two observational studies that compared the use of nafamostat mesilate and heparin also found no significant difference in the survival outcomes.

\section{Commentary}

The blood coagulates when it comes into contact with anything including artificial materials other than vascular endothelial cells. Therefore, renal replacement therapy (RRT) - which involves extracorporeal circulationusually requires the use of anticoagulants. Patients with severe acute kidney injury (AKI) requiring RRT frequently present with a hemorrhagic complication. Thus, the use of anticoagulants that pose the lowest possible risk of bleeding is required. Japanese health insurance currently covers four types of anticoagulants for RRT: unfractionated heparin, low molecular weight heparin (LMWH), nafamostat mesilate (NM), and argatroban. Citrate is widely used as an anticoagulant outside of Japan. Although it can be used in Japan as well, this is not frequent, since its use as an anticoagulant for RRT is considered off-label. In the BEST kidney study [34], which included an examination of the anticoagulants used in continuous renal replacement therapy (CRRT) for AKI, the most commonly used anticoagulant was unfractionated heparin (42.9\% of patients), followed by no anticoagulant (33.1\%), citrate (9.9\%), NM (6.1\%), and LWMH (4.4\%).

When possible, CRRT without anticoagulation is the safest option for AKI patients, as it does not increase the risk of bleeding; however, the lifespans of the filter and circuit may be shortened, and CRRT cannot be performed without anticoagulation in all patients. In Japan, NM is widely used as an anticoagulant in CRRT for AKI since it has a short half-life and is associated with a relatively lower risk of bleeding than other anticoagulants. However, it has not been approved only in the limited countries. In addition, NM causes adverse effects, including agranulocytosis, hyperkalemia, and anaphylactoid reactions [269-272]. In Japan, unfractionated heparin is the most commonly used anticoagulant for hemodialysis (HD) in chronic dialysis patients. However, due to concerns over the risk of bleeding, it is seldom used as an anticoagulant in CRRT for AKI in Japan. Similarly, although LMWH is also associated with a lower risk of bleeding than unfractionated heparin, the test that indicates anticoagulant action (the anti-Xa assay) is not common. Therefore, LMWH is infrequently used as an anticoagulant in CRRT for AKI [34].

Five studies have examined the use of NM as an anticoagulant in CRRT for AKI [34, 273-276]. Only two of these studies were randomized controlled trials (RCTs) [273, 274], while one was a prospective observational study [34] and two were retrospective studies [275, 276]. Both of the RCTs compared NM with no coagulation, and neither observed a significant difference in the survival outcomes of the groups. In one RCT, the lifetime of the hemofilter was found to be significantly longer with NM than without coagulation. The two groups demonstrated no significant difference in their hemorrhagic complications. A prospective observational study (the BEST kidney study) and a retrospective study by Hwang et al. [276] also observed no significant differences in the survival outcomes. In a retrospective observational study in which continuous hemodiafiltration (CHDF) was performed without anticoagulation in patients at a high risk of bleeding, Baek et al. reported that only NM was used when the hemofilter lifespan was less than $12 \mathrm{~h}$ [275]. The in-hospital mortality was significantly lower in the NM group (anticoagulation-free group vs NM group: 64.6 vs $41.9 \%, p=0.003$ ), while there was no significant difference between the groups in the transfusion volume (anticoagulation-free group vs NM group: 0.7 vs 0.7 units/day). In addition to the five studies cited above, another study in which NM was compared with heparin was recently published in Japan [277]. Despite being a retrospective 
observational study, its analysis featured propensity score-matched cohorts. Although the mortality was not examined, hemorrhagic complications were significantly less frequent in the NM group, while there was no significant difference in the filter lifespan.

Citrate is commonly used as an anticoagulant in CRRT for AKI outside of Japan. Although the safety and efficacy of citrate have been assessed, its use as an anticoagulant in CRRT for AKI is considered off-label in Japan. Although no RCTs have compared citrate with $\mathrm{NM}$, ten RCTs have compared citrate and unfractionated heparin [278-287]; in the six RCTs that examined the survival outcomes [279, 282-284, 286, 287], no significant difference was observed. Eight studies found the filter lifespan to be significantly longer with citrate [278, 279, 282-287], while two observed no significant difference [280, 281]. The frequency of hemorrhagic complications with citrate and unfractionated heparin was found to be either equal or significantly lower with citrate. Five more RCTs examined the use of LMWH as an anticoagulant in CRRT [288-292]; three of them [288, 289, 291] compared LMWH with unfractionated heparin, while two [290, 292] compared LMWH with citrate. Only one of the five RCTs [290] examined the clinical outcomes; in this RCT, the mortality was significantly lower in the citrate group. With regard to bleeding, only one RCT found a significant difference [291]; however, the results related to the filter lifespan varied across studies.

\section{Literature review}

PubMed was searched for relevant studies published up to December 2015, and papers related to the present CQ were identified from the search results. The study by Makino S et al. [277], which was published after the search period, was found through a hand search.

\section{CQ7-6: What membrane material should be chosen for blood purification in AKI?}

Recommendation: There is no evidence for the recommendation of a specific membrane material to improve the outcomes.

Strength of recommendation: 2

Quality of evidence: C

\section{Summary of evidence}

The majority of blood purification filters currently used in Japan are biocompatible high-flux membranes. However, no studies have found the differences in these membranes to affect the AKI outcomes or the recovery of the renal function. For AKI-and particularly septic AKI-blood purification is performed in Japan to improve hypercytokinemia by using the principle of adsorption; however, there is no high-level evidence for the effect of this blood purification method on the outcomes.

\section{Commentary}

The blood purification membrane materials currently used in Japan include cellulose triacetate (CTA), polymethyl methacrylate (PMMA), ethylene vinyl alcohol (EVAL), polysulfone (PS), polyethersulfone (PES), polyarylethersulfone (PAES), and polyester polymer alloy (PEPA). These materials will activate complement system less than cuprophan and other regenerated celluloses that have been used since the 1960s and are considered to be highly biocompatible membranes. Many of these membranes currently in use have been developed as high-flux (HF) membranes, with the goal to remove the $\beta 2$ microglobulin ( $\beta 2 \mathrm{MG}$ ) and other small molecule proteins.

The therapeutic effects of each membrane material have been compared in a few small-scale randomized clinical trials (RCTs) that primarily featured comparisons of regenerated cellulose with synthetic polymeric membranes. These RCTs have also featured comparisons of HF membranes with the low-flux (LF) membranes that were developed prior to the existence of HF membranes. With regard to the present CQ, we found seven relevant RCTs that compared different types of membranes [293-299].

Five of these RCTs primarily compared the effects of the differences in the membranes' biocompatibility, along with the effects of the differences between LF and HF membranes. Schiff et al. [293] examined the recycled cellulose Cuprophan ${ }^{\odot}$ (LF membrane) and the synthetic polymer polyacrylonitrile (PAN; HF membrane) in two groups of 26 postoperative acute kidney injury (AKI) patients each and compared the results of the two groups. In later trials, Jörres et al. [294] compared 76 AKI patients in whom Cuprophan ${ }^{\odot}$ (LF membrane) was used with 84 patients in whom PMMA (LF membrane) was used. Meanwhile, Gastaldello et al. [295] and Albright et al. [296] compared cellulose acetate (LF membrane) with PS (HF membrane)-both of which are improved versions of Cuprophan -in AKI patients. However, none of the above three trials observed differences in the outcomes or in the recovery of the renal function. In 2008, Cochrane reported a meta-analysis of 1100 patients from the five RCTs cited above and from five others (ten RCTs in all); the comparisons of the biocompatible membranes (synthetic polymeric membranes, $n=575$ ) with the bioincompatible membranes (regenerated cellulose membranes, $n=525$ ) revealed no significant differences in the mortality (relative risk $0.93,95 \%$ confidence interval $0.81-1.07$ ) or in the recovery of the renal function $(n=1038$, relative risk $1.09,95 \%$ confidence interval 0.90-1.31) [300]. Although these results are not directly relevant to comparisons of the synthetic polymeric membranes mainly used today, it should be 
acknowledged that no significant differences have been found between synthetic polymeric membranes and the so-called bioincompatible regenerated cellulose membranes.

Jones et al. [297] compared the survival rates for the use of the two synthetic polymeric membranes PAN $(n=97)$ and PS ( $n=100)$ (both HF membranes) in the continuous hemodialysis (CHD) of ventilated patients with AKI. No significant difference was observed (PAN 29\%, PS 27\%). In the 2000s, another trial compared HF and LF membranes made of the same material. Ponkivar et al. [298] compared HF membranes $(n=34)$ and LF membranes $(n=38)$ both made of PS in AKI patients; the two groups' results were similar. Based on the above, none of the blood purification membranes currently used in Japan can produce better AKI treatment outcomes.

The core of the pathophysiology of septic AKI is assumed hypercytokinemia. The improvement of hypercytokinemia may be useful for the improvement of the performance status and of AKI. This has led to attempts at blood purification designed to remove all types of cytokines. Haase et al. [299] conducted a crossover RCT in which ten sepsis patients with AKI classified by the RIFLE criteria as Failure underwent hemodialysis (HF) using a standard HF membrane (with in vivo molecular weight cutoff values of $15-20 \mathrm{kD}$ ) and a membrane with a larger pore size (50-60 kD). Comparisons of the cytokine removal efficiency revealed that the use of a membrane with a larger pore size significantly reduced the blood concentrations of the cytokines IL-6, IL-8, and IL-10 at $4 \mathrm{~h}$ of HD. In Japan, PMMA membranes [301] and AN69ST membranes [302], which are based on the principle of adsorption and are considered to highly efficient at removing cytokines, have been used in attempts at blood purification. Since 2014, AN69ST membranes have been covered by health insurance in Japan for patients with severe sepsis and septic shock. However, there is no high-level evidence of the clinical efficacy of membranes with large pore sizes or of adsorption membranes. Therefore, in regard to blood purification for the treatment of sepsis, the KDIGO guideline states, "Until further evidence becomes available, the use of RRT to treat sepsis should be considered experimental." The collection of further evidence is anticipated.

\section{Literature review}

PubMed was searched for relevant studies published up to July 2015 , and papers related to the present CQ were identified from the search results.

\section{CQ8: Do AKI patients require long-term follow- up?}

Recommendation: The long-term outcomes of AKI are poor. Therefore, we suggest confirming patients' condition at approximately 3 months later and conducting long-term follow-up in accordance with their condition.

Strength of recommendation: 2

Quality of evidence: C

\section{Summary of evidence}

At present, there have been no RCTs to examine the long-term outcomes of AKI ( $\geq 12$ months following onset). However, there have been systematic reviews and meta-analyses of observational studies related to the survival outcomes, cerebrovascular and cardiovascular outcomes, and renal outcomes; the most reliable and most recent one is a study by Sawhney et al. Based on the search query used in that systematic review, we further searched the literature for studies related to the survival outcomes, the cardiovascular and cerebral disease outcomes, and the renal outcomes with an extended search period. We then conducted a meta-analysis of the search results, along with a consideration of any new studies related to the survival outcomes and renal outcomes, and of observational studies related to the cerebral and cardiovascular disease outcomes. In the results of our meta-analysis, the long-term outcomes of AKI patients were consistently poor. Moreover, although there have been no meta-analyses of the long-term QOL, some observational studies report that the onset of AKI is associated with a reduced long-term QOL.

\section{Commentary}

The term "acute renal failure" (ARF) was used for the first time in writing by Heberden et al. in 1802 [303]. Although ARF was once considered to be reversible and therefore to have a favorable outcome, Hishida et al. reported that ARF patients have extremely poor survival outcomes and cited multiple organ failure as a crucial underlying factor [304]. There were already multiple definitions of ARF [4]. In order to avoid confusion over the definition of ARF and to define acute syndromes related to the renal function more broadly, the term "acute kidney injury" (AKI) was suggested globally.

As the concept of AKI spread worldwide, many clinical studies on AKI have been performed. These studies have shown that the survival outcomes of AKI are poor [305-308], that its long-term outcomes are also poor [309], and that the stage of AKI in the intensive care unit (ICU) is correlated with the mortality [310]; as a result, perspectives on the outcomes of AKI have been changing. In 2015, Sawhney et al. reported the results of a systematic review that assembled the results of individual studies [311]; the survival outcomes and renal outcomes 1 year after the AKI onset were both shown to be poor. However, other clinically important outcomes such as the cerebral outcomes, the cardiovascular outcomes, and the quality of life (QOL) have not been examined. 


\section{Survival outcomes}

Sawhney et al.'s systematic review reported poor long-term post-AKI survival outcomes [311]. We could not find any subsequent clinical study that yielded different conclusions about the long-term post-AKI survival outcomes.

\section{Cerebral and cardiovascular disease outcomes}

Several meta-analyses of existing observational studies were reported in 2015 [312-314]. Although each of these reports used different subjects and endpoints, they consistently showed that the long-term outcomes of cerebral and cardiovascular diseases in AKI patients are poor. Of note, the subjects in these meta-analyses were limited to all post-cardiovascular surgery patients (post-aortic aneurysm repair [312], post-aortic valve implantation [313], and post-cardiopulmonary bypass [314]).

\section{Renal outcomes}

Sawhney et al.'s systematic review revealed that the long-term post-AKI renal outcomes are poor [311]. We could not find any subsequent manuscript that yielded different conclusions about the long-term post-AKI renal outcomes.

\section{QOL}

As of the end of 2015, there had been few reports and no meta-analysis results relevant to the long-term post-AKI QOL; however, an observational study by Nisula et al. used the EQ5D score [315], and another by Hofhuis et al. used the SF36 [316]. In both studies, the QOL was worse in the AKI group than in the non-AKI group. We did not find any studies on the long-term prognoses of AKI that adopted the ADL or fractures as outcomes.

Based on the above, the long-term post-AKI survival outcomes, cerebral and cardiovascular outcomes, and renal outcomes can all be considered poor. Therefore, patients who develop AKI are thought to require long-term follow-up. Moreover, we recommend conducting an initial follow-up-in which it is recommended to assess the performance status and possible complications-at 3 months in order to evaluate the possible development of chronic kidney disease (CKD). We chose this timing for two reasons: (1) according to the current diagnostic criteria, CKD is defined as a kidney injury that continues for 3 months, meaning that the renal assessment at 3 months post-AKI can be considered valid; (2) we considered consistency with the KDIGO Clinical Practice Guideline for AKI [3].

\section{Literature review}

Based on the search query used by Sawhney et al. in their systematic review [311], we developed a search query to encompass four types of outcomes: survival outcomes, cerebral and cardiovascular outcomes, renal outcomes, and the QOL. PubMed was searched for relevant studies published between January 1, 2005, and April 30, 2015. In regard to the long-term survival outcomes and the long-term renal outcomes, we abstracted results from beyond the subject period used in existing systematic reviews. With regard to the titles and abstracts, we conducted a preliminary review and selected potentially relevant manuscripts; we then conducted a secondary review of these manuscripts (full-text assessments) to identify our final target manuscripts.

\section{CQ9-1: Should the KDIGO diagnostic criteria for AKI be used for children?}

Recommendation:

Age $\geq 3$ months: We suggest using the KDIGO AKI diagnostic criteria to predict the survival outcomes.

Age $<3$ months: We suggest using the modified KDIGO diagnostic criteria for neonates.

Age $\geq 3$ months:

Strength of recommendation: 2

Quality of evidence: C

Age < 3 months:

Strength of recommendation: Not graded

Quality of evidence: D

\section{Summary of evidence \\ Age $\geq 3$ months}

Two single-center retrospective observational studies have assessed the KDIGO diagnostic criteria in sufficiently large cohorts; these studies consistently demonstrated that the KDIGO diagnostic criteria were useful for the prediction of the mortality and of other outcomes.

\section{Age $<3$ months}

Two review papers have examined the diagnosis of AKI in neonates and have commented on the results obtained in a total of 11 observational studies. The modified neonatal KDIGO criteria were suggested with the data of associations with the AKI onset, mortality, and neurological outcomes.

\section{Commentary}

The early diagnosis and treatment of acute kidney injury (AKI) are crucial to the improvement of the outcomes not only in adults but also in children. Several diagnostic criteria have been suggested for children. These have included the Pediatric RIFLE (pRIFLE) (Table 13), AKIN (Table 2), and KDIGO (Table 3) criteria. It is known that the normal serum creatinine $(\mathrm{sCr})$ values change with age (Table 14) [317]. As urine collection is difficult in children, the pRIFLE classification uses a Schwartz 
Table 13 pRIFLE criteria

\begin{tabular}{lll}
\hline & eGFR criteria & Urine output criteria \\
\hline Risk & Decrease in $\mathrm{GGFR} \geq 25 \%$ & $\cup \mathrm{UO}<0.5 \mathrm{~mL} / \mathrm{kg} / \mathrm{h} \times 8 \mathrm{~h}$ \\
Injury & Decrease in eGFR $\geq 50 \%$ & $\cup O<0.5 \mathrm{~mL} / \mathrm{kg} / \mathrm{h} \times 16 \mathrm{~h}$ \\
Failure & Decrease in eGFR $\geq 75 \%$ or eGFR $<35 \mathrm{~mL} / \mathrm{min} / 1.73 \mathrm{~m}^{2}$ & $\cup O<0.3 \mathrm{~mL} / \mathrm{kg} / \mathrm{h} \times 24 \mathrm{~h}$ or anuria $\times 12 \mathrm{~h}$ \\
Loss & Complete loss of kidney function $>4$ weeks & \\
ESKD & End-stage renal disease (dialysis dependent $>3$ months) & \\
\hline
\end{tabular}

eGFR estimated glomerular filtration rate, $s C r$ serum creatinine, ESKD end-stage kidney disease, UO urine output

formula-based [318] calculation of the estimated glomerular filtration rate (eGFR) [319]. Due to the differences between the Japanese and Western body constitutions and renal functions, the assessment of the GFR based on the Schwartz formula is considered unsuitable for Japanese children [320]; therefore, another equation has been proposed to estimate the GFR in Japanese children [321]. The AKIN and KDIGO classifications of AKI are based on the $\mathrm{sCr}$ and on the duration of oliguria/anuria (i.e., a urine output $<0.5 \mathrm{~mL} / \mathrm{kg} / \mathrm{h}$ ). Hereafter, we will describe studies that have compared these multiple diagnostic criteria for pediatric AKI.

Sutherland et al. compared the pRIFLE, AKIN, and KDIGO diagnostic criteria in 14,795 children aged under 18 who were hospitalized for AKI [322]. The AKIN and KDIGO classifications, which both use the $\mathrm{sCr}$ criteria,

Table 14 Pediatric reference ranges of serum creatinine (mg/

\begin{tabular}{|c|c|c|c|c|c|c|}
\hline Age & \multicolumn{2}{|c|}{2.5 percentile } & \multicolumn{2}{|c|}{50 percentile } & \multicolumn{2}{|c|}{97.5 percentile } \\
\hline $3-5$ months & \multicolumn{2}{|l|}{0.14} & \multicolumn{2}{|l|}{0.20} & \multicolumn{2}{|l|}{0.26} \\
\hline $6-8$ months & \multicolumn{2}{|l|}{0.14} & \multicolumn{2}{|l|}{0.22} & \multicolumn{2}{|l|}{0.31} \\
\hline 9-11 months & \multicolumn{2}{|l|}{0.14} & \multicolumn{2}{|l|}{0.22} & \multicolumn{2}{|l|}{0.34} \\
\hline 1 year & \multicolumn{2}{|l|}{0.16} & \multicolumn{2}{|l|}{0.23} & \multicolumn{2}{|l|}{0.32} \\
\hline 2 years & \multicolumn{2}{|l|}{0.17} & \multicolumn{2}{|l|}{0.24} & \multicolumn{2}{|l|}{0.37} \\
\hline 3 years & \multicolumn{2}{|l|}{0.21} & \multicolumn{2}{|l|}{0.27} & \multicolumn{2}{|l|}{0.37} \\
\hline 4 years & \multicolumn{2}{|l|}{0.20} & \multicolumn{2}{|l|}{0.30} & \multicolumn{2}{|l|}{0.40} \\
\hline 5 years & \multicolumn{2}{|l|}{0.25} & \multicolumn{2}{|l|}{0.34} & \multicolumn{2}{|l|}{0.45} \\
\hline 6 years & \multicolumn{2}{|l|}{0.25} & \multicolumn{2}{|l|}{0.34} & \multicolumn{2}{|l|}{0.48} \\
\hline 7 years & \multicolumn{2}{|l|}{0.28} & \multicolumn{2}{|l|}{0.37} & \multicolumn{2}{|l|}{0.49} \\
\hline 8 years & \multicolumn{2}{|l|}{0.29} & \multicolumn{2}{|l|}{0.40} & 0.53 & \\
\hline 9 years & 0.34 & & 0.41 & & 0.51 & \\
\hline 10 years & 0.30 & & 0.41 & & 0.57 & \\
\hline 11 years & 0.35 & & 0.45 & & 0.58 & \\
\hline & Male & Female & Male & Female & Male & Female \\
\hline 12 years & 0.40 & 0.40 & 0.53 & 0.52 & 0.61 & 0.66 \\
\hline 13 years & 0.42 & 0.41 & 0.59 & 0.53 & 0.80 & 0.69 \\
\hline 14 years & 0.54 & 0.46 & 0.65 & 0.58 & 0.96 & 0.71 \\
\hline 15 years & 0.48 & 0.47 & 0.68 & 0.56 & 0.93 & 0.72 \\
\hline 16 years & 0.62 & 0.51 & 0.73 & 0.59 & 0.96 & 0.74 \\
\hline
\end{tabular}

were almost completely in agreement; however, as the eGFR-based pRIFLE classification has a higher incidence for stage 1 than the AKIN or KDIGO classifications, a larger number of patients were diagnosed with mild AKI. In all three classifications, the mortality was higher in patients with AKI than in those without AKI; particularly in the intensive care unit (ICU), the increasing severity of AKI (according to all three classifications) was associated with increased mortality. Selewski et al. used the KDIGO classification to examine the AKI outcomes in a cohort of 2415 patients in pediatric ICUs [323]. In comparison with patients who did not develop AKI, pediatric AKI patients demonstrated a significantly increased length of mechanical ventilation, a longer ICU stay, a longer duration of hospitalization, and a higher mortality rate. In addition, the length of the ICU stay was proportional to the worsening of the KDIGO AKI stages. These two single-center retrospective observational studies involved sufficient numbers of patients to demonstrate that the KDIGO classification is useful for the diagnosis of pediatric AKI. Moreover, as the KDIGO classification does not involve an estimation of the GFR but instead allows to stage AKI based on the $\mathrm{sCr}$, it can be considered superior to the pRIFLE classification. Therefore, we suggest the use of the KDIGO diagnostic criteria for pediatric AKI patients aged $\geq 3$ months. However, it must be noted that the use of the AKI diagnostic criteria has not yet been specifically evaluated in Japanese children.

Neonates and children aged $<3$ months possess a unique background that includes immaturity and perinatal factors; therefore, children under 3 months must be considered separately from those aged $\geq 3$ months. Although there have been investigations of the AKI diagnosis, treatment, and outcomes in neonates, there used to be no definitive diagnostic criteria for neonatal AKI [324, 325]. As the use of adult diagnostic tools such as the RIFLE, AKIN, and KDIGO criteria spread, their use for the diagnosis of AKI in neonates came to be researched too. Although the pRIFLE classification [318, 319] was proposed for pediatric use, it requires calculation of the eGFR and is therefore unsuitable for neonates, in whom the eGFR cannot be calculated. In 2014, Jetton et al. and Askenazi et al. introduced the neonatal 
modified KDIGO criteria (Table 14), which are based on the KDIGO diagnostic criteria [324, 325]. Similarly to the adult and pediatric KDIGO criteria, the neonatal modified KDIGO criteria define stages 1,2 , and 3 AKI primarily according to $\mathrm{sCr}$ values $1.5-1.9,2.0-2.9$, and $\geq 3$ times higher than baseline, respectively (Table 15). Although the baseline $\mathrm{sCr}$ is the minimum value prior to AKI diagnosis, it is only established at age $\geq 3$ months in Japan [317] and not in children under 3 months. The level of $\mathrm{sCr}$ in neonates immediately after birth is extremely close to the level of maternal $\mathrm{sCr}$ (generally $\leq$ $1 \mathrm{mg} / \mathrm{dL}$ ) [326]. It peaks at days $0-3$ and declines to a minimum value $(0.2-0.5 \mathrm{mg} / \mathrm{dL})$ over the following 1 week to 20 months [326-328]. (Note that prematurity (in terms of gestational age and birth weight) is reported to affect the postnatal sCr levels and the speed at which they decline [326, 328]). Going forward, it is necessary to collect data on Japanese neonates to establish their baseline $\mathrm{sCr}$ levels. It should be taken into consideration that the current absence of established baseline levels requires multiple measurements.

Koralkar et al. used the neonatal modified KDIGO criteria to examine AKI and mortality in 229 very low-birth-weight infants both at 36 weeks of gestational age and with a birth weight of 500-1500 kg [329]; the very low-birth-weight infants diagnosed with AKI had a significantly higher mortality than those not diagnosed with AKI. In an examination of 455 very low-birth-weight infants using the neonatal modified KDIGO criteria, Carmody et al. found AKI to be associated with mortality and prolonged hospitalization [330]. In addition, a gestational age $<28$ weeks was strongly associated with the onset of AKI; furthermore, all infants with a gestational age $<24$ weeks were diagnosed with AKI, which indicates an association between prematurity and AKI. Rhone et al. used the neonatal modified KDIGO criteria to examine the association between the AKI onset and nephrotoxic medications (acyclovir, amphotericin B, gentamicin, ibuprofen, indomethacin, iohexol, tobramycin, and vancomycin) in 107 very low-birth-weight infants; consequently, these drugs were shown to be associated with the onset of AKI [331]. In an examination of 96 neonates with moderate to severe asphyxia who underwent therapeutic hypothermia, Sarkar et al. demonstrated that abnormal brain MRIs at 7-10 days of age were significantly more frequent in infants diagnosed with AKI according to the neonatal modified KDIGO criteria [332]. As detailed above, many recent studies have employed the neonatal modified KDIGO criteria for the diagnosis of neonatal AKI.

\section{Literature review}

PubMed was searched for relevant studies published between January 1, 1980, and August 1, 2015, and papers related to the present CQ were identified from the search results.

\section{CQ9-2: Should biomarkers be used for the early diagnosis of AKI and for prediction of the survival outcomes in children?}

Recommendation: The use of biomarkers for the early diagnosis of AKI or to predict the survival outcomes cannot be recommended in children.

Strength of recommendation: Not graded

Quality of evidence: C

\section{Summary of evidence}

Many studies have indicated that biomarkers such as NGAL, cystatin C, L-FABP, IL-18, and KIM-1 may be useful for the early diagnosis of AKI and to predict the survival outcomes in children. However, interventions based on these indicators have not been reported to improve the renal or survival outcomes of AKI; therefore, their utility is limited.

\section{Commentary}

Neutrophil gelatinase-associated lipocalin (NGAL) is a secretory protein that has a molecular weight of $25,000 \mathrm{Da}$ and is secreted from activated neutrophils and tubular epithelial cells; the levels of NGAL in the blood and urine are known to be elevated in the hyperacute phase (i.e., the initial 2-4 h) of kidney injury. In an examination of 71 children undergoing a cardiopulmonary bypass (CPB) [333], the children who developed acute kidney injury (AKI) showed significantly elevated levels of serum and urinary NGAL 2 hours after the $\mathrm{CPB}$, with areas under the receiver operating characteristic curve (AUC) of 0.998 and 0.906, respectively; this

Table 15 Neonate modified KDIGO criteria

\begin{tabular}{lll}
\hline Stage & SCr criteria & $\cup O$ criteria \\
\hline Stage 0 & No change or rise in SCr of $<0.3 \mathrm{mg} / \mathrm{dL}$ & $\cup O \geq 1 \mathrm{~mL} / \mathrm{kg} / \mathrm{h}$ \\
Stage 1 & Increased in $\mathrm{sCr}$ of $\geq 0.3 \mathrm{mg} / \mathrm{dL}(48 \mathrm{~h}$ ) or increase to $1.5-1.9 \times$ baseline $(7$ days) & $\cup O<0.5 \mathrm{~mL} / \mathrm{kg} / \mathrm{h} \times 6 \mathrm{~h}$ \\
Stage 2 & Increase in sCr to $2.0-2.9 \times$ baseline & $\cup O<0.5 \mathrm{~mL} / \mathrm{kg} / \mathrm{h} \times 12 \mathrm{~h}$ \\
Stage 3 & Increase in $\mathrm{s} C \mathrm{r}>3.0 \times$ baseline or sCr $\geq 2.5 \mathrm{mg} / \mathrm{dL}$ or initiation of RRT & $\cup O<0.3 \mathrm{~mL} / \mathrm{kg} / \mathrm{h} \times 24 \mathrm{~h}$ or anuria $\times 12 \mathrm{~h}$ \\
\hline
\end{tabular}

Reference $\mathrm{sCr}$ will be defined as the lowest previous value. $\mathrm{s} C \mathrm{r}$ value of $2.5 \mathrm{mg} / \mathrm{dL}$ represents less than $10 \mathrm{~mL} / \mathrm{min} / 1.73 \mathrm{~m}^{2}$

$s C r$ serum creatinine, UO urine output, RRT renal replacement therapy 
study was the first to indicate the utility of biomarkers for the early diagnosis of AKI. An examination of 311 children undergoing cardiac surgery for congenital heart disease registered at three institutions [334] also indicated that the urinary NGAL is useful for the early diagnosis of AKI, despite a relatively low AUC of 0.71 . The urinary NGAL was also reported to be useful for the early diagnosis of AKI in a heterogeneous pediatric intensive care unit (PICU) patient cohort which had undergone mechanical ventilation and bladder catheterization [335]. Likewise, in a systematic review/meta-analysis of 19 studies [122], a subgroup analysis of 6 studies featuring populations of pediatric patients only demonstrated the utility of NGAL for the early diagnosis of AKI. With regard to the survival outcomes, two studies have reported that NGAL is significantly associated with mortality [336, 337].

Cystatin $\mathrm{C}$ is a low-weight molecular protein (molecular weight, approx. 13,000 Da) produced by nucleated cells all over the body. It is unaffected by environmental changes inside or outside the cells and is produced and secreted constantly; therefore, its concentration in the serum is constant. In addition, cystatin $\mathrm{C}$ is unaffected by factors such as inflammation, aging, the gender, the muscle mass, or exercise. The serum cystatin $C$ passes freely through the glomerular basement membrane and is filtered by the glomerulus. As more than $99 \%$ of the serum cystatin $C$ is reabsorbed by the proximal tubule and catabolized, healthy individuals excrete only a minimal amount of it in their urine. The serum cystatin $C$ has been indicated to be useful for early, accurate diagnoses of AKI. The cystatin $C$ concentrations in the serum and urine are known to increase 12-24 h after the onset of kidney injury. In an examination of 374 children undergoing CPB [338], AKI patients demonstrated significantly elevated serum cystatin C levels 12 and $24 \mathrm{~h}$ after the onset of AKI, with AUCs of 0.81 and 0.84 , respectively; thus, the serum cystatin $\mathrm{C}$ was shown to be a useful biomarker for the early diagnosis of AKI. It was also reported to be useful for the early diagnosis of AKI in a study of 288 children undergoing cardiac surgery [339]. While measurement of the serum cystatin $\mathrm{C}$ is covered by insurance in Japan, that of the urinary cystatin $\mathrm{C}$ is not.

Interleukin-18 (IL-18) is an inflammatory cytokine induced in the proximal tubule. In a study of 55 children undergoing $\mathrm{CPB}$ [340], children with AKI demonstrated a significant acute phase (4-6 h after $\mathrm{CPB}$ ) increase in their urinary IL-18 levels. The latter peaked at $12 \mathrm{~h}$ and remained high at $48 \mathrm{~h}$. The AUC at $12 \mathrm{~h}$ (i.e., at the urinary IL-18 levels' peak) was 0.75 , demonstrating the utility of the urinary IL-18 as a biomarker for the early diagnosis of AKI. In a systematic review/meta-analysis of 18 studies [341], the urinary IL-18 was also shown to be useful for the early diagnosis of AKI in a subgroup analysis of five studies featuring populations of pediatric patients only. Measurement of the urinary IL-18 is not covered by insurance in Japan.

The L-type fatty acid-binding protein (L-FABP), kidney injury molecule-1 (KIM-1), and albumin are also known to show a marked increase in urine as a result of kidney injury; these biomarkers have been studied for their potential utility in the early diagnosis of AKI. The L-FABP is a protein with a molecular weight of $14,000 \mathrm{Da}$ that is expressed in the liver, the small intestine, and the proximal tubular epithelial cells; since 2011, measurement of the L-FABP as a biomarker has been covered by insurance in Japan. In a study of 40 pediatric patients having undergone CPB [342], children who developed AKI demonstrated a significant acute phase $(4 \mathrm{~h}$ after AKI onset) increase in the urinary L-FABP. KIM-1 is a membrane-spanning glycoprotein expressed in the proximal tubular epithelial cells. In a study of 40 children undergoing $\mathrm{CPB}$ [343], children with AKI demonstrated a significant acute phase (12 h after CPB) increase in KIM-1. Moreover, in a prospective study of 294 children undergoing cardiac surgery, the urinary albumin/creatinine ratios $0-6 \mathrm{~h}$ after surgery were useful for the prediction of AKI [344].

Due to the diversity of the pathologies involved in AKI and the decline in the glomerular filtration rate (GFR), the use of a single biomarker to increase the accuracy of early diagnosis is of limited efficacy. One attempt to increase the accuracy of biomarkers for the diagnosis of AKI is to assemble a "panel" that combines multiple biomarkers and the renal angina index (RAI), an indicator of the risk of AKI onset $[345,346]$. Another advantage of panels is that, as each of the biomarkers that comprise them demonstrate favorable sensitivity and specificity at different periods, these different time phases may complement one another.

The uses of biomarkers have been studied in children, though not as often as in adults. Relevant studies indicate that these biomarkers may be useful for the early diagnosis of AKI and for the prediction of the survival outcomes. However, many of these studies involved relatively homogeneous populations, e.g., children undergoing CPB; the utility of these biomarkers has not been sufficiently assessed in populations of patients with diverse pathologies. Furthermore, the interventions based on these indicators have not yet been reported to improve the renal outcomes or survival outcomes of AKI; therefore, their utility is limited.

\section{Literature review}

PubMed was searched for relevant studies published between January 1980 and July 2015, and papers related to the present CQ were identified from the search results. 
CQ9-3: Should fluid overload be considered as a blood purification indication for pediatric AKI?

Recommendation: When determining whether blood purification is indicated in pediatric AKI, we suggest considering the fluid overload assessment in addition to absolute indications.

Strength of recommendation: 2

Quality of evidence: C

\section{Summary of evidence}

Many observational studies have reported that pediatric AKI non-survivors exhibit fluid overload compared to survivors. Few manuscripts have discussed fluid overload in neonatal AKI; there is little evidence to support prioritization of the fluid overload assessment for determination of the indication of blood purification in neonates.

\section{Commentary}

In pediatric acute kidney injury (AKI), life-threatening conditions resistant to conservative therapy, such as electrolyte disorders (hyperkalemia, etc.), fluid overload (pulmonary edema, heart failure, etc.) metabolic acidosis, and uremia symptoms (pericarditis, impaired consciousness, convulsions, etc.) are absolute indications for blood purification just like in adult AKI; in these cases, blood purification must be initiated immediately. However, for relative indications that are not considered to be immediately life-threatening, the criteria for the initiation of blood purification have not yet been defined. No randomized controlled trials have assessed the indications for blood purification and the timing of its initiation in pediatric AKI.

Although they were only observational studies, many recent papers have reported that fluid overload at the initiation of blood purification affects the survival outcomes. Body water is known to account for a larger percentage of the body weight in children than in adults. The percent fluid overload (\%FO) is considered to be useful for the assessment of fluid overload.

\section{\%Fluid overload (\%FO) \\ $=($ Fluid in-Fluid out $)$ \\ /PICU admission body weight \\ $\times 100(\%)$ Fluid in - Fluid out \\ : in-out balance before and after PICU admission}

In 2001, Goldstein et al. conducted a single-center study [347], followed by a large-scale multicenter study of continuous renal replacement therapy (CRRT) for pediatric AKI whose results were reported in 2005 [348]. This study examined the predictors of survival and death in 116 children registered in the Prospective Pediatric Continuous Renal Replacement Therapy (ppCRRT) Registry who underwent CRRT for multiple organ failure. Even when controlling for the severity of the illness (as measured by pediatric risk of mortality [PRISM] score), the \%FO at CRRT initiation was an independent predictor of survival; the \% $\mathrm{FO}$ was significantly lower in survivors than in non-survivors (survivors $14.2 \pm 15.9$ vs non-survivors: $25.4 \pm 32.9, p<0.05$ ), while the mortality was significantly higher when the $\% \mathrm{FO}$ was $>20 \%(<20 \%, 40 \%$ vs $>20 \%, 58 \%)$ at CRRT initiation. The same group later demonstrated that the $\% \mathrm{FO}$ at the initiation of blood purification was correlated with mortality (<10\%, 29.4\%; 10-20\%, 43.1\%; > 20\%, 65.6\%) [349]. Modem et al. reported that FO is a factor of poor survival outcomes [350]. Many studies of AKI in multiple organ failure [351353], stem cell transplantation [354], and extracorporeal membrane oxygenation (ECMO) following cardiac surgery $[355,356]$ have also reported that a lower \%FO at CRRT initiation is associated with more favorable survival outcomes. Similar results have also been reported in assessments of FO based on the body weight at hospital admission, at intensive care unit (ICU) admission, and at the initiation of blood purification [357]. Therefore, the early initiation of blood purification to prevent fluid overload may improve the survival outcomes; when determining whether blood purification is indicated in pediatric AKI, we suggest that the fluid overload assessment should be taken into consideration in addition to absolute indications.

However, all these results were obtained from observational studies; there is no high-quality evidence from interventional studies. In addition, a study of blood purification in children undergoing cardiac surgery failed to find an effective timing for initiation [358], while fluid overload was reported not to be an absolute predictor of the survival outcomes [359]. Unnecessary blood purification should be avoided in cases of mild AKI, in which the renal function recovers quickly. Blood purification carries serious complications, including catheter-related infection, an increased risk of bleeding from anticoagulation, and hemodynamic fluctuations unique to children of small constitution; therefore, the indication for blood purification and the timing of initiation must be considered comprehensively.

In neonatal AKI just like in pediatric AKI, renal replacement therapy (RRT) is considered when prolonged oliguria/anuria prevents the appropriate adjustment of the body fluid, electrolytes, and blood nitrogen level. The overall mortality in neonatal AKI is reported to range between 11.3 and $48.3 \%$ [360-370], while the mortality is reported to be $4.1-71.7 \%$ in premature neonates [329, 371-374], 13.9-70.0\% in asphyxiated neonates [367, 375, 376], 2.9-11.6\% in neonates undergoing a cardiopulmonary bypass/cardiac surgery [377-379], 71.2\% in sepsis [380], and $50-100 \%$ in neonates with AKI who undergo blood purification [361, 365-367]. Due to the different definitions of AKI in these studies and to the 
major differences in the standards of neonatal intensive care medicine between countries and institutions, uniform comparisons of past studies are difficult. The risk factors for death in neonatal AKI include mechanical ventilation, hypervolemia ( $\% \mathrm{FO} \geq 7 \%$ ), chronic heart failure, a low birth weight, hypoxia, oliguria/anuria, dialysis, and metabolic acidosis [360]. The risk of death is particularly high in neonates with oliguria [360-364, 369, 375, 376]. However, no studies have discussed FO in neonatal AKI; there is little evidence to support prioritization of the fluid overload assessment when determining the indication of blood purification in neonates. Low-birth-weight infants present technical problems such as vascular access; however, the indication for acute blood purification in neonates must be determined comprehensively on a case-by-case basis.

\section{Literature review}

PubMed was searched for relevant studies published between January 1980 and July 2015, and papers related to the present CQ were identified from the search results. Manuscripts that supplemented the commentary were hand searched as appropriate.

\section{CQ9-4: What modalities of blood purification therapy should be selected for pediatric AKI patients?}

Recommendation: For pediatric AKI patients requiring blood purification, an appropriate modality tailored to the patient's constitution and disease condition should be considered.

Strength of recommendation: Not graded

Quality of evidence: D

\section{Summary of evidence}

Observational studies of children and neonates who underwent CRRT or other modalities of blood purification have been performed. However, there has been no evidence to demonstrate the effects of different blood purification modalities on the outcomes nor of their superiority to peritoneal dialysis.

\section{Commentary}

The modalities of blood purification for acute kidney injury (AKI) include peritoneal dialysis (PD), extracorporeal intermittent renal replacement therapy (IRRT), and continuous renal replacement therapy (CRRT). In the past, PD was often the first choice; however, due to progress in the techniques of vascular access, in the types of catheters, the hemodialysis (HD) devices, and the pediatric intensive care management, extracorporeal CRRT has become more common. At present, the only studies that have compared PD and extracorporeal CRRT are observational studies [381, 382], and there is no evidence that one modality is superior to the other. However, as in adults, CRRT is considered preferable to IRRT for hemodynamically unstable patients. Many evidences that inform the blood purification modality selection for adults can be applied pediatric AKI; however, the incidence of pediatric AKI is $<1 \%$ in all hospitalized children [383] and $4.5 \%$ in children admitted to the ICU [384]. Differences in knowledge and health care resources between regions and institutions may greatly affect the selection of blood purification modalities. Further investigations are necessary to better inform the selection of suitable blood purification modalities.

In children (aside from neonates), blood purification can be performed safely with a combination of low priming volume and multipurpose blood purification devices. For vascular access, the size of the catheter is chosen to suit the patient's constitution (Table 16). The standard values for the quantity of blood flow (QB), the dialysate flow rate (QD), and the filtration rate $(\mathrm{QF})$ are $1-5 \mathrm{~mL} / \mathrm{kg} / \mathrm{min}, \mathrm{QB} \times 0.2-2.0$, and $0-20 \%$ of the $\mathrm{QB}$, respectively. With regard to circuit priming before the initiation of HD, a priming volume of $\geq 10 \%$ of the circulating blood volume causes hypotension at the initiation

Table 16 Blood purification for pediatric patients

\begin{tabular}{|c|c|c|c|c|c|c|c|c|c|c|c|c|c|}
\hline \multicolumn{2}{|l|}{ Bodyweight (kg) } & $0 \sim$ & 1 & 2 & 3 & & 5 & & 10 & & 15 & 20 & 25 \\
\hline \multicolumn{2}{|c|}{ Filter surface area $\left(\mathrm{m}^{2}\right)$} & & & 0.03 & & & & & 0.3 & & 0.7 & & \\
\hline \multirow[t]{3}{*}{ Priming } & Packed red blood cells \pm albumin (A) & A & A & A & A & A & A & $\mathrm{A} / \mathrm{B}$ & C & C & C & C & C \\
\hline & Albumin (B) & & & & & & & & & & & & \\
\hline & Saline $(C)$ & & & & & & & & & & & & \\
\hline \multicolumn{2}{|l|}{ Catheter size } & $18 \mathrm{G}$ & $17 G$ & & $6 \mathrm{Fr}$ & & $7 \mathrm{Fr}$ & & $8 \mathrm{Fr}$ & & $9 \mathrm{Fr}$ & $10 \mathrm{Fr}$ & $11 \mathrm{Fr}$ \\
\hline QB & $1 \sim 5(\mathrm{~mL} / \mathrm{min}) \times$ bodyweight & & & & $3 \sim 15$ & & & & $10 \sim 50$ & & & & \\
\hline QD & $\mathrm{QB} \times(0.2 \sim 2.0)$ & & & & $36 \sim 1800$ & & & & $120 \sim 6000$ & & & & \\
\hline QF & $\mathrm{QB} \times(0 \sim 0.2)$ & & & & $0 \sim 180$ & & & & $0 \sim 600$ & & & & \\
\hline \multirow[t]{2}{*}{ Anticoagulation } & Nafamostat mesilate & & & & $1.5 \sim 3 \mathrm{mg} / \mathrm{h}$ & & & & $5 \sim 10 \mathrm{mg} / \mathrm{h}$ & & & & \\
\hline & Heparin & & & & 30 60 unit/h & & & & 100 200 unit/h & & & & \\
\hline
\end{tabular}

$Q B$ quantity of blood flow, $Q D$ dialysate flow rate, $Q F$ filtration rate 
of dialysis; therefore, priming with blood products is preferred [385]. After priming with blood products, it is recommended dialyzing the priming blood in the circuit to adjust the electrolyte and acid-base balance and by removing the potassium and citric acid in the blood products.

In the past, PD used to be the method of choice for low-birth-weight infants (including neonates) due to the technical problems. However, extracorporeal blood purification can recently be performed safely. Extracorporeal blood purification has been technically possible in Japan since 2001, when blood purification devices (QB can be adjusted from $1 \mathrm{~mL} / \mathrm{min}$ ), filters, and other related devices became commercially available. In 2013, the Pediatric Acute Blood Purification Handbook described blood purification in neonates and the Guideline for Neonatal Extracorporeal Blood Purification was published in Japan. In addition, the Neonatal Extracorporeal Blood Purification Manual was published in 2014. Meanwhile, there have been many reports on blood purification primarily in pediatric patients (including some neonates) both in Japan and outside of Japan [348, 386, 387].

In blood purification for low birth-weight-infants (including neonates), vascular access is a specific and important issue; in addition to the standard central venous route, the umbilical arteries/veins and peripheral arteries can also be used (when the flow rate is low, the peripheral veins can sometimes also be used). There has been a Japanese case report of blood purification in an infant weighing < $500 \mathrm{~g}$; however, blood purification in infants weighing $<2 \mathrm{~kg}$ is considered to require experienced skill. Central venous catheter size should be large. Although variations between institutions exist, the catheter sizes used for infants weighing 1,2 , and $3 \mathrm{~kg}$ are generally $17 \mathrm{G}, 15 \mathrm{G}$, and $6 \mathrm{Fr}$, respectively. As in pediatric patients, the circuit is basically primed with mixed blood in order to prevent hypotension. The blood preparation is recycled and dialyzed to remove potassium and citric acid before initiating the blood purification. Prevention for hypothermia is necessary. Details are described in the above-cited guidelines and handbooks (Table 16).

Outside of Japan, the blood purification of low-birthweight infants (including neonates) was primarily consisted of peritoneal dialysis [388-390]. The improvements in blood purification devices have recently led to an increase in extracorporeal acute blood purification [391-393]. However, there have been no randomized controlled trials (RCTs) to examine the performance of extracorporeal blood purification in Japan or elsewhere.

The optimal blood purification modality also depends on the disease conditions. For AKI with acute brain injury, intracranial hypertension, or cerebral edema, CRRT (continuous hemodiafiltration [CHDF] or $24 \mathrm{~h}$ of $\mathrm{PD}$ ) is recommended, as IRRT may cause intracranial hypertension, dialysis disequilibrium syndrome, and reduced blood pressure [394].

\section{Literature review}

PubMed was searched for relevant studies published between January 1980 and July 2015, and papers related to the present CQ were identified from the search results. The literature needed for the commentary was manually extracted from PubMed as appropriate.

\section{CQ9-5: How should therapeutic strategies be discussed and determined in cases of neonates and children with AKI who have serious impairments and poor survival prognoses?}

Recommendation: Medical care staff should first consider the patient's present status and survival prognosis discuss the indication for renal replacement therapy among themselves. Afterwards, they should explain the advantages and disadvantages of the different treatments to the patient's family and consult with them about suitable therapeutic strategies. Each patient should be dealt with as appropriate on a case-by-case basis and with reference to the "Guideline on Determining Medical Care of Children with Serious Diseases" of the Japan Pediatric Society.

Strength of recommendation: Not graded

Quality of evidence: D

\section{Summary of evidence}

Despite the existence of multiple case reports and case series, there is no relevant high-level evidence.

\section{Commentary}

Children with severe motor and intellectual disabilities caused by factors such as chromosomal abnormalities, multiple abnormality syndromes, and neonatal asphyxia (cerebral hypoxia) are estimated to occur in approximately 0.3 out of 1000 live births. Factors such as severe asphyxia and infection frequently cause acute kidney injury (AKI) in neonates. Neonates with AKI necessitating blood purification often present comorbid serious brain injury. Children with severe motor and intellectual disabilities that correspond to grades 1-4 of Oshima's classification have a high risk of developing severe infections and frequently require renal replacement therapy (RRT) for AKI. However, most past reports of RRT in children with severe impairments, mostly from Japan, have involved the issues of chronic dialysis. Out of a total of 23 reports (37 patients), 20 of them (32 patients) described the initiation (or scheduled initiation) of peritoneal dialysis (PD), 2 (4 patients) described the initiation of chronic hemodialysis (HD), and 1 (1 patient) described PD and continuous hemodiafiltration (CHDF) for AKI. In another report, the patient was treated without initiating 
dialysis. Many of these reports have suggested that a multidisciplinary health care team should consider the patient's case and should ultimately decide what to do after consulting the patient's family. Meanwhile, reports from outside of Japan have stated that the frequency of peritonitis in PD for children with psychomotor retardation is the same as in children without psychomotor retardation if dedicated cooperation and support are provided. There have also been reports of dialysis initiation in children with chromosomal abnormalities [395-397]. These reports have demonstrated that RRT can be performed relatively safely in children with severe motor and intellectual disabilities. However, the medical staff experience a great physical and psychological burden; therefore, the health care team also needs support.

There are no definitive criteria upon which to determine the indication for RRT in children with severe impairments; thus, it must be considered on a case-by-case basis. The health care team should decide on a therapeutic strategy after considering the patient's present status and long-term survival prognosis among themselves, explaining the nature of the treatments to the patient's family, and presenting the respective advantages and disadvantages of treatment versus no treatment. Various guidelines [30-32] can be referred as well. This concept is called shared decision-making; essentially, health care professionals must share information with the patient's family and decide on a therapeutic strategy together. The process is described below.

\section{Therapeutic strategy discussion among the health care team}

Before providing information to the patient's family, the health care staff must gather information and share it among themselves in order to determine the patient's present status. Discussions should not only include the attending physician's department, but also intensive care specialists, neonatal intensive care specialists, and nurses; when necessary, clinical psychologists, a palliative care team, medical social workers, and other departments and disciplines should also be included. Based on these discussions, conceivable treatments should be identified as options, and the problems and invasiveness of each option should be abstracted (for example, for acute blood purification, these include complications associated with catheter insertion, the risk of hypotension associated with dialysis initiation, blood transfusion). Suitable strategies are then examined based on a prediction of the patient's prognosis (survival prognosis and sequelae) and on the consideration of the advantages and disadvantages of the potential treatments.

When considering withholding or discontinuing treatment, the relevant facility's institutional review board may be convened, or a conference may be held to discuss ethical issues.

\section{Explanation to the patient's family}

When explaining therapeutic strategies to the patient's family, the parents must always be present; other individuals may attend the explanation if requested by the parents (grandparents, etc.). The name of the child's illness, its disease condition, the respective advantages and disadvantages of treatments such as blood purification (including their complications) versus no treatment, and the prognosis (sequelae and survival prognosis) should be explained comprehensively in a way that is easy to understand. Important information should be provided in writing. Moreover, even when acute blood purification is to be performed, it must be explained that permanent RRT may be necessary, thereby placing a burden on the patient and their family (which also requires explanation). In addition, the family must be informed that even after a strategy is decided, it can be reconsidered if they change their minds.

The content of this explanation, the way it is explained, and the course by which a strategy is chosen must be written in the patient's medical record. In particular, when treatment is withheld, it is important to record the course and content of the discussion that led to the treatment withdrawal. When the patient's family and the health care team cannot agree on a strategy, advice should be sought from a committee comprising the institutional review board and many other experts.

\section{Subsequent follow-up and reconsideration of the treatment strategy}

Even after a strategy is decided, the patient's family will require continuous mental support. After blood purification is initiated, the patient's impairment may progress irreversibly, thereby requiring discontinuation of the treatment. On the other hand, even if the patient's family initially decides not to perform the treatment, the treatment may later be performed if they change their minds (or for other reasons). These reconsiderations of the therapeutic strategies require a new round of discussion. Moreover, when changing the therapeutic strategy, a consensus must be obtained among the health care team as appropriate.

If the patient's family wishes to discontinue dialysis, it is necessary to confirm that this is not based on temporary emotion but on a careful consideration and sufficient understanding of the child's status. The patient may also die shortly after discontinuing treatment; therefore, when deciding to do so, the timing of the discontinuation must also be discussed.

The above procedure is also followed for comorbid severe brain injury associated with acquired causes, such 
as acute encephalitis/encephalopathy and head trauma. When the patient him/herself is evidently conscious and is capable of expressing their will but has a poor survival prognosis (such as in the case of older children with terminal malignancy), the patient's own will must be respected and prioritized above all else. In such cases, the question of how much medical information to convey to the patient must first be discussed with the patient's family and agreed in advance.

\section{Literature review}

PubMed and Ichushi-Web (Japanese language) were searched for relevant studies published up to August 30, 2015, and papers related to the present CQ were identified from the search results. References in Japanese are not shown in this article.

\section{Chapter 10: AKI in the elderly and ethical aspects Aging as a risk factor for AKI occurrence}

As Japan has become a super-aged society, it is increasingly crucial to understand the pathologies in which age is a risk factor and to take preemptive measures to prevent these pathologies in order to tackle diseases with no established treatments. A typical example of these pathologies is acute kidney injury (AKI). Elderly people account for a large and constantly increasing percentage of AKI patients [398]. In addition, many observational studies published in the last 25 years have found aging to be a significant risk factor for AKI onset [34, 399].

Pre-AKI renal impairment is a risk factor for AKI; chronic kidney disease (CKD) patients are at a high risk of developing AKI. With the base of hypertensive nephrosclerosis, aging is associated with a reduced glomerular filtration rate (GFR); therefore, aging is conceivably an underlying factor of CKD, which in turn can be considered a universal risk factor for AKI [400, 401]. In the present guideline's examination of the risk factors for individual AKI, age was considered to be an independent risk factor for the onset of AKI in cardiac surgery ("CQ3-1: What should be assessed as risk factors for AKI development in cardiac surgery?" section), acute heart failure ("CQ3-3: What should be assessed as risk factors for AKI development in heart failure?" section), and sepsis ("CQ3-4: What should be assessed as risk factors for AKI development in sepsis?" section). Although it is not covered by any CQ in the present guideline, dehydration-induced pre-renal AKI, which is an important aspect of community-acquired AKI, restricts the renal blood flow in elderly people due to their already low fluid volume and to arteriosclerosis; therefore, elderly people are at a high risk of developing dehydration-induced AKI [402]. Drugs such as renin-angiotensin-aldosterone system inhibitors, diuretics, non-steroidal anti-inflammatory drugs (NSAIDs), and vitamin D preparations (which cause hypercalcemia) - the latter two of which are often used by elderly people-are involved in the increase in AKI among the elderly [403]. Elderly people are also known to be at a high risk of drug-induced AKI (contrast agents, aminoglycosides, etc.) [404, 405]. Therefore, in order to prevent AKI, elderly people's exposure to these drugs must be minimized. Urinary tract obstructive kidney injury and ANCA-associated, vasculitis-induced rapidly progressive glomerulonephritis (RPGN) are also common causes of AKI in the elderly.

The present guideline recommends the use of the KDIGO criteria for the diagnosis of AKI; however, caution is necessary in applying these criteria to elderly patients. Although the KDIGO diagnostic criteria for AKI depend on the baseline renal function, it is often unknown in clinical settings; therefore, it is permissible to use the serum creatinine (sCr) as back-calculated from the MDRD formula by assuming an estimated glomerular filtration rate (eGFR) of 75 (or from the sCr-eGFR predictive equation for Japanese people). However, a standard eGFR of 75 often overestimates the renal function in elderly people; consequently, the $\mathrm{sCr}$ as back-calculated from eGFR formulas is underestimated, which causes an increase in false positives in the AKI diagnosis (overdiagnosis). Due to their diminished capacity for renal recovery, AKI easily progresses to a severe state in elderly people. Because of the physical frailty and of cardiovascular complications, it is highly likely that the survival and renal outcomes predicted for adults do not apply to elderly people. Based on the above, elderly people, who are at a high risk of developing AKI, require highly accurate AKI diagnoses; therefore, the development of dedicated AKI diagnostic criteria for elderly people may be needed.

\section{Blood purification in elderly AKI patients}

Aging is an evident high-risk factor for AKI development [34, 399]. In Japan and other developed nations, the incidence of AKI has been increasing as the population ages; this trend is particularly pronounced in men [398, 406, 407]. Blood purification is more often required in elderly patients (particularly those aged over 75) [408]. In a Turkish observational study, blood purification was performed in 43 of 193 patients (22\%) with a mean age of 79.99 years who were diagnosed with AKI as defined by the KDIGO classification; when including the 16 patients $(12.7 \%)$ who required blood purification after discharge, a total of $37.7 \%$ of the patients required blood purification [409].

In AKI patients-including elderly ones-whose AKI progresses to an advanced stage and presents uremic symptoms, blood purification undoubtedly improves the survival outcomes [410]. However, there have been no randomized controlled trials (RCTs) that enrolled elderly patients with advanced AKI with the survival outcome 
as the primary endpoint nor have there been any relevant systematic reviews. However, there has been a retrospective cohort study of elderly AKI patients. Liu et al. examined the factors that affected the survival outcomes of 41 elderly AKI patients aged $80-100$ years who required continuous renal replacement therapy (CRRT) in Beijing, China [411]. In the AKI patients who underwent CRRT, the APACHE II score was the factor most strongly associated with the survival outcomes; the number of involved organs and hypoalbuminemia was also indicated to be important, while the age itself was unrelated to the survival outcomes. These results are not limited to elderly patients, but are relatively common to all AKI patients; they may indicate that if AKI has reached an advanced stage, blood purification should be considered even in elderly patients. However, in a similar study by Kayatas et al. involving patients within a slightly broader age range ( $\geq 65$ years), a reduced blood pressure, high CRP levels, and low hemoglobin $(\mathrm{Hb})$, aging was also found to be associated with the AKI outcomes [409]. Moreover, several studies have noted racial differences in the outcomes; among elderly ICU patients who required blood purification, the outcomes were worse for non-Caucasian patients [412, 413].

In elderly AKI patients, the outcomes are often affected not only by AKI but also by existing comorbidities. This is also observed in maintenance hemodialysis (HD) patients and elderly people in general [414]. Elderly AKI patients in ICUs are reported to commonly exhibit evident dementia and symptoms of delirium [412]. In addition, the AKI morbidity is high in frail elderly people; the latter is highly likely to require blood purification, and their activities of daily living are reported to decline progressively [ $[413,415]]$. Therefore, when considering whether to perform blood purification in an elderly AKI patient, the chronological age alone is not sufficient; the severity of the AKI, the speed of its progression, and details of the patient's pre-AKI health status may also be necessary. The above has also been stated in a limited number of studies. However, in elderly AKI patients who did not demonstrate any major health problems before developing AKI, we do not recommend the needless avoidance of blood purification simply because of age. Conversely, AKI patients with multiple comorbidities and low activities of daily living before developing AKI are highly likely to have a poor renal and survival prognosis, which makes it necessary to consider whether blood purification should be performed or not [415]. Giving a definitive answer to this question would require prospective RCTs involving large numbers of elderly AKI patients, as well as sub-analyses to determine the effective groups. Coca at Yale University recommends that such RCTs involving elderly AKI patients should be conducted [416].
On the patient's side, medical economic factors would normally be the greatest determinants of whether to undergo blood purification; however, since Japan has abundant public health insurance, medical economic aspects (burdens of expenses) do not have a major impact on patients' decisions. Therefore, the decision whether to undergo blood purification is considered with social factors on the patient's side, medical perspectives, and the medical institution's treatment capacity on the medical side.

\section{Progression from AKI to CKD in elderly patients}

The renal outcomes of AKI are not favorable. Observational studies have shown that $20-50 \%$ of AKI survivors progress to CKD. AKI is not only involved in the de novo development of CKD, but it may also accelerate existing CKD. When AKI develops in a person with a previously normal renal function, if the renal function does not recover to pre-AKI status, one of the following three pathways will unfold: (1) progression to end-stage kidney disease (ESKD) after the onset of AKI (AKI to ESKD), (2) incomplete recovery of the renal function from AKI and progression to CKD (AKI to CKD), and (3) temporary recovery of the renal function from AKI, but subsequent progression to CKD (AKI to subclinical CKD). Furthermore, it has been shown that $30 \%$ of AKI patients have underlying CKD. This represents a fourth pathway: AKI to worsening CKD.

The prevalence of CKD in adults is estimated to be $\geq$ $10 \%$. As the renal function declines with age, the prevalence of CKD is higher in the elderly; CKD affects 30-40\% of people aged $\geq 65$ years. Aging has been identified as a risk factor for post-AKI progression to CKD, along with diabetes, hypertension, heart failure, renal impairment, and hypoalbuminemia. The differences in the AKI incidence and the renal function outcomes of elderly and non-elderly people have not been examined in sufficient detail. However, in light of the high prevalence of CKD in the elderly and of the involvement of aging itself in the risk of progression from AKI to CKD, it is rather unlikely that the post-AKI renal function outcomes would be more favorable in elderly people than in non-elderly people. It is necessary to pay attention to the prevention and early detection of AKI, and its progression to a severe state.

The renal outcomes of AKI in elderly people were analyzed in a study of people enrolled in Medicare, the American health insurance system for the elderly (aged $\geq 65$ years) [417]. Of the more than 230,000 people examined, CKD was present in $12 \%$, while AKI had developed in $3.1 \%$. Of the people who had developed AKI, $34 \%$ had prior CKD (AKI + CKD). The post-AKI survival rates of $A K I+C K D$ patients were worse than those of patients with AKI alone. This study also analyzed the risks of progression to ESKD within 2 years of discharge; the hazard ratios for the development of ESKD in AKI + 
CKD, AKI only, and CKD only were 41.19, 13.0, and 8.43, respectively. These results indicated that elderly people with CKD who develop subsequent AKI experience poor renal outcomes.

The severity and frequency of AKI have also been reported to be independently involved in the risk of progression to CKD. One study retrospectively analyzed the relationship between the post-hospitalization development of AKI and the prognosis in a cohort of elderly people hospitalized for myocardial infarction who were Medicare beneficiaries [418]. In the analysis-in which the patients were divided into four quartiles based on $\mathrm{sCr}$ increases of $0.1-3.0 \mathrm{mg} / \mathrm{dL}-$ the quartile of patients with the largest percentage increase in $\mathrm{sCr}$ demonstrated high rates of pre-existing diabetes, hypertension, myocardial infarction, congestive heart failure, and cerebrovascular injury, as well as a reduced renal function. After adjusting for these factors, the percentage increase in the $\mathrm{sCr}$ demonstrated significant correlations with the percentage of post-AKI progression to end-stage renal failure and death. In elderly patients, the AKI severity was associated with the renal and survival outcomes. An association was also observed between the number of AKI episodes and the rate of progression to CKD. In a study of American veterans with comorbid diabetes, patients with multiple AKI episodes demonstrated a higher rate of progression to stage G4 CKD than those with a single AKI episode [419]. Given that a high percentage of elderly people have CKD and that the post-AKI renal function outcomes are poor in patients with comorbid CKD, practitioners should be cautious about the development of $\mathrm{AKI}$ in non-elderly and older people.

\section{Ethical considerations relevant to AKI treatment in elderly people}

Elderly people are at a high risk of AKI and have poorer renal and survival outcomes than young people. This point has already been discussed in the present guideline, and it has also been covered in many studies [415, 417, 420]. In a study comparing dialyzed AKI patients with not-yet-dialyzed AKI patients, Wilson et al. concluded that dialysis causes more harm than no dialysis when the $\mathrm{sCr}$ is below $3.8 \mathrm{mg} / \mathrm{dL}$; the authors' opinion was that dialysis is harmful in the presence of a decreased muscle mass, i.e., in frail patients [413]. The message of these findings is that in elderly patients, AKI must be recognized not as a transient disease condition that can be cured but rather as a serious status that leads to prolonged hospitalization, more complications, and a higher risk of death. Moreover, the decision to initiate dialysis in elderly AKI patients must be understood not only in terms of its effects on the prognosis (i.e., the survival prognosis, progression to chronic dialysis) but also of its major effects on the patient's quality of life (QOL).
Points of note in relation to elderly AKI patients' treatment Crews et al. demonstrated that in elderly AKI patients, earlier dialysis initiation may in fact cause harm. The implementation of shared decision-making (as described below) for dialysis initiation enables more patient-centered care, and the elderly who participate in this process tend to forgo dialysis initiation [421]. When deciding whether to initiate dialysis in an elderly AKI patient, it is important to make a comprehensive determination of the indication based on more than the disease condition and to obtain consent from the patient (or their guardian) by dialoguing with them. This process, which is called "shared decision-making," is described in a guideline published by the Renal Physicians Association in the United States entitled "Shared Decision Making in the Appropriate Initiation of and Withdrawal from Dialysis, 2nd Edition." We present here a proposed "dialysis assessment form for elderly AKI patients," which is based on a modification of the above guideline. Another potentially helpful resource is a process notebook that was developed in Japan to consider the initiation of dialysis in elderly patients with the patient's cooperation. Going forward, we hope that this perspective will lend further momentum to shared decision-making in the consideration of elderly AKI patients' treatment.

The aging of society and the consequent increase in social welfare expenses

The Japanese society has been aging at a rate that is unparalleled in the world, and this trend is predicted to continue. In the 2014 fiscal year (FY), the aging rate in Japan reached 26.0\%; specifically, individuals aged $65-74$ years accounted for $13.4 \%$ of the overall population, while those aged $\geq 75$ years represented $12.5 \%$ of the population. The aging rate is predicted to exceed $30 \%$ by 2025 . In FY 2012, Japan's total social welfare expenditure reached $\backslash 108.5568$ trillion ( $\$ 977$ billion), its highest level ever. The percentage of national income spent on social welfare expenses has risen from $5.8 \%$ in 1970 to $30.9 \%$, and the benefits paid to the elderly accounted for $68.3 \%$ of these expenses in FY 2012. Of the approximately $\backslash 45$ trillion $(\sim \$ 405$ billion) spent on national health care in FY 2012, approximately $\backslash 18$ trillion $(\sim \$ 162$ billion; $44 \%)$ was spent on late-stage elderly people (age $\geq 75$ years, $12.5 \%$ of the overall population). It must be recognized that these medical economic factors may also have a considerable influence on the treatment of AKI in elderly patients.

\footnotetext{
Acknowledgements

The Japanese Clinical Practice Guideline for Acute Kidney Injury 2016 Committee.

Kiyoshi Mori, School of Pharmaceutical Sciences, University of Shizuoka,

Shizuoka, Japan.

Yoshihiro Taniyama, Department of Nephrology, Kindai University Faculty of

Medicine, Osaka, Japan.

Shu Wakino, Department of Internal Medicine, School of Medicine, Keio

University, Tokyo, Japan.

Hideo Yasuda, Internal Medicine 1, Hamamatsu University School of

Medicine, Shizuoka, Japan.
} 
Shinji Kume, Department of Medicine, Shiga University of Medical Science, Shiga, Japan.

Tadashi Sofue, Division of Nephrology and Dialysis, Department of Cardiorenal and Cerebrovascular Medicine, Kagawa University, Kagawa, Japan. Kiichiro Fujisaki, Department of Medicine and Clinical Science, Graduate School of Medical Sciences, Kyushu University, Fukuoka, Japan. Hideaki Shima, Division of Nephrology, Department of Internal Medicine, Osaka Medical College, Osaka, Japan.

Koji Tomori, Department of Nephrology, Faculty of Medicine, Saitama Medical University, Saitama, Japan.

Taro Horino, Department of Endocrinology, Metabolism and Nephrology, Kochi Medical School, Kochi, Japan.

Yusuke Watanabe, Division of Dialysis Center and Department of Nephrology, Saitama Medical University International Medical Center, Saitama, Japan.

Hiroki Hayashi, Department of Nephrology, Fujita Health University School of Medicine, Aichi, Japan.

Takeshi Moriguchi, Department of Emergency and Critical Care Medicine, University of Yamanashi School of Medicine, Yamanashi, Japan. Kazuto Yamashita, Department of Healthcare Economics and Quality Management, Graduate School of Medicine, Kyoto University, Kyoto, Japan. Ryota Inokuchi, Department of Acute Medicine, The University of Tokyo, Tokyo, Japan.

Kensuke Nakamura, Department of Emergency and Critical Care Medicine, Hitachi General Hospital, Ibaraki, Japan.

Yoshitaka Hara, Department of Anesthesiology, Critical Care Medicine, Fujita Health University School of Medicine, Aichi, Japan.

Kengo Furuichi, Department of Nephrology, Kanazawa University Hospital, Ishikawa, Japan.

Sho Sasaki, Center for Innovative Research for Communities and Clinical Excellence, Fukushima Medical University, Fukushima, Japan.

Takayuki Tsuji, Internal Medicine 1, Hamamatsu University School of Medicine, Shizuoka, Japan.

Hiroyuki Yamada, Department of Nephrology, Graduate School of Medicine, Kyoto University, Kyoto, Japan.

Sayoko Yonemoto, Department of Internal Medicine, Hyogo Prefectural Nishinomiya Hospital, Hyogo, Japan.

Taka-aki Nakada, Department of Emergency and Critical Care Medicine, Chiba University Graduate School of Medicine, Chiba, Japan.

Noriyuki Hattori, Department of Emergency and Critical Care Medicine, Chiba University Graduate School of Medicine, Chiba, Japan.

Tetsushi Yamashita, Department of Nephrology and Endocrinology, The University of Tokyo, Tokyo, Japan.

Zentaro Kiuchi, Department of Pediatrics, Kyorin University School of

Medicine, Tokyo, Japan.

Mariko Sawada, Department of Pediatrics, Kurashiki Central Hospital,

Okayama, Japan.

Masaki Takahashi, Department of Pediatrics, Tokyo Medical and Dental University, Tokyo, Japan.

Masanori Tani, Department of Critical Care, Saitama Children's Medical Center, Saitama, Japan.

Yusuke Nakazawa, Department of Neonatalogy, Shizuoka Children's Hospital, Shizuoka, Japan.

Masaki Nunoyama, Department of Pediatrics, Showa University Fujigaoka Hospital, Kanagawa, Japan.

Tsuguya Fukui, St. Luke's International Hospital, Tokyo, Japan.

Seiichi Matsuo, Department of Nephrology, Nagoya University Graduate

School of Medicine, Aichi, Japan.

Shoichi Maruyama, Department of Nephrology, Nagoya University Graduate School of Medicine, Aichi, Japan.

Motoko Yanagita, Department of Nephrology, Kyoto University Graduate School of Medicine, Kyoto, Japan.

Kazuhiko Tsuruya, Department of Integrated Therapy for Chronic Kidney Disease, Graduate School of Medical Sciences, Kyushu University, Fukuoka, Japan.

\section{Authors' contributions}

All authors read and approved the final manuscript.

\section{Competing interests}

The authors declare that they have no competing interests.

\section{Publisher's Note}

Springer Nature remains neutral with regard to jurisdictional claims in published maps and institutional affiliations.

\section{Author details}

${ }^{1}$ Department of Acute Medicine, The University of Tokyo, Tokyo, Japan. ${ }^{2}$ Department of Anesthesiology and Critical Care Medicine, Fujita Health University School of Medicine, Toyoake, Aichi, Japan. ${ }^{3}$ Department of Nephrology, Wakayama Medical University, Wakayama, Japan. ${ }^{4}$ Department of Emergency and Critical Care Medicine, Tokyo Women's Medical University Yachiyo Medical Center, Chiba, Japan. ${ }^{5}$ Kidney Center, Department of Surgery, Nikko Memorial Hospital, Hokkaido, Japan. ${ }^{6}$ Clinical Research Support Center, Tomishiro Central Hospital, Okinawa, Japan. ${ }^{7}$ Department of Nephrology, Fujita Health University School of Medicine, Toyoake, Aichi, Japan. ${ }^{8}$ Department of Nephrology and General Internal Medicine, Saitama Medical University, Saitama, Japan. ${ }^{9}$ Division of Anticipatory Molecular Food Science and Technology, Department of Diabetology and Endocrinology, Kanazawa Medical University, Kanawaza, Ishikawa, Japan. ${ }^{10}$ Department of Community Medical Supports, Tohoku Medical Megabank Organization, Tohoku University, Sendai, Japan. ${ }^{11}$ Division of Nephrology and Hypertension, St. Marianna University School of Medicine, Kawasaki, Kanagawa, Japan. ${ }^{12}$ Department of Emergency and Critical Care Medicine, University of Yamanashi School of Medicine, Yamanashi, Japan. ${ }^{13}$ Blood Purification Unit, Hamamatsu University Hospital, Hamamatsu, Japan. ${ }^{14}$ Department of Kidney Disease and Hypertension, Osaka General Medical Center, Osaka, Japan. ${ }^{15}$ Nephrology and Blood Purification, Saitama Medical Center, Saitama Medical University, Saitama, Japan. ${ }^{16}$ Department of Nephrology, Graduate School of Medicine, Kyoto University, Kyoto, Japan.

${ }^{17}$ Department of Nephrology and Endocrinology, The University of Tokyo, Tokyo, Japan. ${ }^{18}$ Division of Nephrology and Rheumatology, National Center for Child Health and Development, Tokyo, Japan. ${ }^{19}$ Department of Nephrology, Shizuoka Children's Hospital, Shizuoka, Japan. ${ }^{20}$ Department of Nephrology and Hypertension, Kawasaki Medical School, Okayama, Japan. ${ }^{21}$ Health Care Division, Health and Counseling Center, Osaka University, Osaka, Japan. ${ }^{22}$ Department of Endocrinology, Metabolism and Nephrology, Kochi Medical School, Kochi University, Kohasu, Oko-cho, Nankoku 783-8505, Japan.

Received: 22 June 2018 Accepted: 22 June 2018

Published online: 13 August 2018

\section{References}

1. Bellomo R, Ronco C, Kellum JA, Mehta RL, Palevsky P. Acute renal failure-definition, outcome measures, animal models, fluid therapy and information technology needs: the Second International Consensus Conference of the Acute Dialysis Quality Initiative (ADQI) Group. Crit Care. 2004;8:R204-12.

2. Mehta RL, Kellum JA, Shah SV, Molitoris BA, Ronco C, Warnock DG, et al. Acute Kidney Injury Network: report of an initiative to improve outcomes in acute kidney injury. Crit Care. 2007;11:R31.

3. KDIGO. Clinical Practice Guideline for Acute Kidney Injury. Kidney Int Suppl. 2012:2:1-138.

4. Kellum JA, Levin N, Bouman C, Lameire N. Developing a consensus classification system for acute renal failure. Curr Opin Crit Care. 2002:8:509-14.

5. Lassnigg A, Schmidlin D, Mouhieddine M, Bachmann LM, Druml W, Bauer P, et al. Minimal changes of serum creatinine predict prognosis in patients after cardiothoracic surgery: a prospective cohort study. J Am Soc Nephrol. 2004;15:1597-605

6. Chertow GM, Burdick E, Honour M, Bonventre JV, Bates DW. Acute kidney injury, mortality, length of stay, and costs in hospitalized patients. J Am Soc Nephrol. 2005;16:3365-70.

7. Luo $X$, Jiang $L, D u B$, Wen $Y$, Wang $M, X i X$. A comparison of different diagnostic criteria of acute kidney injury in critically ill patients. Crit Care. 2014;18:R144.

8. Li Z, Cai L, Liang X, Du Z, Chen Y, An S, et al. Identification and predicting short-term prognosis of early cardiorenal syndrome type 1: KDIGO is superior to RIFLE or AKIN. PLoS One. 2014;9:e1 14369.

9. Rodrigues FB, Bruetto RG, Torres US, Otaviano AP, Zanetta DM, Burdmann EA. Incidence and mortality of acute kidney injury after myocardial 
infarction: a comparison between KDIGO and RIFLE criteria. PLoS One. 2013; 8:e69998.

10. Fujii T, Uchino S, Takinami M, Bellomo R. Validation of the Kidney Disease Improving Global Outcomes criteria for AKI and comparison of three criteria in hospitalized patients. Clin J Am Soc Nephrol. 2014;9:848-54.

11. Zeng X, McMahon GM, Brunelli SM, Bates DW, Waikar SS. Incidence, outcomes, and comparisons across definitions of AKI in hospitalized individuals. Clin J Am Soc Nephrol. 2014;9:12-20.

12. Levi TM, de Souza SP, de Magalhaes JG, de Carvalho MS, Cunha AL, Dantas JG, et al. Comparison of the RIFLE, AKIN and KDIGO criteria to predict mortality in critically ill patients. Rev Bras Ter Intensiva. 2013;25:290-6.

13. Shinjo H, Sato W, Imai E, Kosugi T, Hayashi H, Nishimura K, et al. Comparison of kidney disease: improving global outcomes and acute kidney injury network criteria for assessing patients in intensive care units. Clin Exp Nephrol. 2014;18:737-45.

14. Nisula S, Kaukonen KM, Vaara ST, Korhonen AM, Poukkanen M, Karlsson S, et al. Incidence, risk factors and 90-day mortality of patients with acute kidney injury in Finnish intensive care units: the FINNAKI study. Intensive Care Med. 2013;39:420-8

15. Roy AK, Mc Gorrian C, Treacy C, Kavanaugh E, Brennan A, Mahon NG, et al, A comparison of traditional and novel definitions (RIFLE, AKIN, and KDIGO) of acute kidney injury for the prediction of outcomes in acute decompensated heart failure. Cardiorenal Med. 2013;3:26-37.

16. Bastin AJ, Ostermann M, Slack AJ, Diller GP, Finney SJ, Evans TW. Acute kidney injury after cardiac surgery according to risk/injury/failure/loss/endstage, Acute Kidney Injury Network, and Kidney Disease: Improving Global Outcomes classifications. J Crit Care. 2013;28:389-96.

17. Peng $Q$, Zhang $L$, Ai Y. Epidemiology of acute kidney injury in intensive care septic patients based on the KDIGO guidelines. Chin Med J. 2014;127:1820-6.

18. Rosi S, Piano S, Frigo AC, Morando F, Fasolato S, Cavallin M, et al. New ICA criteria for the diagnosis of acute kidney injury in cirrhotic patients: can we use an imputed value of serum creatinine? Liver Int. 2015;35:2108-14.

19. Siew ED, Peterson JF, Eden SK, Moons KG, Ikizler TA, Matheny ME. Use of multiple imputation method to improve estimation of missing baseline serum creatinine in acute kidney injury research. Clin J Am Soc Nephrol. 2013;8:10-8.

20. Sims AJ, Hussein HK, Prabhu M, Kanagasundaram NS. Are surrogate assumptions and use of diuretics associated with diagnosis and staging of acute kidney injury after cardiac surgery? Clin J Am Soc Nephrol. 2012;7:15-23.

21. Candela-Toha AM, Recio-Vazquez M, Delgado-Montero A, del Rey JM, Muriel A, Liano F, et al. The calculation of baseline serum creatinine overestimates the diagnosis of acute kidney injury in patients undergoing cardiac surgery. Nefrologia. 2012:32:53-8.

22. Siew ED, Matheny ME, Ikizler TA, Lewis JB, Miller RA, Waitman LR, et al. Commonly used surrogates for baseline renal function affect the classification and prognosis of acute kidney injury. Kidney Int. 2010;77:536-42.

23. Pickering JW, Endre ZH. Back-calculating baseline creatinine with MDRD misclassifies acute kidney injury in the intensive care unit. Clin J Am Soc Nephrol. 2010;5:1165-73.

24. Bagshaw SM, Uchino S, Cruz D, Bellomo R, Morimatsu H, Morgera S, et al. A comparison of observed versus estimated baseline creatinine for determination of RIFLE class in patients with acute kidney injury. Nephrol Dial Transplant. 2009;24:2739-44.

25. Zavada J, Hoste E, Cartin-Ceba R, Calzavacca P, Gajic O, Clermont G, et al. A comparison of three methods to estimate baseline creatinine for RIFLE classification. Nephrol Dial Transplant. 2010;25:3911-8.

26. Harris SK, Lewington AJ, Harrison DA, Rowan KM. Relationship between patients' outcomes and the changes in serum creatinine and urine output and RIFLE classification in a large critical care cohort database. Kidney Int. 2015;88:369-77.

27. Kellum JA, Sileanu FE, Murugan R, Lucko N, Shaw AD, Clermont G. Classifying AKI by urine output versus serum creatinine level. J Am Soc Nephrol. 2015;26:2231-8.

28. Leedahl DD, Frazee EN, Schramm GE, Dierkhising RA, Bergstralh EJ, Chawla $L S$, et al. Derivation of urine output thresholds that identify a very high risk of AKI in patients with septic shock. Clin J Am Soc Nephrol. 2014;9:1168-74.

29. Wlodzimirow KA, Abu-Hanna A, Slabbekoorn M, Chamuleau RA, Schultz MJ, Bouman CS. A comparison of RIFLE with and without urine output criteria for acute kidney injury in critically ill patients. Crit Care. 2012;16:R200.
30. Han SS, Kang KJ, Kwon SJ, Wang SJ, Shin SH, Oh SW, et al. Additional role of urine output criterion in defining acute kidney injury. Nephrol Dial Transplant. 2012;27:161-5.

31. Macedo E, Malhotra R, Claure-Del Granado R, Fedullo P, Mehta RL. Defining urine output criterion for acute kidney injury in critically ill patients. Nephrol Dial Transplant. 2011;26:509-15.

32. Lagny MG, Jouret F, Koch JN, Blaffart F, Donneau AF, Albert A, et al. Incidence and outcomes of acute kidney injury after cardiac surgery using either criteria of the RIFLE classification. BMC Nephrol. 2015;16:76.

33. Vaara ST, Parviainen I, Pettila V, Nisula S, Inkinen O, Uusaro A. Association of oliguria with the development of acute kidney injury in the critically ill. Kidney Int. 2016;89:200-8.

34. Uchino S, Kellum JA, Bellomo R, Doig GS, Morimatsu H, Morgera S, et al. Acute renal failure in critically ill patients: a multinational, multicenter study. JAMA. 2005;294:813-8.

35. Hu J, Chen R, Liu S, Yu X, Zou J, Ding X. Global incidence and outcomes of adult patients with acute kidney injury after cardiac surgery: a systematic review and meta-analysis. J Cardiothorac Vasc Anesth. 2016;30:82-9.

36. Ozkaynak B, Kayalar N, Gumus F, Yucel C, Mert B, Boyacioglu K, et al. Time from cardiac catheterization to cardiac surgery: a risk factor for acute kidney injury? Interact Cardiovasc Thorac Surg. 2014;18:706-11.

37. Kristovic D, Horvatic I, Husedzinovic I, Sutlic Z, Rudez I, Baric D, et al. Cardiac surgery-associated acute kidney injury: risk factors analysis and comparison of prediction models. Interact Cardiovasc Thorac Surg. 2015;21:366-73.

38. Joung KW, Jo JY, Kim WJ, Choi DK, Chin JH, Lee EH, et al. Association of preoperative uric acid and acute kidney injury following cardiovascular surgery. J Cardiothorac Vasc Anesth. 2014;28:1440-7.

39. Ng RR, Chew ST, Liu W, Shen L, Ti LK. Identification of modifiable risk factors for acute kidney injury after coronary artery bypass graft surgery in an Asian population. J Thorac Cardiovasc Surg. 2014;147:1356-61.

40. Parolari A, Pesce LL, Pacini D, Mazzanti V, Salis S, Sciacovelli C, et al. Risk factors for perioperative acute kidney injury after adult cardiac surgery: role of perioperative management. Ann Thorac Surg. 2012;93:584-91.

41. Huang TM, Wu VC, Young GH, Lin YF, Shiao CC, Wu PC, et al. Preoperative proteinuria predicts adverse renal outcomes after coronary artery bypass grafting. J Am Soc Nephrol. 2011;22:156-63.

42. Guenancia C, Kahli A, Laurent G, Hachet O, Malapert G, Grosjean S, et al. Pre-operative growth differentiation factor 15 as a novel biomarker of acute kidney injury after cardiac bypass surgery. Int J Cardiol. 2015;197:66-71.

43. Kumar AB, Suneja M, Bayman EO, Weide GD, Tarasi M. Association between postoperative acute kidney injury and duration of cardiopulmonary bypass: a meta-analysis. J Cardiothorac Vasc Anesth. 2012;26:64-9.

44. Seabra VF, Alobaidi S, Balk EM, Poon AH, Jaber BL. Off-pump coronary artery bypass surgery and acute kidney injury: a meta-analysis of randomized controlled trials. Clin J Am Soc Nephrol. 2010;5:1734-44.

45. Elhmidi Y, Bleiziffer S, Deutsch MA, Krane M, Mazzitelli D, Lange R, et al. Acute kidney injury after transcatheter aortic valve implantation: incidence, predictors and impact on mortality. Arch Cardiovasc Dis. 2014;107:133-9.

46. Sampaio MS, Martin P, Bunnapradist S. Renal dysfunction in end-stage liver disease and post-liver transplant. Clin Liver Dis. 2014;18:543-60.

47. Matuszkiewicz-Rowinska J, Wieliczko M, Malyszko J. Renal replacement therapy before, during, and after orthotopic liver transplantation. Ann Transplant. 2013;18:248-55.

48. Leithead JA, Armstrong MJ, Corbett C, Andrew M, Kothari C, Gunson BK, et al. Hepatic ischemia reperfusion injury is associated with acute kidney injury following donation after brain death liver transplantation. Transpl Int. 2013; 26:1116-25.

49. Khosravi MB, Milani S, Kakaei F. Serum neutrophil gelatinase-associated lipocalin versus serum creatinine for the prediction of acute kidney injury after liver transplantation. Int J Organ Transplant Med. 2013;4:102-9.

50. Papadopoulos S, Karapanagiotou A, Kydona C, Dimitriadis C, Theodoridou T, Piperidou $\mathrm{M}$, et al. Causes and incidence of renal replacement therapy application in orthotopic liver transplantation patients: our experience. Transplant Proc. 2014;46:3228-31.

51. Leithead JA, Rajoriya N, Gunson BK, Muiesan P, Ferguson JW. The evolving use of higher risk grafts is associated with an increased incidence of acute kidney injury after liver transplantation. J Hepatol. 2014;60:1180-6.

52. Kim JM, Jo YY, Na SW, Kim Sl, Choi YS, Kim NO, et al. The predictors for continuous renal replacement therapy in liver transplant recipients. Transplant Proc. 2014;46:184-91. 
53. Chen HP, Tsai YF, Lin JR, Liu FC, Yu HP. Incidence and outcomes of acute renal failure following liver transplantation: a population-based cohort study. Medicine (Baltimore). 2015;94:e2320.

54. Kamath PS, Wiesner RH, Malinchoc M, Kremers W, Therneau TM, Kosberg CL, et al. A model to predict survival in patients with end-stage liver disease. Hepatology. 2001;33:464-70.

55. Wagener G, Minhaz M, Mattis FA, Kim M, Emond JC, Lee HT. Urinary neutrophil gelatinase-associated lipocalin as a marker of acute kidney injury after orthotopic liver transplantation. Nephrol Dial Transplant. 2011;26:1717-23.

56. Chen J, Singhapricha T, Hu KQ, Hong JC, Steadman RH, Busuttil RW, et al. Postliver transplant acute renal injury and failure by the RIFLE criteria in patients with normal pretransplant serum creatinine concentrations: a matched study. Transplantation. 2011;91:348-53.

57. Lebron Gallardo M, Herrera Gutierrez ME, Seller Perez G, Curiel Balsera E, Fernandez Ortega JF, Quesada Garcia G. Risk factors for renal dysfunction in the postoperative course of liver transplant. Liver Transpl. 2004;10:1379-85.

58. Cabezuelo JB, Ramirez P, Rios A, Acosta F, Torres D, Sansano T, et al. Risk factors of acute renal failure after liver transplantation. Kidney Int. 2006;69: 1073-80.

59. Tomozawa A, Ishikawa S, Shiota N, Cholvisudhi P, Makita K. Perioperative risk factors for acute kidney injury after liver resection surgery: an historical cohort study. Can J Anaesth. 2015;62:753-61.

60. Xue J, Wang L, Chen CM, Chen JY, Sun ZX. Acute kidney injury influences mortality in lung transplantation. Ren Fail. 2014;36:541-5.

61. George TJ, Arnaoutakis GJ, Beaty CA, Pipeling MR, Merlo CA, Conte JV, et al. Acute kidney injury increases mortality after lung transplantation. Ann Thorac Surg. 2012;94:185-92.

62. Licker M, Cartier V, Robert J, Diaper J, Villiger Y, Tschopp JM, et al. Risk factors of acute kidney injury according to RIFLE criteria after lung cancer surgery. Ann Thorac Surg. 2011;91:844-50.

63. Thakar CV, Kharat V, Blanck S, Leonard AC. Acute kidney injury after gastric bypass surgery. Clin J Am Soc Nephrol. 2007;2:426-30.

64. Morgan DJ, Ho KM. Acute kidney injury in bariatric surgery patients requiring intensive care admission: a state-wide, multicenter, cohort study. Surg Obes Relat Dis. 2015;11:1300-6.

65. Weingarten TN, Gurrieri C, McCaffrey JM, Ricter SJ, Hilgeman ML, Schroeder DR, et al. Acute kidney injury following bariatric surgery. Obes Surg. 2013;23: 64-70.

66. Causey MW, Maykel JA, Hatch Q, Miller S, Steele SR. Identifying risk factors for renal failure and myocardial infarction following colorectal surgery. J Surg Res. 2011;170:32-7.

67. Bagshaw SM, Cruz DN, Aspromonte N, Daliento L, Ronco F, Sheinfeld G, et al. Epidemiology of cardio-renal syndromes: workgroup statements from the 7th ADQI Consensus Conference. Nephrol Dial Transplant. 2010;25:1406-16.

68. Ronco C, McCullough P, Anker SD, Anand I, Aspromonte N, Bagshaw SM, et al. Cardio-renal syndromes: report from the consensus conference of the acute dialysis quality initiative. Eur Heart J. 2010;31:703-11.

69. Ronco C, House AA, Haapio M. Cardiorenal syndrome: refining the definition of a complex symbiosis gone wrong. Intensive Care Med. 2008;34:957-62.

70. Amin AP, Spertus JA, Reid KJ, Lan X, Buchanan DM, Decker C, et al. The prognostic importance of worsening renal function during an acute myocardial infarction on long-term mortality. Am Heart J. 2010;160:1065-71.

71. Wang YN, Cheng H, Yue T, Chen YP. Derivation and validation of a prediction score for acute kidney injury in patients hospitalized with acute heart failure in a Chinese cohort. Nephrology (Carlton). 2013;18:489-96.

72. Zhou Q, Zhao C, Xie D, Xu D, Bin J, Chen P, et al. Acute and acute-onchronic kidney injury of patients with decompensated heart failure: impact on outcomes. BMC Nephrol. 2012;13:51.

73. Soyler C, Tanriover MD, Ascioglu S, Aksu NM, Arici M. Urine neutrophil gelatinase-associated lipocalin levels predict acute kidney injury in acute decompensated heart failure patients. Ren Fail. 2015;37:772-6.

74. Tung YC, Chang CH, Chen YC, Chu PH. Combined biomarker analysis for risk of acute kidney injury in patients with ST-segment elevation myocardial infarction. PLoS One. 2015:10:e0125282.

75. Aghel A, Shrestha K, Mullens W, Borowski A, Tang WH. Serum neutrophil gelatinase-associated lipocalin (NGAL) in predicting worsening renal function in acute decompensated heart failure. J Card Fail. 2010;16:49-54.

76. Belziti CA, Bagnati R, Ledesma P, Vulcano N, Fernandez S. Worsening renal function in patients admitted with acute decompensated heart failure: incidence, risk factors and prognostic implications. Rev Esp Cardiol. 2010;63: 294-302.
77. Suh SH, Kim CS, Choi JS, Bae EH, Ma SK, Kim SW. Acute kidney injury in patients with sepsis and septic shock: risk factors and clinical outcomes. Yonsei Med J. 2013;54:965-72.

78. Bagshaw SM, George C, Bellomo R. Early acute kidney injury and sepsis: a multicentre evaluation. Crit Care. 2008;12:R47.

79. Poukkanen M, Wilkman E, Vaara ST, Pettila V, Kaukonen KM, Korhonen AM, et al. Hemodynamic variables and progression of acute kidney injury in critically ill patients with severe sepsis: data from the prospective observational FINNAKI study. Crit Care. 2013;17:R295.

80. Plataki M, Kashani K, Cabello-Garza J, Maldonado F, Kashyap R, Kor DJ, et al. Predictors of acute kidney injury in septic shock patients: an observational cohort study. Clin J Am Soc Nephrol. 2011;6:1744-51.

81. Medeiros P, Nga HS, Menezes P, Bridi R, Balbi A, Ponce D. Acute kidney injury in septic patients admitted to emergency clinical room: risk factors and outcome. Clin Exp Nephrol. 2015;19:859-66.

82. Legrand M, Dupuis C, Simon C, Gayat E, Mateo J, Lukaszewicz AC, et al. Association between systemic hemodynamics and septic acute kidney injury in critically ill patients: a retrospective observational study. Crit Care. 2013;17:R278.

83. Chang CW, Kok VC, Tseng TC, Horng JT, Liu CE. Diabetic patients with severe sepsis admitted to intensive care unit do not fare worse than nondiabetic patients: a nationwide population-based cohort study. PLoS One. 2012;7:e50729.

84. Venot M, Weis L, Clec'h C, Darmon M, Allaouchiche B, Goldgran-Toledano D, et al. Acute kidney injury in severe sepsis and septic shock in patients with and without diabetes mellitus: a multicenter study. PLoS One. 2015;10: e0127411.

85. Nash K, Hafeez A, Hou S. Hospital-acquired renal insufficiency. Am J Kidney Dis. 2002;39:930-6.

86. Mesropian PD, Othersen J, Mason D, Wang J, Asif A, Mathew RO. Community-acquired acute kidney injury: a challenge and opportunity for primary care in kidney health. Nephrology (Carlton). 2016;21:729-35.

87. Mehta RL, Cerda J, Burdmann EA, Tonelli M, Garcia-Garcia G, Jha V, et al. International Society of Nephrology's 0 by 25 initiative for acute kidney injury (zero preventable deaths by 2025): a human rights case for nephrology. Lancet. 2015:385:2616-43.

88. Loo CS, Zainal D. Acute renal failure in a teaching hospital. Singap Med J. 1995:36:278-81.

89. Obialo Cl, Okonofua EC, Tayade AS, Riley LJ. Epidemiology of de novo acute renal failure in hospitalized African Americans: comparing communityacquired vs hospital-acquired disease. Arch Intern Med. 2000;160:1309-13.

90. Sesso R, Roque A, Vicioso B, Stella S. Prognosis of ARF in hospitalized elderly patients. Am J Kidney Dis. 2004;44:410-9.

91. Wang Y, Cui Z, Fan M. Hospital-acquired and community-acquired acute renal failure in hospitalized Chinese: a ten-year review. Ren Fail. 2007;29:163-8.

92. Schissler MM, Zaidi S, Kumar H, Deo D, Brier ME, McLeish KR. Characteristics and outcomes in community-acquired versus hospital-acquired acute kidney injury. Nephrology (Carlton). 2013;18:183-7.

93. Der Mesropian PJ, Kalamaras JS, Eisele G, Phelps KR, Asif A, Mathew RO. Long-term outcomes of community-acquired versus hospital-acquired acute kidney injury: a retrospective analysis. Clin Nephrol. 2014;81:174-84.

94. Wonnacott A, Meran S, Amphlett B, Talabani B, Phillips A. Epidemiology and outcomes in community-acquired versus hospital-acquired AKI. Clin J Am Soc Nephrol. 2014;9:1007-14.

95. Xu X, Nie S, Liu Z, Chen C, Xu G, Zha Y, et al. Epidemiology and clinical correlates of AKI in Chinese hospitalized adults. Clin J Am Soc Nephrol. 2015;10:1510-8.

96. Hoste EA, Bagshaw SM, Bellomo R, Cely CM, Colman R, Cruz DN, et al. Epidemiology of acute kidney injury in critically ill patients: the multinational AKI-EPI study. Intensive Care Med. 2015:41:1411-23.

97. Iwagami M, Yasunaga H, Noiri E, Horiguchi H, Fushimi K, Matsubara T, et al. Current state of continuous renal replacement therapy for acute kidney injury in Japanese intensive care units in 2011: analysis of a national administrative database. Nephrol Dial Transplant. 2015;30:988-95.

98. Neveu $H$, Kleinknecht D, Brivet F, Loirat $P$, Landais P. Prognostic factors in acute renal failure due to sepsis. Results of a prospective multicentre study. The French Study Group on acute renal failure. Nephrol Dial Transplant. 1996:11:293-9.

99. Bagshaw SM, Uchino S, Bellomo R, Morimatsu H, Morgera S, Schetz M, et al. Septic acute kidney injury in critically ill patients: clinical characteristics and outcomes. Clin J Am Soc Nephrol. 2007;2:431-9. 
100. Bagshaw SM, Bennett M, Devarajan P, Bellomo R. Urine biochemistry in septic and non-septic acute kidney injury: a prospective observational study. J Crit Care. 2013;28:371-8.

101. Nagata I, Uchino S, Tokuhira N, Ohnuma T, Namba Y, Katayama S, et al. Sepsis may not be a risk factor for mortality in patients with acute kidney injury treated with continuous renal replacement therapy. J Crit Care. 2015; 30:998-1002.

102. Hamzic-Mehmedbasic A, Rasic S, Rebic D, Durak-Nalbantic A, Muslimovic A, Dzemidzic J. Renal function outcome prognosis in septic and non-septic acute kidney injury patients. Med Arch. 2015;69:77-80.

103. Cruz MG, Dantas JG, Levi TM, Rocha Mde S, de Souza SP, Boa-Sorte N, et al. Septic versus non-septic acute kidney injury in critically ill patients: characteristics and clinical outcomes. Rev Bras Ter Intensiva. 2014;26:384-91.

104. Carbonell N, Blasco M, Ferreres J, Blanquer J, Garcia-Ramon R, Mesejo A, et al. Sepsis and SOFA score: related outcome for critically ill renal patients. Clin Nephrol. 2004;62:185-92

105. Martensson J, Martling CR, Oldner A, Bell M. Impact of sepsis on levels of plasma cystatin C in AKI and non-AKI patients. Nephrol Dial Transplant. 2012;27:576-81

106. Himmelfarb J, Joannidis M, Molitoris B, Schietz M, Okusa MD, Warnock D, et al. Evaluation and initial management of acute kidney injury. Clin J Am Soc Nephrol. 2008;3:962-7

107. Doi K, Katagiri D, Negishi K, Hasegawa S, Hamasaki Y, Fujita T, et al. Mild elevation of urinary biomarkers in prerenal acute kidney injury. Kidney Int. 2012;82:1114-20.

108. Perinel S, Vincent F, Lautrette A, Dellamonica J, Mariat C, Zeni F, et al. Transient and persistent acute kidney injury and the risk of hospital mortality in critically ill patients: results of a multicenter cohort study. Crit Care Med. 2015;43:e269-75.

109. Yang F, Zhang L, Wu H, Zou H, Du Y. Clinical analysis of cause, treatment and prognosis in acute kidney injury patients. PLoS One. 2014;9:e85214.

110. Kaufman J, Dhakal M, Patel B, Hamburger R. Community-acquired acute renal failure. Am J Kidney Dis. 1991;17:191-8.

111. Koyner JL, Garg AX, Thiessen-Philbrook H, Coca SG, Cantley LG, Peixoto A, et al. Adjudication of etiology of acute kidney injury: experience from the TRIBE-AKI multi-center study. BMC Nephrol. 2014;15:105.

112. Nickolas TL, O'Rourke MJ, Yang J, Sise ME, Canetta PA, Barasch N, et al. Sensitivity and specificity of a single emergency department measurement of urinary neutrophil gelatinase-associated lipocalin for diagnosing acute kidney injury. Ann Intern Med. 2008;148:810-9.

113. Park MY, Choi SJ, Kim JK, Hwang SD, Lee YW. Urinary cystatin C levels as a diagnostic and prognostic biomarker in patients with acute kidney injury. Nephrology (Carlton). 2013;18:256-62.

114. Santacruz F, Barreto S, Mayor MM, Cabrera W, Breuer N. Mortality in elderly patients with acute renal failure. Ren Fail. 1996;18:601-5.

115. Singer E, Elger A, Elitok S, Kettritz R, Nickolas TL, Barasch J, et al. Urinary neutrophil gelatinase-associated lipocalin distinguishes pre-renal from intrinsic renal failure and predicts outcomes. Kidney Int. 2011;80:405-14.

116. Soto K, Coelho S, Rodrigues B, Martins H, Frade F, Lopes S, et al. Cystatin C as a marker of acute kidney injury in the emergency department. Clin J Am Soc Nephrol. 2010;5:1745-54

117. Ho J, Tangri N, Komenda P, Kaushal A, Sood M, Brar R, et al. Urinary, plasma, and serum biomarkers' utility for predicting acute kidney injury associated with cardiac surgery in adults: a meta-analysis. Am J Kidney Dis. 2015;66: 993-1005.

118. Parikh CR, Lu JC, Coca SG, Devarajan P. Tubular proteinuria in acute kidney injury: a critical evaluation of current status and future promise. Ann Clin Biochem. 2010;47:301-12.

119. Coca SG, Yalavarthy R, Concato J, Parikh CR. Biomarkers for the diagnosis and risk stratification of acute kidney injury: a systematic review. Kidney Int. 2008;73:1008-16.

120. Haase-Fielitz A, Haase M, Devarajan P. Neutrophil gelatinase-associated lipocalin as a biomarker of acute kidney injury: a critical evaluation of current status. Ann Clin Biochem. 2014:51:335-51.

121. Hjortrup PB, Haase N, Wetterslev M, Perner A. Clinical review: predictive value of neutrophil gelatinase-associated lipocalin for acute kidney injury in intensive care patients. Crit Care. 2013;17:211.

122. Haase M, Bellomo R, Devarajan P, Schlattmann P, Haase-Fielitz A. Accuracy of neutrophil gelatinase-associated lipocalin (NGAL) in diagnosis and prognosis in acute kidney injury: a systematic review and meta-analysis. Am J Kidney Dis. 2009;54:1012-24.
123. Koyner JL, Bennett MR, Worcester EM, Ma Q, Raman J, Jeevanandam V, et al. Urinary cystatin $\mathrm{C}$ as an early biomarker of acute kidney injury following adult cardiothoracic surgery. Kidney Int. 2008;74:1059-69.

124. Tuladhar SM, Puntmann VO, Soni M, Punjabi PP, Bogle RG. Rapid detection of acute kidney injury by plasma and urinary neutrophil gelatinaseassociated lipocalin after cardiopulmonary bypass. J Cardiovasc Pharmacol. 2009;53:261-6.

125. de Geus HR, Bakker J, Lesaffre EM, le Noble JL. Neutrophil gelatinaseassociated lipocalin at ICU admission predicts for acute kidney injury in adult patients. Am J Respir Crit Care Med. 2011;183:907-14.

126. Wagener G, Jan M, Kim M, Mori K, Barasch JM, Sladen RN, et al. Association between increases in urinary neutrophil gelatinase-associated lipocalin and acute renal dysfunction after adult cardiac surgery. Anesthesiology. 2006; 105:485-91.

127. Wagener G, Gubitosa G, Wang S, Borregaard N, Kim M, Lee HT. Urinary neutrophil gelatinase-associated lipocalin and acute kidney injury after cardiac surgery. Am J Kidney Dis. 2008;52:425-33.

128. Xin C, Yulong X, Yu C, Changchun C, Feng Z, Xinwei M. Urine neutrophil gelatinase-associated lipocalin and interleukin-18 predict acute kidney injury after cardiac surgery. Ren Fail. 2008;30:904-13.

129. Han WK, Wagener G, Zhu Y, Wang S, Lee HT. Urinary biomarkers in the early detection of acute kidney injury after cardiac surgery. Clin J Am Soc Nephrol. 2009;4:873-82.

130. Liangos O, Tighiouart H, Perianayagam MC, Kolyada A, Han WK, Wald R, et al. Comparative analysis of urinary biomarkers for early detection of acute kidney injury following cardiopulmonary bypass. Biomarkers. 2009; 14:423-31.

131. Makris K, Markou N, Evodia E, Dimopoulou E, Drakopoulos I, Ntetsika K, et al. Urinary neutrophil gelatinase-associated lipocalin (NGAL) as an early marker of acute kidney injury in critically ill multiple trauma patients. Clin Chem Lab Med. 2009;47:79-82.

132. Heise D, Rentsch K, Braeuer A, Friedrich M, Quintel M. Comparison of urinary neutrophil glucosaminidase-associated lipocalin, cystatin C, and alpha1microglobulin for early detection of acute renal injury after cardiac surgery. Eur J Cardiothorac Surg. 2011;39:38-43.

133. Ejaz AA, Kambhampati G, Ejaz NI, Dass B, Lapsia V, Arif AA, et al. Postoperative serum uric acid and acute kidney injury. J Nephrol. 2012;25: 497-505.

134. Sargentini $V$, Mariani P, D'Alessandro M, Pistolesi $V$, Lauretta MP, Pacini F, et al. Assessment of NGAL as an early biomarker of acute kidney injury in adult cardiac surgery patients. J Biol Regul Homeost Agents. 2012;26:485-93.

135. Liebetrau C, Dorr O, Baumgarten H, Gaede L, Szardien S, Blumenstein J, et al. Neutrophil gelatinase-associated lipocalin (NGAL) for the early detection of cardiac surgery associated acute kidney injury. Scand J Clin Lab Invest. 2013:73:392-9.

136. Liu S, Che M, Xue S, Xie B, Zhu M, Lu R, et al. Urinary L-FABP and its combination with urinary NGAL in early diagnosis of acute kidney injury after cardiac surgery in adult patients. Biomarkers. 2013;18:95-101.

137. Munir MU, Khan DA, Khan FA, Shahab Naqvi SM. Rapid detection of acute kidney injury by urinary neutrophil gelatinase-associated lipocalin after cardiopulmonary bypass surgery. J Coll Physicians Surg Pak. 2013;23:103-6.

138. Paarmann H, Charitos El, Beilharz A, Heinze H, Schön J, Berggreen A, et al. Duration of cardiopulmonary bypass is an important confounder when using biomarkers for early diagnosis of acute kidney injury in cardiac surgical patients. Appl Cardiopulm Pathophysiol. 2013;17:284-97.

139. Schinstock CA, Semret MH, Wagner SJ, Borland TM, Bryant SC, Kashani KB, et al. Urinalysis is more specific and urinary neutrophil gelatinase-associated lipocalin is more sensitive for early detection of acute kidney injury. Nephrol Dial Transplant. 2013;28:1175-85.

140. Susantitaphong P, Siribamrungwong M, Doi K, Noiri E, Terrin N, Jaber BL. Performance of urinary liver-type fatty acid-binding protein in acute kidney injury: a meta-analysis. Am J Kidney Dis. 2013;61:430-9.

141. Matsui K, Kamijo-Ikemori A, Sugaya T, Yasuda T, Kimura K. Usefulness of urinary biomarkers in early detection of acute kidney injury after cardiac surgery in adults. Circ J. 2012;76:213-20.

142. Doi K, Negishi K, Ishizu T, Katagiri D, Fujita T, Matsubara T, et al. Evaluation of new acute kidney injury biomarkers in a mixed intensive care unit. Crit Care Med. 2011;39:2464-9.

143. Matsui K, Kamijo-Ikemori A, Hara M, Sugaya T, Kodama T, Fujitani S, et al. Clinical significance of tubular and podocyte biomarkers in acute kidney injury. Clin Exp Nephrol. 2011;15:220-5. 
144. Cho E, Yang HN, Jo SK, Cho WY, Kim HK. The role of urinary liver-type fatty acid-binding protein in critically ill patients. J Korean Med Sci. 2013;28:100-5

145. Nickolas TL, Schmidt-Ott KM, Canetta P, Forster C, Singer E, Sise M, et al. Diagnostic and prognostic stratification in the emergency department using urinary biomarkers of nephron damage: a multicenter prospective cohort study. J Am Coll Cardiol. 2012;59:246-55.

146. Katagiri D, Doi K, Honda K, Negishi K, Fujita T, Hisagi M, et al. Combination of two urinary biomarkers predicts acute kidney injury after adult cardiac surgery. Ann Thorac Surg. 2012;93:577-83.

147. Zhang Z, Lu B, Sheng X, Jin N. Cystatin C in prediction of acute kidney injury: a systemic review and meta-analysis. Am J Kidney Dis. 2011;58:356-65.

148. Nejat M, Pickering JW, Walker RJ, Westhuyzen J, Shaw GM, Frampton CM, et al. Urinary cystatin $C$ is diagnostic of acute kidney injury and sepsis, and predicts mortality in the intensive care unit. Crit Care. 2010;14:R85.

149. Koyner JL, Vaidya VS, Bennett MR, Ma Q, Worcester E, Akhter SA, et al. Urinary biomarkers in the clinical prognosis and early detection of acute kidney injury. Clin J Am Soc Nephrol. 2010;5:2154-65.

150. Ware LB, Johnson AC, Zager RA. Renal cortical albumin gene induction and urinary albumin excretion in response to acute kidney injury. Am J Physiol Renal Physiol. 2011;300:F628-38.

151. McCullough PA, Jefferies JL. Novel markers and therapies for patients with acute heart failure and renal dysfunction. Am J Med. 2015;128:312. e1-22

152. Feng Y, Zhang Y, Li G, Wang L. Relationship of cystatin-C change and the prevalence of death or dialysis need after acute kidney injury: a metaanalysis. Nephrology (Carlton). 2014;19:679-84.

153. Seibert FS, Pagonas N, Arndt R, Heller F, Dragun D, Persson P, et al. Calprotectin and neutrophil gelatinase-associated lipocalin in the differentiation of pre-renal and intrinsic acute kidney injury. Acta Physiol (Oxf). 2013;207:700-8.

154. Jayaraman R, Sunder S, Sathi S, Gupta VK, Sharma N, Kanchi P, et al. Post cardiac surgery acute kidney injury: a woebegone status rejuvenated by the novel biomarkers. Nephrourol Mon. 2014;6:e19598.

155. Nejat M, Pickering JW, Devarajan P, Bonventre JV, Edelstein CL, Walker RJ, et al. Some biomarkers of acute kidney injury are increased in pre-renal acute injury. Kidney Int. 2012;81:1254-62.

156. Nigwekar SU, Navaneethan SD, Parikh CR, Hix JK. Atrial natriuretic peptide for preventing and treating acute kidney injury. Cochrane Database Syst Rev. 2009:CD006028

157. Zacharias M, Mugawar M, Herbison GP, Walker RJ, Hovhannisyan K, Sivalingam $P$, et al. Interventions for protecting renal function in the perioperative period. Cochrane Database Syst Rev. 2013:CD003590.

158. Sezai A, Hata M, Niino T, Yoshitake I, Unosawa S, Wakui S, et al. Influence of continuous infusion of low-dose human atrial natriuretic peptide on renal function during cardiac surgery: a randomized controlled study. J Am Coll Cardiol. 2009;54:1058-64.

159. Sezai A, Hata M, Niino T, Yoshitake I, Unosawa S, Wakui S, et al. Results of low-dose human atrial natriuretic peptide infusion in nondialysis patients with chronic kidney disease undergoing coronary artery bypass grafting: the NU-HIT (Nihon University working group study of low-dose HANP infusion therapy during cardiac surgery) trial for CKD. J Am Coll Cardiol. 2011;58:897-903.

160. Kangawa K, Matsuo H. Purification and complete amino acid sequence of alpha-human atrial natriuretic polypeptide (alpha-hANP). Biochem Biophys Res Commun. 1984:118:131-9.

161. Nakao K, Ogawa Y, Suga S, Imura H. Molecular biology and biochemistry of the natriuretic peptide system. I: natriuretic peptides. J Hypertens. 1992;10:907-12.

162. Mukoyama M, Nakao K, Hosoda K, Suga S, Saito Y, Ogawa Y, et al. Brain natriuretic peptide as a novel cardiac hormone in humans. Evidence for an exquisite dual natriuretic peptide system, atrial natriuretic peptide and brain natriuretic peptide. J Clin Invest. 1991;87:1402-12.

163. Yasue H, Yoshimura M, Sumida H, Kikuta K, Kugiyama K, Jougasaki M, et al. Localization and mechanism of secretion of B-type natriuretic peptide in comparison with those of A-type natriuretic peptide in normal subjects and patients with heart failure. Circulation. 1994;90:195-203.

164. Yoshimura M, Yasue $H$, Ogawa $H$. Pathophysiological significance and clinical application of ANP and BNP in patients with heart failure. Can J Physiol Pharmacol. 2001;79:730-5.

165. Yoshimura M, Yasue $H$, Morita E, Sakaino N, Jougasaki M, Kurose M, et al. Hemodynamic, renal, and hormonal responses to brain natriuretic peptide infusion in patients with congestive heart failure. Circulation. 1991:84:1581-8

166. Allgren RL, Marbury TC, Rahman SN, Weisberg LS, Fenves AZ, Lafayette RA et al. Auriculin Anaritide Acute Renal Failure Study Group. Anaritide in acute tubular necrosis. N Engl J Med 1997;336:828-834.

167. Lewis J, Salem MM, Chertow GM, Weisberg LS, McGrew F, Marbury TC et al. Auriculin Anaritide Acute Renal Failure Study Group. Atrial natriuretic factor in oliguric acute renal failure. Am J Kidney Dis 2000;36:767-774.

168. Sward K, Valsson F, Odencrants P, Samuelsson O, Ricksten SE. Recombinant human atrial natriuretic peptide in ischemic acute renal failure: a randomized placebo-controlled trial. Crit Care Med. 2004;32:1310-5.

169. Goetz K, Drummer C, Zhu JL, Leadley R, Fiedler F, Gerzer R. Evidence that urodilatin, rather than ANP, regulates renal sodium excretion. J Am Soc Nephrol. 1990;1:867-74.

170. Brenner P, Meyer M, Reichenspurner H, Meiser B, Muller R, Mentz P, et al. Significance of prophylactic urodilatin (INN: ularitide) infusion for the prevention of acute renal failure in patients after heart transplantation. Eur J Med Res. 1995;1:137-43.

171. Hager B, Betschart M, Krapf R. Effect of postoperative intravenous loop diuretic on renal function after major surgery. Schweiz Med Wochenschr. 1996;126:666-73.

172. Lassnigg A, Donner E, Grubhofer G, Presterl E, Druml W, Hiesmayr M. Lack of renoprotective effects of dopamine and furosemide during cardiac surgery. J Am Soc Nephrol. 2000;11:97-104.

173. Mahesh B, Yim B, Robson D, Pillai R, Ratnatunga C, Pigott D. Does furosemide prevent renal dysfunction in high-risk cardiac surgical patients? Results of a double-blinded prospective randomised trial. Eur J Cardiothorac Surg. 2008;33:370-6.

174. Ho KM, Power BM. Benefits and risks of furosemide in acute kidney injury. Anaesthesia. 2010;65:283-93.

175. Cantarovich F, Fernandez JC, Locatelli A, Perez Loredo J. Frusemide in high doses in the treatment of acute renal failure. Postgrad Med J. 1971; 47(Suppl):13-7.

176. Karayannopoulos S. High-dose frusemide in renal failure. Br Med J. 1974;2:278-9.

177. Kleinknecht D, Ganeval D, Gonzalez-Duque LA, Fermanian J. Furosemide in acute oliguric renal failure. A controlled trial. Nephron. 1976;17:51-8.

178. Brown CB, Ogg CS, Cameron JS. High dose frusemide in acute renal failure: a controlled trial. Clin Nephrol. 1981;15:90-6.

179. Shilliday IR, Quinn KJ, Allison ME. Loop diuretics in the management of acute renal failure: a prospective, double-blind, placebo-controlled, randomized study. Nephrol Dial Transplant. 1997;12:2592-6.

180. Cantarovich F, Rangoonwala B, Lorenz H, Verho M, Esnault VL. High-dose furosemide for established ARF: a prospective, randomized, double-blind, placebo-controlled, multicenter trial. Am J Kidney Dis. 2004;44:402-9.

181. van der Voort PH, Boerma EC, Koopmans M, Zandberg M, de Ruiter J, Gerritsen RT, et al. Furosemide does not improve renal recovery after hemofiltration for acute renal failure in critically ill patients: a double blind randomized controlled trial. Crit Care Med. 2009;37:533-8.

182. Ho KM, Sheridan DJ. Meta-analysis of frusemide to prevent or treat acute renal failure. BMJ. 2006;333:420

183. Yang B, Xu J, Xu F, Zou Z, Ye C, Mei C, et al. Intravascular administration of mannitol for acute kidney injury prevention: a systematic review and metaanalysis. PLoS One. 2014;9:e85029.

184. Esfahani HS, Nooraei N, Asgary M, Hashemian MR. The effect of mannitol administration to kidney donor on short-term outcomes of kidney transplantation. Saudi J Kidney Dis Transpl. 2014;25:1154-9.

185. Kalimeris K, Nikolakopoulos N, Riga M, Christodoulaki K, Moulakakis KG, Dima C, et al. Mannitol and renal dysfunction after endovascular aortic aneurysm repair procedures: a randomized trial. J Cardiothorac Vasc Anesth. 2014;28:954-9.

186. Bellomo R, Chapman M, Finfer S, Hickling K, Myburgh J. Low-dose dopamine in patients with early renal dysfunction: a placebo-controlled randomised trial. Australian and New Zealand Intensive Care Society (ANZICS) Clinical Trials Group. Lancet. 2000;356:2139-43.

187. Lauschke A, Teichgraber UK, Frei U, Eckardt KU. 'Low-dose' dopamine worsens renal perfusion in patients with acute renal failure. Kidney Int. 2006;69:1669-74

188. Friedrich JO, Adhikari N, Herridge MS, Beyene J. Meta-analysis: low-dose dopamine increases urine output but does not prevent renal dysfunction or death. Ann Intern Med. 2005;142:510-24.

189. Shah RA, Subban V, Lakshmanan A, Narayanan S, Udhayakumaran K, Pakshirajan $B$, et al. A prospective, randomized study to evaluate the 
efficacy of various diuretic strategies in acute decompensated heart failure. Indian Heart J. 2014:66:309-16.

190. Triposkiadis FK, Butler J, Karayannis G, Starling RC, Filippatos G, Wolski K, et al. Efficacy and safety of high dose versus low dose furosemide with or without dopamine infusion: the Dopamine in Acute Decompensated Heart Failure II (DAD-HF II) trial. Int J Cardiol. 2014;172:115-21.

191. Chen HH, Anstrom KJ, Givertz MM, Stevenson LW, Semigran MJ, Goldsmith $\mathrm{SR}$, et al. Low-dose dopamine or low-dose nesiritide in acute heart failure with renal dysfunction: the ROSE acute heart failure randomized trial. JAMA. 2013;310:2533-43.

192. Russo A, Bevilacqua F, Scagliusi A, Scarano A, Di Stasio E, Marana R, et al. Dopamine infusion and fluid administration improve renal function during laparoscopic surgery. Minerva Anestesiol. 2014;80:452-60.

193. Naranjo A, Cruz A, Lopez P, Chicano M, Martin-Malo A, Sitges-Serra A, et al. Renal function after dopamine and fluid administration in patients with malignant obstructive jaundice. A prospective randomized study. J Gastrointestin Liver Dis. 2011;20:161-7.

194. Cano N, Fiaccadori E, Tesinsky P, Toigo G, Druml W, Kuhlmann M, et al. ESPEN Guidelines on Enteral Nutrition: adult renal failure. Clin Nutr. 2006;25:295-310.

195. Li Y, Tang X, Zhang J, Wu T. Nutritional support for acute kidney injury. Cochrane Database Syst Rev. 2012:CD005426.

196. Marik PE, Zaloga GP. Early enteral nutrition in acutely ill patients: a systematic review. Crit Care Med. 2001;29:2264-70.

197. Heyland DK, Dhaliwal R, Drover JW, Gramlich L, Dodek P. Canadian clinical practice guidelines for nutrition support in mechanically ventilated, critically ill adult patients. JPEN J Parenter Enteral Nutr. 2003;27:355-73.

198. Doig GS, Heighes PT, Simpson F, Sweetman EA. Early enteral nutrition reduces mortality in trauma patients requiring intensive care: a metaanalysis of randomised controlled trials. Injury. 2011;42:50-6.

199. Bost RB, Tjan DH, van Zanten AR. Timing of (supplemental) parenteral nutrition in critically ill patients: a systematic review. Ann Intensive Care. 2014:4:31.

200. Casaer MP, Mesotten D, Hermans G, Wouters PJ, Schetz M, Meyfroidt G, et al. Early versus late parenteral nutrition in critically ill adults. N Engl J Med. 2011;365:506-17.

201. Dhaliwal R, Jurewitsch B, Harrietha D, Heyland DK. Combination enteral and parenteral nutrition in critically ill patients: harmful or beneficial? A systematic review of the evidence. Intensive Care Med. 2004;30:1666-71.

202. Finfer S, Liu B, Chittock DR, Norton R, Myburgh JA, McArthur C, et al. Hypoglycemia and risk of death in critically ill patients. N Engl J Med. 2012; 367:1108-18.

203. Fiaccadori E, Sabatino A, Morabito S, Bozzoli L, Donadio C, Maggiore U, et al. Hyper/hypoglycemia and acute kidney injury in critically ill patients. Clin Nutr. 2016;35:317-21.

204. Moriyama N, Ishihara M, Noguchi T, Nakanishi M, Arakawa T, Asaumi Y, et al. Admission hyperglycemia is an independent predictor of acute kidney injury in patients with acute myocardial infarction. Circ J. 2014;78:1475-80.

205. Shacham Y, Gal-Oz A, Leshem-Rubinow E, Arbel Y, Keren G, Roth A, et al. Admission glucose levels and the risk of acute kidney injury in nondiabetic ST segment elevation myocardial infarction patients undergoing primary percutaneous coronary intervention. Cardiorenal Med. 2015;5:191-8.

206. Scheinkestel CD, Kar L, Marshall K, Bailey M, Davies A, Nyulasi I, et al. Prospective randomized trial to assess caloric and protein needs of critically ill, anuric, ventilated patients requiring continuous renal replacement therapy. Nutrition. 2003;19:909-16.

207. Wooley JA, Btaiche IF, Good KL. Metabolic and nutritional aspects of acute renal failure in critically ill patients requiring continuous renal replacement therapy. Nutr Clin Pract. 2005;20:176-91.

208. Gunst J, Vanhorebeek I, Casaer MP, Hermans G, Wouters PJ, Dubois J, et al. Impact of early parenteral nutrition on metabolism and kidney injury. J Am Soc Nephrol. 2013;24:995-1005.

209. Marin A, Hardy G. Practical implications of nutritional support during continuous renal replacement therapy. Curr Opin Clin Nutr Metab Care. 2001:4:219-25.

210. Bozfakioglu S. Nutrition in patients with acute renal failure. Nephrol Dial Transplant. 2001;16(Suppl 6):21-2.

211. Zhao Y, Li Z, Shi Y, Cao G, Meng F, Zhu W, et al. Effect of hypophosphatemia on the withdrawal of mechanical ventilation in patients with acute exacerbations of chronic obstructive pulmonary disease. Biomed Rep. 2016;4:413-6.
212. Besnard N, Serveaux M, Machado S, Daubin D, Brunot V, Amigues L, et al. Electrolytes-enriched hemodiafiltration solutions for continuous renal replacement therapy in acute kidney injury: a crossover study. Blood Purif. 2016;42:18-26.

213. Karvellas CJ, Farhat MR, Sajjad I, Mogensen SS, Leung AA, Wald R, et al. A comparison of early versus late initiation of renal replacement therapy in critically ill patients with acute kidney injury: a systematic review and metaanalysis. Crit Care. 2011;15:R72.

214. Bouman CS, Oudemans-Van Straaten HM, Tijssen JG, Zandstra DF, Kesecioglu J. Effects of early high-volume continuous venovenous hemofiltration on survival and recovery of renal function in intensive care patients with acute renal failure: a prospective, randomized trial. Crit Care Med. 2002;30:2205-11.

215. Jamale TE, Hase NK, Kulkarni M, Pradeep KJ, Keskar V, Jawale S, et al. Earlier-start versus usual-start dialysis in patients with communityacquired acute kidney injury: a randomized controlled trial. Am J Kidney Dis. 2013:62:1116-21.

216. Wald R, Adhikari NK, Smith OM, Weir MA, Pope K, Cohen A, et al. Comparison of standard and accelerated initiation of renal replacement therapy in acute kidney injury. Kidney Int. 2015;88:897-904.

217. Durmaz I, Yagdi T, Calkavur T, Mahmudov R, Apaydin AZ, Posacioglu H, et al. Prophylactic dialysis in patients with renal dysfunction undergoing onpump coronary artery bypass surgery. Ann Thorac Surg. 2003;75:859-64.

218. Sugahara S, Suzuki H. Early start on continuous hemodialysis therapy improves survival rate in patients with acute renal failure following coronary bypass surgery. Hemodial Int. 2004;8:320-5.

219. Combes A, Brechot N, Amour J, Cozic N, Lebreton G, Guidon C, et al. Early high-volume hemofiltration versus standard care for post-cardiac surgery shock. The HEROICS study. Am J Respir Crit Care Med. 2015;192:1179-90.

220. Gaudry S, Hajage D, Schortgen F, Martin-Lefevre L, Pons B, Boulet E, et al. Initiation strategies for renal-replacement therapy in the intensive care unit. N Engl J Med. 2016;375:122-33.

221. Zarbock A, Kellum JA, Schmidt C, Van Aken H, Wempe C, Pavenstadt H, et al. Effect of early vs delayed initiation of renal replacement therapy on mortality in critically ill patients with acute kidney injury: the ELAIN randomized clinical trial. JAMA. 2016;315:2190-9.

222. Xu Y, Gao J, Zheng X, Zhong B, Na Y, Wei J. Timing of initiation of renal replacement therapy for acute kidney injury: a systematic review and metaanalysis of randomized-controlled trials. Clin Exp Nephrol. 2017;21:552-62.

223. Smith OM, Wald R, Adhikari NK, Pope K, Weir MA, Bagshaw SM. Standard versus accelerated initiation of renal replacement therapy in acute kidney injury (STARRT-AKI): study protocol for a randomized controlled trial. Trials. 2013;14:320.

224. Barbar SD, Binquet C, Monchi M, Bruyere R, Quenot JP. Impact on mortality of the timing of renal replacement therapy in patients with severe acute kidney injury in septic shock: the IDEAL-ICU study (initiation of dialysis early versus delayed in the intensive care unit): study protocol for a randomized controlled trial. Trials. 2014;15:270.

225. Wu VC, Ko WJ, Chang HW, Chen YW, Lin YF, Shiao CC, et al. Risk factors of early redialysis after weaning from postoperative acute renal replacement therapy. Intensive Care Med. 2008;34:101-8.

226. Kawarazaki H, Uchino S, Tokuhira N, Ohnuma T, Namba Y, Katayama S, et al. Who may not benefit from continuous renal replacement therapy in acute kidney injury? Hemodial Int. 2013;17:624-32.

227. Uchino S, Bellomo R, Morimatsu H, Morgera S, Schetz M, Tan I, et al. Discontinuation of continuous renal replacement therapy: a post hoc analysis of a prospective multicenter observational study. Crit Care Med. 2009;37:2576-82.

228. Palevsky PM, Zhang JH, O'Connor TZ, Chertow GM, Crowley ST, Choudhury D, et al. Intensity of renal support in critically ill patients with acute kidney injury. N Engl J Med. 2008;359:7-20.

229. Tolwani AJ, Campbell RC, Stofan BS, Lai KR, Oster RA, Wille KM. Standard versus high-dose CWHDF for ICU-related acute renal failure. J Am Soc Nephrol. 2008;19:1233-8.

230. Bellomo R, Cass A, Cole L, Finfer S, Gallagher M, Lo S, et al. Intensity of continuous renal-replacement therapy in critically ill patients. N Engl J Med. 2009;361:1627-38

231. Ronco C, Bellomo R, Homel P, Brendolan A, Dan M, Piccinni P, et al. Effects of different doses in continuous veno-venous haemofiltration on outcomes of acute renal failure: a prospective randomised trial. Lancet. 2000;356:26-30. 
232. Ghani RA, Zainudin S, Ctkong N, Rahman AF, Wafa SR, Mohamad M, et al. Serum IL-6 and IL-1-ra with sequential organ failure assessment scores in septic patients receiving high-volume haemofiltration and continuous venovenous haemofiltration. Nephrology (Carlton). 2006;11:386-93.

233. Boussekey N, Chiche A, Faure K, Devos P, Guery B, d'Escrivan T, et al. A pilot randomized study comparing high and low volume hemofiltration on vasopressor use in septic shock. Intensive Care Med. 2008;34:1646-53.

234. Zhang $P$, Yang Y, LV R, Zhang Y, Xie W, Chen J. Effect of the intensity of continuous renal replacement therapy in patients with sepsis and acute kidney injury: a single-center randomized clinical trial. Nephrol Dial Transplant. 2012;27:967-73.

235. Joannes-Boyau O, Honore PM, Perez P, Bagshaw SM, Grand H, Canivet JL, et al. High-volume versus standard-volume haemofiltration for septic shock patients with acute kidney injury (IVOIRE study): a multicentre randomized controlled trial. Intensive Care Med. 2013;39:1535-46.

236. Fujii T, Namba Y, Fujitani S, Sasaki J, Narihara K, Shibagaki Y, et al. Low-dose continuous renal replacement therapy for acute kidney injury. Int J Artif Organs. 2012;35:525-30.

237. Uchino S, Toki N, Takeda K, Ohnuma T, Namba Y, Katayama S, et al. Validity of low-intensity continuous renal replacement therapy*. Crit Care Med. 2013;41:2584-91.

238. Schiffl H, Lang SM, Fischer R. Daily hemodialysis and the outcome of acute renal failure. N Engl J Med. 2002;346:305-10.

239. Skofic N, Arnol M, Buturovic-Ponikvar J, Ponikvar R. Intermittent highvolume predilution on-line haemofiltration versus standard intermittent haemodialysis in critically ill patients with acute kidney injury: a prospective randomized study. Nephrol Dial Transplant. 2012;27:4348-56.

240. Faulhaber-Walter R, Hafer C, Jahr N, Vahlbruch J, Hoy L, Haller H, et al. The Hannover Dialysis Outcome study: comparison of standard versus intensified extended dialysis for treatment of patients with acute kidney injury in the intensive care unit. Nephrol Dial Transplant. 2009;24:2179-86.

241. Kaizu K, Inada Y, Kawamura A, Oda S, Hirasawa H. Current status of blood purification in critical care in Japan. Contrib Nephrol. 2010;166:4-10.

242. Davenport A. Continuous renal replacement therapies in patients with acute neurological injury. Semin Dial. 2009;22:165-8.

243. Vanholder R, Van Biesen W, Hoste E, Lameire N. Pro/con debate: continuous versus intermittent dialysis for acute kidney injury: a never-ending story yet approaching the finish? Crit Care. 2011;15:204.

244. Misset B, Timsit JF, Chevret S, Renaud B, Tamion F, Carlet J. A randomized cross-over comparison of the hemodynamic response to intermittent hemodialysis and continuous hemofiltration in ICU patients with acute renal failure. Intensive Care Med. 1996;22:742-6.

245. Mehta RL, McDonald B, Gabbai FB, Pahl M, Pascual MT, Farkas A, et al. A randomized clinical trial of continuous versus intermittent dialysis for acute renal failure. Kidney Int. 2001;60:1154-63.

246. John S, Griesbach D, Baumgartel M, Weihprecht H, Schmieder RE, Geiger H. Effects of continuous haemofiltration vs intermittent haemodialysis on systemic haemodynamics and splanchnic regional perfusion in septic shock patients: a prospective, randomized clinical trial. Nephrol Dial Transplant. 2001;16:320-7

247. Gasparovic V, Filipovic-Grcic I, Merkler M, Pisl Z. Continuous renal replacement therapy (CRRT) or intermittent hemodialysis (IHD) — what is the procedure of choice in critically ill patients? Ren Fail. 2003;25:855-62.

248. Augustine JJ, Sandy D, Seifert TH, Paganini EP. A randomized controlled trial comparing intermittent with continuous dialysis in patients with ARF. Am J Kidney Dis. 2004:44:1000-7.

249. Kielstein JT, Kretschmer U, Ernst T, Hafer C, Bahr MJ, Haller H, et al. Efficacy and cardiovascular tolerability of extended dialysis in critically ill patients: a randomized controlled study. Am J Kidney Dis. 2004;43:342-9.

250. Kumar VA, Yeun JY, Depner TA, Don BR. Extended daily dialysis vs. continuous hemodialysis for ICU patients with acute renal failure: a two-year single center report. Int J Artif Organs. 2004;27:371-9.

251. Uehlinger DE, Jakob SM, Ferrari P, Eichelberger M, Huynh-Do U, Marti HP, et al. Comparison of continuous and intermittent renal replacement therapy for acute renal failure. Nephrol Dial Transplant. 2005;20:1630-7.

252. Vinsonneau C, Camus C, Combes A, Costa de Beauregard MA, Klouche K, Boulain $\mathrm{T}$, et al. Continuous venovenous haemodiafiltration versus intermittent haemodialysis for acute renal failure in patients with multipleorgan dysfunction syndrome: a multicentre randomised trial. Lancet. 2006; 368:379-85.
253. Abe M, Okada K, Suzuki M, Nagura C, Ishihara Y, Fujii Y, et al. Comparison of sustained hemodiafiltration with continuous venovenous hemodiafiltration for the treatment of critically ill patients with acute kidney injury. Artif Organs. 2010;34:331-8.

254. Lins RL, Elseviers MM, Van der Niepen P, Hoste E, Malbrain ML, Damas $P$, et al. Intermittent versus continuous renal replacement therapy for acute kidney injury patients admitted to the intensive care unit: results of a randomized clinical trial. Nephrol Dial Transplant. 2009;24:512-8.

255. Baldwin I, Bellomo R, Naka T, Koch B, Fealy N. A pilot randomized controlled comparison of extended daily dialysis with filtration and continuous venovenous hemofiltration: fluid removal and hemodynamics. Int J Artif Organs. 2007:30:1083-9.

256. Abe M, Maruyama N, Matsumoto S, Okada K, Fujita T, Matsumoto K, et al. Comparison of sustained hemodiafiltration with acetate-free dialysate and continuous venovenous hemodiafiltration for the treatment of critically ill patients with acute kidney injury. Int J Nephrol. 2011;2011:432094.

257. Schwenger V, Weigand MA, Hoffmann O, Dikow R, Kihm LP, Seckinger J, et al. Sustained low efficiency dialysis using a single-pass batch system in acute kidney injury — a randomized interventional trial: the REnal Replacement Therapy Study in Intensive Care Unit PatiEnts. Crit Care. 2012; 16:R140.

258. Schefold JC, von Haehling S, Pschowski R, Bender T, Berkmann C, Briegel S, et al. The effect of continuous versus intermittent renal replacement therapy on the outcome of critically ill patients with acute renal failure (CONVINT): a prospective randomized controlled trial. Crit Care. 2014;18:R11.

259. Kellum JA, Angus DC, Johnson JP, Leblanc M, Griffin M, Ramakrishnan N, et al. Continuous versus intermittent renal replacement therapy: a metaanalysis. Intensive Care Med. 2002;28:29-37.

260. Tonelli M, Manns B, Feller-Kopman D. Acute renal failure in the intensive care unit: a systematic review of the impact of dialytic modality on mortality and renal recovery. Am J Kidney Dis. 2002;40:875-85.

261. Pannu N, Klarenbach S, Wiebe N, Manns B, Tonelli M. Renal replacement therapy in patients with acute renal failure: a systematic review. JAMA. 2008; 299:793-805.

262. Bagshaw SM, Berthiaume LR, Delaney A, Bellomo R. Continuous versus intermittent renal replacement therapy for critically ill patients with acute kidney injury: a meta-analysis. Crit Care Med. 2008;36:610-7.

263. Ghahramani N, Shadrou S, Hollenbeak C. A systematic review of continuous renal replacement therapy and intermittent haemodialysis in management of patients with acute renal failure. Nephrology (Carlton). 2008;13:570-8.

264. Schneider AG, Bellomo R, Bagshaw SM, Glassford NJ, Lo S, Jun M, et al. Choice of renal replacement therapy modality and dialysis dependence after acute kidney injury: a systematic review and meta-analysis. Intensive Care Med. 2013;39:987-97.

265. Rabindranath K, Adams J, Macleod AM, Muirhead N. Intermittent versus continuous renal replacement therapy for acute renal failure in adults. Cochrane Database Syst Rev. 2007:CD003773.

266. Zhang L, Yang J, Eastwood GM, Zhu G, Tanaka A, Bellomo R. Extended daily dialysis versus continuous renal replacement therapy for acute kidney injury: a meta-analysis. Am J Kidney Dis. 2015;66:322-30.

267. Dellinger RP, Levy MM, Rhodes A, Annane D, Gerlach H, Opal SM, et al. Surviving sepsis campaign: international guidelines for management of severe sepsis and septic shock, 2012. Intensive Care Med. 2013;39:165-228.

268. Fieghen HE, Friedrich JO, Burns KE, Nisenbaum R, Adhikari NK, Hladunewich MA, et al. The hemodynamic tolerability and feasibility of sustained low efficiency dialysis in the management of critically ill patients with acute kidney injury. BMC Nephrol. 2010;11:32.

269. Okada H, Suzuki H, Deguchi N, Saruta T. Agranulocytosis in a haemodialysed patient induced by a proteinase inhibitor, nafamostate mesilate. Nephrol Dial Transplant. 1992;7:980.

270. Muto S, Imai M, Asano Y. Mechanisms of hyperkalemia caused by nafamostat mesilate. Gen Pharmacol. 1995;26:1627-32.

271. Maruyama H, Miyakawa Y, Gejyo F, Arakawa M. Anaphylactoid reaction induced by nafamostat mesilate in a hemodialysis patient. Nephron. 1996;74:468-9.

272. Higuchi N, Yamazaki H, Kikuchi H, Gejyo F. Anaphylactoid reaction induced by a protease inhibitor, nafamostat mesilate, following nine administrations in a hemodialysis patient. Nephron. 2000;86:400-1.

273. Lee YK, Lee HW, Choi KH, Kim BS. Ability of nafamostat mesilate to prolong filter patency during continuous renal replacement therapy in patients 
at high risk of bleeding: a randomized controlled study. PLoS One. 2014:9:e108737.

274. Choi JY, Kang YJ, Jang HM, Jung HY, Cho JH, Park SH, et al. Nafamostat mesilate as an anticoagulant during continuous renal replacement therapy in patients with high bleeding risk: a randomized clinical trial. Medicine Baltimore. 2015;94:e2392.

275. Baek NN, Jang HR, Huh W, Kim YG, Kim DJ, Oh HY, et al. The role of nafamostat mesylate in continuous renal replacement therapy among patients at high risk of bleeding. Ren Fail. 2012;34:279-85.

276. Hwang SD, Hyun YK, Moon SJ, Lee SC, Yoon SY. Nafamostat mesilate for anticoagulation in continuous renal replacement therapy. Int J Artif Organs. 2013;36:208-16.

277. Makino S, Egi M, Kita H, Miyatake Y, Kubota K, Mizobuchi S. Comparison of nafamostat mesilate and unfractionated heparin as anticoagulants during continuous renal replacement therapy. Int J Artif Organs. 2016;39:16-21.

278. Monchi M, Berghmans D, Ledoux D, Canivet JL, Dubois B, Damas P. Citrate vs. heparin for anticoagulation in continuous venovenous hemofiltration: a prospective randomized study. Intensive Care Med. 2004;30:260-5.

279. Kutsogiannis DJ, Gibney RT, Stollery D, Gao J. Regional citrate versus systemic heparin anticoagulation for continuous renal replacement in critically ill patients. Kidney Int. 2005;67:2361-7.

280. Betjes MG, van Oosterom D, van Agteren M, van de Wetering J. Regional citrate versus heparin anticoagulation during venovenous hemofiltration in patients at low risk for bleeding: similar hemofilter survival but significantly less bleeding. J Nephrol. 2007;20:602-8.

281. Fealy N, Baldwin I, Johnstone M, Egi M, Bellomo R. A pilot randomized controlled crossover study comparing regional heparinization to regional citrate anticoagulation for continuous venovenous hemofiltration. Int J Artif Organs. 2007;30:301-7.

282. Tiranathanagul K, Jearnsujitwimol O, Susantitaphong P, Kijkriengkraikul N, Leelahavanichkul A, Srisawat N, et al. Regional citrate anticoagulation reduces polymorphonuclear cell degranulation in critically ill patients treated with continuous venovenous hemofiltration. Ther Apher Dial. 2011;15:556-64.

283. Hetzel GR, Schmitz M, Wissing H, Ries W, Schott G, Heering PJ, et al. Regional citrate versus systemic heparin for anticoagulation in critically ill patients on continuous venovenous haemofiltration: a prospective randomized multicentre trial. Nephrol Dial Transplant. 2011;26:232-9.

284. Schilder L, Nurmohamed SA, Bosch FH, Purmer IM, den Boer SS, Kleppe CG, et al. Citrate anticoagulation versus systemic heparinisation in continuous venovenous hemofiltration in critically ill patients with acute kidney injury: a multi-center randomized clinical trial. Crit Care. 2014:18:472.

285. Brain MJ, Roodenburg OS, Adams N, McCracken P, Hockings L, Musgrave S, et al. Randomised trial of software algorithm driven regional citrate anticoagulation versus heparin in continuous renal replacement therapy: the filter life in renal replacement therapy pilot trial. Crit Care Resusc. 2014;16:131-7.

286. Gattas DJ, Rajbhandari D, Bradford C, Buhr H, Lo S, Bellomo R. A randomized controlled trial of regional citrate versus regional heparin anticoagulation for continuous renal replacement therapy in critically ill adults. Crit Care Med. 2015:43:1622-9.

287. Stucker F, Ponte B, Tataw J, Martin PY, Wozniak H, Pugin J, et al. Efficacy and safety of citrate-based anticoagulation compared to heparin in patients with acute kidney injury requiring continuous renal replacement therapy: a randomized controlled trial. Crit Care. 2015;19:91.

288. Reeves JH, Cumming AR, Gallagher L, O'Brien JL, Santamaria JD. A controlled trial of low-molecular-weight heparin (dalteparin) versus unfractionated heparin as anticoagulant during continuous venovenous hemodialysis with filtration. Crit Care Med. 1999;27:2224-8.

289. Joannidis M, Kountchev J, Rauchenzauner M, Schusterschitz N, Ulmer H, Mayr A, et al. Enoxaparin vs. unfractionated heparin for anticoagulation during continuous veno-venous hemofiltration: a randomized controlled crossover study. Intensive Care Med. 2007;33:1571-9.

290. Oudemans-van Straaten HM, Bosman RJ, Koopmans M, van der Voort PH, Wester JP, van der Spoel JI, et al. Citrate anticoagulation for continuous venovenous hemofiltration. Crit Care Med. 2009;37:545-52.

291. Garces EO, Victorino JA, Thome FS, Rohsig LM, Dornelles E, Louzada M, et al. Enoxaparin versus unfractioned heparin as anticoagulant for continuous venovenous hemodialysis: a randomized open-label trial. Ren Fail. 2010;32:320-7.

292. Wu B, Zhang K, Xu B, Ji D, Liu Z, Gong D. Randomized controlled trial to evaluate regional citrate anticoagulation plus low-dose of dalteparin in continuous veno-venous hemofiltration. Blood Purif. 2015;39:306-12.
293. Schiffl H, Lang SM, Konig A, Strasser T, Haider MC, Held E. Biocompatible membranes in acute renal failure: prospective case-controlled study. Lancet. 1994;344:570-2.

294. Jorres A, Gahl GM, Dobis C, Polenakovic MH, Cakalaroski K, Rutkowski B, et al. Haemodialysis-membrane biocompatibility and mortality of patients with dialysis-dependent acute renal failure: a prospective randomised multicentre trial. Int Multicentre Study Group Lancet. 1999;354:1337-41.

295. Gastaldello K, Melot C, Kahn RJ, Vanherweghem JL, Vincent JL, Tielemans C. Comparison of cellulose diacetate and polysulfone membranes in the outcome of acute renal failure. A prospective randomized study. Nephrol Dial Transplant. 2000;15:224-30.

296. Albright RC Jr, Smelser JM, MCCarthy JT, Homburger HA, Bergstralh EJ, Larson TS. Patient survival and renal recovery in acute renal failure: randomized comparison of cellulose acetate and polysulfone membrane dialyzers. Mayo Clin Proc. 2000;75:1141-7.

297. Jones CH, Goutcher E, Newstead CG, Will E, Dean SG, Davison AM. Hemodynamics and survival of patients with acute renal failure treated by continuous dialysis with two synthetic membranes. Artif Organs. 1998;22:638-43.

298. Ponikvar JB, Rus RR, Kenda RB, Bren AF, Ponikvar RR. Low-flux versus highflux synthetic dialysis membrane in acute renal failure: prospective randomized study. Artif Organs. 2001;25:946-50.

299. Haase M, Bellomo R, Baldwin I, Haase-Fielitz A, Fealy N, Davenport P, et al. Hemodialysis membrane with a high-molecular-weight cutoff and cytokine levels in sepsis complicated by acute renal failure: a phase 1 randomized trial. Am J Kidney Dis. 2007;50:296-304.

300. Alonso A, Lau J, Jaber BL. Biocompatible hemodialysis membranes for acute renal failure. Cochrane Database Syst Rev. 2008:CD005283.

301. Hirayama Y, Oda S, Wakabayashi K, Sadahiro T, Nakamura M, Watanabe E, et al. Comparison of interleukin-6 removal properties among hemofilters consisting of varying membrane materials and surface areas: an in vitro study. Blood Purif. 2011;31:18-25.

302. Shiga H, Hirasawa H, Nishida O, Oda S, Nakamura M, Mashiko K, et al. Continuous hemodiafiltration with a cytokine-adsorbing hemofilter in patients with septic shock: a preliminary report. Blood Purif. 2014;38:211-8.

303. Eknoyan G. Emergence of the concept of acute renal failure. Am J Nephrol. 2002;22:225-30

304. Hishida A. Acute renal failure. Nihon Jinzo Gakkai Shi. 2002;44:94-101.

305. Hoste EA, Clermont G, Kersten A, Venkataraman R, Angus DC, De Bacquer D, et al. RIFLE criteria for acute kidney injury are associated with hospital mortality in critically ill patients: a cohort analysis. Crit Care. 2006;10:R73.

306. Uchino S, Bellomo R, Goldsmith D, Bates S, Ronco C. An assessment of the RIFLE criteria for acute renal failure in hospitalized patients. Crit Care Med. 2006:34:1913-7.

307. Ricci Z, Cruz D, Ronco C. The RIFLE criteria and mortality in acute kidney injury: a systematic review. Kidney Int. 2008;73:538-46.

308. Joannidis M, Metnitz B, Bauer P, Schusterschitz N, Moreno R, Druml W, et al. Acute kidney injury in critically ill patients classified by AKIN versus RIFLE using the SAPS 3 database. Intensive Care Med. 2009;35:1692-702.

309. Ali T, Khan I, Simpson W, Prescott G, Townend J, Smith W, et al. Incidence and outcomes in acute kidney injury: a comprehensive population-based study. J Am Soc Nephrol. 2007;18:1292-8.

310. Cruz DN, Bolgan I, Perazella MA, Bonello M, de Cal M, Corradi V, et al. North East Italian Prospective Hospital Renal Outcome Survey on Acute Kidney Injury (NEiPHROS-AKI): targeting the problem with the RIFLE criteria. Clin J Am Soc Nephrol. 2007:2:418-25.

311. Sawhney S, Mitchell M, Marks A, Fluck N, Black C. Long-term prognosis after acute kidney injury (AKI): what is the role of baseline kidney function and recovery? A Sys Rev BMJ Open. 2015;5:e006497.

312. Saratzis A, Harrison S, Barratt J, Sayers RD, Sarafidis PA, Bown MJ. Intervention associated acute kidney injury and long-term cardiovascular outcomes. Am J Nephrol. 2015;42:285-94.

313. Gargiulo G, Sannino A, Capodanno D, Perrino C, Capranzano P, Barbanti M, et al. Impact of postoperative acute kidney injury on clinical outcomes after transcatheter aortic valve implantation: a meta-analysis of 5,971 patients. Catheter Cardiovasc Interv. 2015;86:518-27.

314. Pickering JW, James MT, Palmer SC. Acute kidney injury and prognosis after cardiopulmonary bypass: a meta-analysis of cohort studies. Am J Kidney Dis. 2015;65:283-93.

315. Nisula S, Vaara ST, Kaukonen KM, Reinikainen M, Koivisto SP, Inkinen O, et al. Six-month survival and quality of life of intensive care patients with acute kidney injury. Crit Care. 2013;17:R250. 
316. Hofhuis JG, van Stel HF, Schrijvers AJ, Rommes JH, Spronk PE. The effect of acute kidney injury on long-term health-related quality of life: a prospective follow-up study. Crit Care. 2013;17:R17.

317. Uemura $\mathrm{O}$, Honda M, Matsuyama T, Ishikura K, Hataya H, Yata N, et al. Age, gender, and body length effects on reference serum creatinine levels determined by an enzymatic method in Japanese children: a multicenter study. Clin Exp Nephrol. 2011;15:694-9.

318. Schwartz GJ, Brion LP, Spitzer A. The use of plasma creatinine concentration for estimating glomerular filtration rate in infants, children, and adolescents. Pediatr Clin N Am. 1987;34:571-90.

319. Akcan-Arikan A, Zappitelli M, Loftis LL, Washburn KK, Jefferson LS, Goldstein SL. Modified RIFLE criteria in critically ill children with acute kidney injury. Kidney Int. 2007;71:1028-35.

320. Uemura O, Honda M, Matsuyama T, Ishikura K, Hataya H, Nagai T, et al. Is the new Schwartz equation derived from serum creatinine and body length suitable for evaluation of renal function in Japanese children? Eur J Pediatr. 2012:171:1401-4.

321. Uemura O, Nagai T, Ishikura K, Ito S, Hataya H, Gotoh Y, et al. Creatininebased equation to estimate the glomerular filtration rate in Japanese children and adolescents with chronic kidney disease. Clin Exp Nephrol. 2014;18:626-33.

322. Sutherland SM, Byrnes JJ, Kothari M, Longhurst CA, Dutta S, Garcia P, et al. AKI in hospitalized children: comparing the pRIFLE, AKIN, and KDIGO definitions. Clin J Am Soc Nephrol. 2015;10:554-61.

323. Selewski DT, Cornell TT, Heung M, Troost JP, Ehrmann BJ, Lombel RM, et al. Validation of the KDIGO acute kidney injury criteria in a pediatric critical care population. Intensive Care Med. 2014;40:1481-8.

324. Jetton JG, Askenazi DJ. Acute kidney injury in the neonate. Clin Perinatol. 2014:41:487-502.

325. Selewski DT, Charlton JR, Jetton JG, Guillet R, Mhanna MJ, Askenazi DJ, et al. Neonatal acute kidney injury. Pediatrics. 2015;136:e463-73.

326. Guignard JP, Drukker A. Why do newborn infants have a high plasma creatinine? Pediatrics. 1999;103:e49.

327. Boer DP, de Rijke YB, Hop WC, Cransberg K, Dorresteijn EM. Reference values for serum creatinine in children younger than 1 year of age. Pediatr Nephrol. 2010;25:2107-13.

328. Bateman DA, Thomas W, Parravicini E, Polesana E, Locatelli C, Lorenz JM. Serum creatinine concentration in very-low-birth-weight infants from birth to 34-36 wk postmenstrual age. Pediatr Res. 2015;77:696-702.

329. Koralkar R, Ambalavanan N, Levitan EB, McGwin G, Goldstein S, Askenazi D. Acute kidney injury reduces survival in very low birth weight infants. Pediatr Res. 2011;69:354-8.

330. Carmody JB, Swanson JR, Rhone ET, Charlton JR. Recognition and reporting of AKI in very low birth weight infants. Clin J Am Soc Nephrol. 2014;9:2036-43.

331. Rhone ET, Carmody JB, Swanson JR, Charlton JR. Nephrotoxic medication exposure in very low birth weight infants. J Matern Fetal Neonatal Med. 2014:27:1485-90.

332. Sarkar S, Askenazi DJ, Jordan BK, Bhagat I, Bapuraj JR, Dechert RE, et al. Relationship between acute kidney injury and brain MRI findings in asphyxiated newborns after therapeutic hypothermia. Pediatr Res. 2014;75:431-5.

333. Mishra J, Dent C, Tarabishi R, Mitsnefes MM, Ma Q, Kelly C, et al. Neutrophi gelatinase-associated lipocalin (NGAL) as a biomarker for acute renal injury after cardiac surgery. Lancet. 2005;365:1231-8.

334. Parikh CR, Devarajan P, Zappitelli M, Sint K, Thiessen-Philbrook H, Li S, et al Postoperative biomarkers predict acute kidney injury and poor outcomes after pediatric cardiac surgery. J Am Soc Nephrol. 2011;22:1737-47.

335. Zappitelli M, Washburn KK, Arikan AA, Loftis L, Ma Q, Devarajan P, et al. Urine neutrophil gelatinase-associated lipocalin is an early marker of acute kidney injury in critically ill children: a prospective cohort study. Crit Care. 2007;11:R84

336. Bennett M, Dent CL, Ma Q, Dastrala S, Grenier F, Workman R, et al. Urine NGAL predicts severity of acute kidney injury after cardiac surgery: a prospective study. Clin J Am Soc Nephrol. 2008:3:665-73.

337. Dent $C L$, Ma Q, Dastrala S, Bennett M, Mitsnefes MM, Barasch J, et al. Plasma neutrophil gelatinase-associated lipocalin predicts acute kidney injury, morbidity and mortality after pediatric cardiac surgery: a prospective uncontrolled cohort study. Crit Care. 2007;11:R127.

338. Krawczeski CD, Vandevoorde RG, Kathman T, Bennett MR, Woo JG, Wang Y, et al. Serum cystatin $C$ is an early predictive biomarker of acute kidney injury after pediatric cardiopulmonary bypass. Clin J Am Soc Nephrol. 2010;5:1552-7.
339. Zappitelli M, Krawczeski CD, Devarajan P, Wang Z, Sint K, Thiessen-Philbrook $\mathrm{H}$, et al. Early postoperative serum cystatin $\mathrm{C}$ predicts severe acute kidney injury following pediatric cardiac surgery. Kidney Int. 2011;80:655-62.

340. Parikh CR, Mishra J, Thiessen-Philbrook H, Dursun B, Ma Q, Kelly C, et al. Urinary IL-18 is an early predictive biomarker of acute kidney injury after cardiac surgery. Kidney Int. 2006;70:199-203.

341. Liu Y, Guo W, Zhang J, Xu C, Yu S, Mao Z, et al. Urinary interleukin 18 for detection of acute kidney injury: a meta-analysis. Am J Kidney Dis. 2013;62: 1058-67.

342. Portilla D, Dent C, Sugaya T, Nagothu KK, Kundi I, Moore P, et al. Liver fatty acid-binding protein as a biomarker of acute kidney injury after cardiac surgery. Kidney Int. 2008;73:465-72.

343. Han WK, Waikar SS, Johnson A, Betensky RA, Dent CL, Devarajan P, et al. Urinary biomarkers in the early diagnosis of acute kidney injury. Kidney Int. 2008;73:863-9.

344. Zappitelli M, Coca SG, Garg AX, Krawczeski CD, Thiessen Heather P, Sint K, et al. The association of albumin/creatinine ratio with postoperative AKI in children undergoing cardiac surgery. Clin J Am Soc Nephrol. 2012;7:1761-9.

345. Krawczeski CD, Goldstein SL, Woo JG, Wang Y, Piyaphanee N, Ma Q, et al. Temporal relationship and predictive value of urinary acute kidney injury biomarkers after pediatric cardiopulmonary bypass. J Am Coll Cardiol. 2011; 58:2301-9.

346. Basu RK, Wong HR, Krawczeski CD, Wheeler DS, Manning PB, Chawla LS, et al. Combining functional and tubular damage biomarkers improves diagnostic precision for acute kidney injury after cardiac surgery. J Am Coll Cardiol. 2014;64:2753-62.

347. Goldstein SL, Currier H, Graf C, Cosio CC, Brewer ED, Sachdeva R. Outcome in children receiving continuous venovenous hemofiltration. Pediatrics. 2001;107:1309-12.

348. Goldstein SL, Somers MJ, Baum MA, Symons JM, Brophy PD, Blowey D, et al. Pediatric patients with multi-organ dysfunction syndrome receiving continuous renal replacement therapy. Kidney Int. 2005;67:653-8.

349. Sutherland SM, Zappitelli M, Alexander SR, Chua AN, Brophy PD, Bunchman $\mathrm{TE}$, et al. Fluid overload and mortality in children receiving continuous renal replacement therapy: the prospective pediatric continuous renal replacement therapy registry. Am J Kidney Dis. 2010;55:316-25.

350. Modem V, Thompson M, Gollhofer D, Dhar AV, Quigley R. Timing of continuous renal replacement therapy and mortality in critically ill children*. Crit Care Med. 2014;42:943-53.

351. Foland JA, Fortenberry JD, Warshaw BL, Pettignano R, Merritt RK, Heard ML, et al. Fluid overload before continuous hemofiltration and survival in critically ill children: a retrospective analysis. Crit Care Med. 2004;32:1771-6.

352. Gillespie RS, Seidel K, Symons JM. Effect of fluid overload and dose of replacement fluid on survival in hemofiltration. Pediatr Nephrol. 2004; 19:1394-9.

353. Hayes LW, Oster RA, Tofil NM, Tolwani AJ. Outcomes of critically ill children requiring continuous renal replacement therapy. J Crit Care. 2009;24:394-400.

354. Michael M, Kuehnle I, Goldstein SL. Fluid overload and acute renal failure in pediatric stem cell transplant patients. Pediatr Nephrol. 2004;19:91-5.

355. Selewski DT, Cornell TT, Blatt NB, Han YY, Mottes T, Kommareddi M, et al. Fluid overload and fluid removal in pediatric patients on extracorporeal membrane oxygenation requiring continuous renal replacement therapy. Crit Care Med. 2012:40:2694-9.

356. Sanchez-de-Toledo J, Perez-Ortiz A, Gil L, Baust T, Lines-Palazon M, PerezHoyos S, et al. Early initiation of renal replacement therapy in pediatric heart surgery is associated with lower mortality. Pediatr Cardiol. 2015;37:623-8.

357. Selewski DT, Cornell TT, Lombel RM, Blatt NB, Han YY, Mottes T, et al. Weight-based determination of fluid overload status and mortality in pediatric intensive care unit patients requiring continuous renal replacement therapy. Intensive Care Med. 2011;37:1166-73.

358. Kara OD, Dincel N, Kaplan Bulut I, Yilmaz E, Ozdemir K, Gozuoglu G, et al. Success of continuous veno-venous hemodiafiltration treatment in children monitored in the intensive care units. Ren Fail. 2014;36:1411-5.

359. de Galasso L, Emma F, Picca S, Di Nardo M, Rossetti E, Guzzo I. Continuous renal replacement therapy in children: fluid overload does not always predict mortality. Pediatr Nephrol. 2016;31:651-9.

360. Duzova A, Bakkaloglu A, Kalyoncu M, Poyrazoglu H, Delibas A, Ozkaya O, et al. Etiology and outcome of acute kidney injury in children. Pediatr Nephrol. 2010;25:1453-61.

361. Agras PI, Tarcan A, Baskin E, Cengiz N, Gurakan B, Saatci U. Acute rena failure in the neonatal period. Ren Fail. 2004;26:305-9. 
362. Bolat F, Comert S, Bolat G, Kucuk O, Can E, Bulbul A, et al. Acute kidney injury in a single neonatal intensive care unit in Turkey. World J Pediatr. 2013;9:323-9.

363. Bezerra $C T$, Vaz Cunha LC, Liborio AB. Defining reduced urine output in neonatal ICU: importance for mortality and acute kidney injury classification. Nephrol Dial Transplant. 2013;28:901-9.

364. Hui-Stickle S, Brewer ED, Goldstein SL. Pediatric ARF epidemiology at a tertiary care center from 1999 to 2001. Am J Kidney Dis. 2005;45:96-101.

365. Askenazi DJ, Ambalavanan N, Hamilton K, Cutter G, Laney D, Kaslow R, et al. Acute kidney injury and renal replacement therapy independently predict mortality in neonatal and pediatric noncardiac patients on extracorporeal membrane oxygenation. Pediatr Crit Care Med. 2011;12:e1-6.

366. Alabbas A, Campbell A, Skippen P, Human D, Matsell D, Mammen C. Epidemiology of cardiac surgery-associated acute kidney injury in neonates: a retrospective study. Pediatr Nephrol. 2013;28:1127-34.

367. Selewski DT, Jordan BK, Askenazi DJ, Dechert RE, Sarkar S. Acute kidney injury in asphyxiated newborns treated with therapeutic hypothermia. J Pediatr. 2013;162:725-9. e1

368. Sutherland SM, Ji J, Sheikhi FH, Widen E, Tian L, Alexander SR, et al. AKI in hospitalized children: epidemiology and clinical associations in a national cohort. Clin J Am Soc Nephrol. 2013:8:1661-9.

369. Cataldi L, Leone R, Moretti U, De Mitri B, Fanos V, Ruggeri L, et al. Potential risk factors for the development of acute renal failure in preterm newborn infants: a case-control study. Arch Dis Child Fetal Neonatal Ed. 2005;90:F514-9.

370. Mortazavi F, Hosseinpour Sakha S, Nejati N. Acute kidney failure in neonatal period. Iran J Kidney Dis. 2009;3:136-40.

371. Viswanathan S, Manyam B, Azhibekov T, Mhanna MJ. Risk factors associated with acute kidney injury in extremely low birth weight (ELBW) infants. Pediatr Nephrol. 2012;27:303-11.

372. Askenazi DJ, Griffin R, McGwin G, Carlo W, Ambalavanan N. Acute kidney injury is independently associated with mortality in very low birthweight infants: a matched case-control analysis. Pediatr Nephrol. 2009;24:991-7.

373. Walker MW, Clark RH, Spitzer AR. Elevation in plasma creatinine and renal failure in premature neonates without major anomalies: terminology, occurrence and factors associated with increased risk. J Perinatol. 2011; 31:199-205.

374. Morgan CJ, Zappitelli M, Robertson CM, Alton GY, Sauve RS, Joffe AR, et al. Risk factors for and outcomes of acute kidney injury in neonates undergoing complex cardiac surgery. J Pediatr. 2013;162:120-7. e1

375. Gupta BD, Sharma P, Bagla J, Parakh M, Soni JP. Renal failure in asphyxiated neonates. Indian Pediatr. 2005;42:928-34.

376. Perlman JM, Tack ED. Renal injury in the asphyxiated newborn infant: relationship to neurologic outcome. J Pediatr. 1988;113:875-9.

377. Morelli S, Ricci Z, Di Chiara L, Stazi GV, Polito A, Vitale V, et al. Renal replacement therapy in neonates with congenital heart disease. Contrib Nephrol. 2007;156:428-33.

378. Blinder JJ, Goldstein SL, Lee W, Baycroft A, Fraser CD, Nelson D, et al. Congenital heart surgery in infants: effects of acute kidney injury on outcomes. J Thorac Cardiovasc Surg. 2012;143:368-74.

379. Zappitelli M, Bernier PL, Saczkowski RS, Tchervenkov Cl, Gottesman R, Dancea $A$, et al. A small post-operative rise in serum creatinine predicts acute kidney injury in children undergoing cardiac surgery. Kidney Int. 2009; 76:885-92.

380. Mathur NB, Agarwal HS, Maria A. Acute renal failure in neonatal sepsis. Indian J Pediatr. 2006;73:499-502.

381. Bunchman TE, McBryde KD, Mottes TE, Gardner JJ, Maxvold NJ, Brophy PD. Pediatric acute renal failure: outcome by modality and disease. Pediatr Nephrol. 2001;16:1067-71.

382. Maxvold NJ, Smoyer WE, Gardner JJ, Bunchman TE. Management of acute renal failure in the pediatric patient: hemofiltration versus hemodialysis. Am J Kidney Dis. 1997;30:584-8.

383. Vachvanichsanong $P$, Dissaneewate $P$, Lim A, McNeil E. Childhood acute renal failure: 22-year experience in a university hospital in southern Thailand. Pediatrics. 2006:118:e786-91.

384. Bailey D, Phan V, Litalien C, Ducruet T, Merouani A, Lacroix J, et al. Risk factors of acute renal failure in critically ill children: a prospective descriptive epidemiological study. Pediatr Crit Care Med. 2007;8:29-35.

385. Hackbarth RM, Eding D, Gianoli Smith C, Koch A, Sanfilippo DJ, Bunchman TE. Zero balance ultrafiltration (Z-BUF) in blood-primed CRRT circuits achieves electrolyte and acid-base homeostasis prior to patient connection. Pediatr Nephrol. 2005;20:1328-33.

386. Symons JM, Chua AN, Somers MJ, Baum MA, Bunchman TE, Benfield MR, et al. Demographic characteristics of pediatric continuous renal replacement therapy: a report of the prospective pediatric continuous renal replacement therapy registry. Clin J Am Soc Nephrol. 2007;2:732-8.

387. Askenazi DJ, Goldstein SL, Koralkar R, Fortenberry J, Baum M, Hackbarth R, et al. Continuous renal replacement therapy for children $\leq 10 \mathrm{~kg}$ : a report from the prospective pediatric continuous renal replacement therapy registry. J Pediatr. 2013;162:587-92. e3

388. Yu JE, Park MS, Pai KS. Acute peritoneal dialysis in very low birth weight neonates using a vascular catheter. Pediatr Nephrol. 2010;25:367-71.

389. Auron A, Warady BA, Simon S, Blowey DL, Srivastava T, Musharaf G, et al. Use of the multipurpose drainage catheter for the provision of acute peritoneal dialysis in infants and children. Am J Kidney Dis. 2007:49:650-5.

390. Warady BA, Bunchman T. Dialysis therapy for children with acute renal failure: survey results. Pediatr Nephrol. 2000;15:11-3.

391. Symons JM, Brophy PD, Gregory MJ, McAfee N, Somers MJ, Bunchman TE, et al. Continuous renal replacement therapy in children up to $10 \mathrm{~kg}$. Am J Kidney Dis. 2003;41:984-9.

392. Ronco C, Garzotto F, Brendolan A, Zanella M, Bellettato M, Vedovato S, et al. Continuous renal replacement therapy in neonates and small infants: development and first-in-human use of a miniaturised machine (CARPEDIEM). Lancet. 2014;383:1807-13.

393. Coulthard MG, Crosier J, Griffiths C, Smith J, Drinnan M, Whitaker M, et al. Haemodialysing babies weighing $<8 \mathrm{~kg}$ with the Newcastle infant dialysis and ultrafiltration system (Nidus): comparison with peritoneal and conventional haemodialysis. Pediatr Nephrol. 2014;29:1873-81.

394. Ronco C, Bellomo R, Brendolan A, Pinna V, La Greca G. Brain density changes during renal replacement in critically ill patients with acute renal failure. Continuous hemofiltration versus intermittent hemodialysis. J Nephrol. 1999;12:173-8.

395. Yavascan O, Kara OD, Anil M, Bal A, Pehlivan O, Aksu N. Chronic peritoneal dialysis treatment in a pediatric patient with Down syndrome. Perit Dial Int 2008;28:558-9.

396. Kupferman JC, Stewart CL, Kaskel FJ, Katz SP, Fine RN. Chronic peritoneal dialysis in a child with Down syndrome. Pediatr Nephrol. 1994;8:644-5.

397. Webb N, Hebert D, Arbus G. Renal replacement therapy in Down's syndrome. Pediatr Nephrol. 1993;7:771.

398. Hsu CY, McCulloch CE, Fan D, Ordonez JD, Chertow GM, Go AS. Communitybased incidence of acute renal failure. Kidney Int. 2007;72:208-12.

399. Zhang L, Fu P, Wang L, Cai G, Chen D, Guo D, et al. The clinical features and outcome of crush patients with acute kidney injury after the Wenchuan earthquake: differences between elderly and younger adults. Injury. 2012;43:1470-5.

400. Rule AD, Amer H, Cornell LD, Taler SJ, Cosio FG, Kremers WK, et al. The association between age and nephrosclerosis on renal biopsy among healthy adults. Ann Intern Med. 2010;152:561-7.

401. Grams ME, Sang Y, Ballew SH, Gansevoort RT, Kimm H, Kovesdy CP, et al. A meta-analysis of the association of estimated GFR, albuminuria, age, race, and sex with acute kidney injury. Am J Kidney Dis. 2015;66:591-601.

402. Samiy AH. Renal disease in the elderly. Med Clin North Am. 1983;67:463-80.

403. Chaumont M, Pourcelet A, van Nuffelen M, Racape J, Leeman M, Hougardy JM. Acute kidney injury in elderly patients with chronic kidney disease: do angiotensin-converting enzyme inhibitors carry a risk? J Clin Hypertens (Greenwich). 2016:18:514-21.

404. Mehran R, Aymong ED, Nikolsky E, Lasic Z, lakovou I, Fahy M, et al. A simple risk score for prediction of contrast-induced nephropathy after percutaneous coronary intervention: development and initial validation. J Am Coll Cardiol. 2004;44:1393-9.

405. Kane-Gill SL, Sileanu FE, Murugan R, Trietley GS, Handler SM, Kellum JA. Risk factors for acute kidney injury in older adults with critical illness: a retrospective cohort study. Am J Kidney Dis. 2015;65:860-9.

406. Skarupskiene I, Balciuviene V, Ziginskiene E, Kuzminskis V, Vaiciuniene R, Bumblyte IA. Changes of etiology, incidence and outcomes of severe acute kidney injury during a 12-year period (2001-2012) in large university hospital. Nephrol Ther. 2016;12:448-53.

407. Bagshaw SM, Webb SA, Delaney A, George C, Pilcher D, Hart GK, et al. Very old patients admitted to intensive care in Australia and New Zealand: a multi-centre cohort analysis. Crit Care. 2009;13:R45.

408. Carlson N, Hommel K, Olesen JB, Soja AM, Vilsboll T, Kamper AL, et al. Dialysis-requiring acute kidney injury in Denmark 2000-2012: time trends of 
incidence and prevalence of risk factors — a nationwide study. PLoS One. 2016;11:0148809.

409. Kayatas K, Sahin G, Tepe M, Kaya ZE, Apaydin S, Demirtunc R. Acute kidney injury in the elderly hospitalized patients. Ren Fail. 2014;36:1273-7.

410. Palevsky PM. Renal replacement therapy in acute kidney injury. Adv Chronic Kidney Dis. 2013;20:76-84.

411. Liu S, Cheng QL, Zhang XY, Ma Q, Liu YL, Pan R, et al. Application of continuous renal replacement therapy for acute kidney injury in elderly patients. Int J Clin Exp Med. 2015;8:9973-8.

412. Chima-Melton C, Murphy TE, Araujo KL, Pisani MA. The impact of race on intensity of care provided to older adults in the medical intensive care unit. J Racial Ethn Health Disparities. 2016;3:365-72.

413. Wilson FP, Yang W, Machado CA, Mariani LH, Borovskiy Y, Berns JS, et al. Dialysis versus nondialysis in patients with AKI: a propensity-matched cohort study. Clin J Am Soc Nephrol. 2014;9:673-81.

414. Lin YT, Wu PH, Kuo MC, Lin MY, Lee TC, Chiu YW, et al. High cost and low survival rate in high comorbidity incident elderly hemodialysis patients. PLoS One. 2013;8:e75318.

415. Abdel-Kader K, Palevsky PM. Acute kidney injury in the elderly. Clin Geriatr Med. 2009;25:331-58.

416. Coca SG. Acute kidney injury in elderly persons. Am J Kidney Dis. 2010;56: $122-31$.

417. Ishani A, Xue JL, Himmelfarb J, Eggers PW, Kimmel PL, Molitoris BA, et al. Acute kidney injury increases risk of ESRD among elderly. J Am Soc Nephrol. 2009;20:223-8

418. Newsome BB, Warnock DG, McClellan WM, Herzog CA, Kiefe Cl, Eggers PW, et al. Long-term risk of mortality and end-stage renal disease among the elderly after small increases in serum creatinine level during hospitalization for acute myocardial infarction. Arch Intern Med. 2008;168:609-16.

419. Thakar CV, Christianson A, Himmelfarb J, Leonard AC. Acute kidney injury episodes and chronic kidney disease risk in diabetes mellitus. Clin J Am Soc Nephrol. 2011;6:2567-72.

420. Wong SP, Kreuter W, O'Hare AM. Healthcare intensity at initiation of chronic dialysis among older adults. J Am Soc Nephrol. 2014;25:143-9.

421. Crews DC, Scialla JJ, Liu J, Guo H, Bandeen-Roche K, Ephraim PL, et al. Predialysis health, dialysis timing, and outcomes among older United States adults. J Am Soc Nephrol. 2014;25:370-9.

422. Testani JM, Chen J, McCauley BD, Kimmel SE, Shannon RP. Potential effects of aggressive decongestion during the treatment of decompensated heart failure on renal function and survival. Circulation. 2010;122:265-72.

423. Pfister R, Muller-Ehmsen J, Hagemeister J, Hellmich M, Erdmann E, Schneider CA. NT-pro-BNP predicts worsening renal function in patients with chronic systolic heart failure. Intern Med J. 2011;41:467-72.

Ready to submit your research? Choose BMC and benefit from:

- fast, convenient online submission

- thorough peer review by experienced researchers in your field

- rapid publication on acceptance

- support for research data, including large and complex data types

- gold Open Access which fosters wider collaboration and increased citations

- maximum visibility for your research: over $100 \mathrm{M}$ website views per year

At BMC, research is always in progress.

Learn more biomedcentral.com/submissions 